\title{
Present Worth Factors for Life-Cycle Cost Studies in the Department of Defense (1996)
}

Data for DoD compliance with the Federal Methodology for Life-Cycle Cost Analysis, Title 10, CFR, Part 436, Subpart A, and OMB Circular A-94

Stephen R. Petersen

Office of Applied Economics

Building and Fire Research Laboratory

Gaithersburg, Maryland 20899

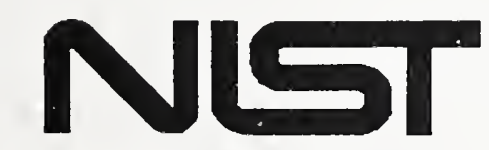

QC

Trases States Department of Commerce

100 ogy Administration Institute of Standards and Technology
Sponsored by:

The Federal Energy Management Program

U.S. Department of Energy

Washington, DC 20585

.456

mo. 4942

1995 



\section{Present Worth Factors for Life-Cycle Cost Studies in the Department of Defense (1996)}

Data for DoD compliance with the Federal Methodology for Life-Cycle Cost Analysis, Title 10, CFR, Part

436, Subpart A, and OMB Circular A-94

Stephen R. Petersen

Office of Applied Economics

October 1995

Building and Fire Research Laboratory

National Institute of Standards and Technology

Gaithersburg, Maryland 20899

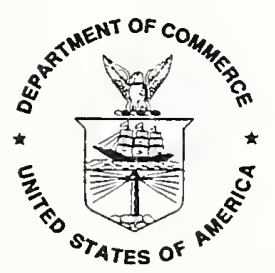

U.S. Department of Commerce

Ronald H. Brown, Secretary

Technology Administration

Mary L. Good, Under Secretary for Technology

National Institute of Standards and Technology

Arati Prabhakar, Director
Sponsored by:

The Federal Energy Management Program

U.S. Department of Energy

Washington, DC 20585 



\begin{abstract}
This document provides 47 tables of present worth factors to be used in computing the present worth of future costs (or cost reductions) in economic analyses of design decisions for projects in the DoD Military Construction Program. These factors are especially useful for the life-cycle cost analysis of investments in buildings or building systems which are intended to reduce future operating, maintenance, repair, replacement, and energy costs over the life of the facility. The tables include present worth factors for both one-time costs and annually recurring costs, based on the FEMP discount rate of $4.1 \%$ (FY 1996) for energy-related studies and on the OMB discount rate of $4.6 \%$ and $4.9 \%$ for short-term and long-term non-energy studies, respectively. Forecasts of future energy prices used in the calculation of present worth factors for energy costs were provided by the Energy Information Administration.
\end{abstract}




\section{PREFACE}

On March 18, 1991, and again in March 1994, the Army, Navy, and Air Force signed a Memorandum of Agreement (MOA) on Criteria/Standards for Economic Analysis/Life-Cycle Costing for MILCON Design. The stated purpose of these MOAs is to establish criteria and standards for performing economic analyses (EAs) and life-cycle cost (LCC) studies in support of design decisions for projects in the Military Construction (MILCON) Program; i.e., to support the selection from various alternatives of components/systems being considered as elements in facilities design. Since 1991 the criteria/standards package represented by the provisions of the MOA has been adopted and specified for use in conjunction with several other Department of Defense (DoD) applications. For example, the Office of the Secretary of Defense has specified that EAs conducted in support of project-justification decisions for ECIP (Energy Conservation Investment Program) projects be based on this same criteria/standards package.

The criteria and standards in the MOA are responsive to, and consistent with, the requirements of 10 CFR 436 governing the economic analysis of investments in energy conservation and renewable energy resources for Federal facilities. However, the governing order for non-energy-related investments, OMB Circular A-94, was significantly revised in October 1992. Circular A-94 no longer specifies a single discount rate for economic analysis of Federal investments. Instead, Circular A-94 specifies two basic types of discount rates: (1) a discount rate for cost-effectiveness, lease-purchase, and related analyses; and (2) a discount rate for public investment and regulatory analyses. Only discount rates for the first type of analyses are included in this NIST report, since its primary purpose is to support cost-effectiveness studies related to the design and operation of DoD facilities.

OMB discount rates for cost-effectiveness and lease-purchase studies are based on interest rates on Treasury Notes and Bonds with maturities ranging from 3 to 30 years. Currently (as of January 1995) five maturities have been specifically identified by OMB, and are shown here with the corresponding real interest rate to be used as the discount rate for studies subject to Circular A-94:

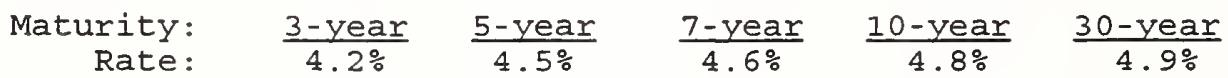

For total study periods corresponding to these five maturities, Circular A-94 requires that the discount rates correspond to the rates shown. The total study period is the time between the Beneficial Occupancy Date and the end of study (the service period), plus the time between the Date of Study and the Beneficial Occupancy Date (the planning and construction period). For total study periods different from the five maturities shown, Circular A-94 does not specify the discount rate to be used. Although Circular A-94 allows for interpolation between the rates shown, such an approach is not practical for the simplified tabular approach used in this document. Accordingly, the enclosed tables have been developed for use primarily for long-term studies, defined here as those with total study periods exceeding 10 years. These data are based on a discount rate of $4.9 \%$, corresponding to OMB's 30-year rate. 
The tables have also been designed for use in support of short-term studies, defined here as those with total study periods of 10 years or less. For these types of studies, the present worth factors are based on a discount rate of $4.6 \%$, corresponding to OMB's 7-year rate. Accordingly, for short-term studies with total study periods not close to seven years, the discount factors provided in the tables should be considered approximations only.

When more precise results are required, the present worth factors should be determined using a computer program specifically approved for DoD-construction design applications. The Life Cycle Cost in Design (LCCID) program, developed by the U.S. Army Corps of Engineers' Construction Engineering Research Laboratory (CERL) specifically for DoD-construction design applications, is recommended. The NIST Building Life Cycle Cost (BLCC) or DISCOUNT programs, developed for more general applications, are also acceptable. The NIST BLCC program (versions 4.3-96 or later) now provides ECIP reporting capabilities. Whichever computer program is used, it must be the most recent version of that program, which incorporates the latest DOE-projected energy price escalation rates and the discount rates for energy and non-energy studies.

The tables presented in this document are designed to be used in support of EAs/LCC studies conducted in accordance with the provisions of the MOA. These tables are considered to be valid and appropriate for all analyses/studies initiated during Fiscal Year 1996, and are authorized for use throughout that period. The present worth factors presented in the annually recurring cost tables are based on an assumed Date of Study (DOS) of April 1996. However, these factors should be sufficiently accurate for use at any time during that fiscal year.

The present worth factors in this report are consistent with those in NISTIR 85-3273-10, Energy Price Indices and Discount Factors for Life-Cycle Cost Analysis 1996, the Annual Supplement to NIST Handbook 135, Life-Cycle Costing Manual for the Federal Energy Management Program. However, the present worth factors for DoD studies are more specific as to the Date of Study and the Beneficial Occupancy Date than those in NISTIR 85-3273-10. The DoD factors for annually recurring costs assume that the Date of Study is April 1996 and that the Beneficial Occupancy Date occurs in October of the current year or in October of a future year (up to 2005). The DoD tables for annually recurring costs are based on a mid-year discounting convention for all annually-recurring costs, while the corresponding tables in NISTIR 85-3273-10 are based on an end-of-year discounting convention.

The same forecast of regional energy prices provided by the U.S. Department of Energy's Energy Information Administration (EIA) for NISTIR 85-3273-10 were used in computing the energy-typespecific tables in this report. However, with one exception, these tables are based on forecasts of energy prices for the industrial sector only, while the tables in NISTIR 85-3273-10 are based on residential, commercial, and industrial forecasts. Price forecasts for liquefied petroleum gas (LPG) in the residential sector were used for the DoD tables because industrial LPG price forecasts were not available from EIA.

For LCC analysis of Federal projects outside of the Department of Defense, especially those projects related to energy conservation and renewable energy resources, the present worth factors from NISTIR 85-3273-10 should be used. Note that the terms "present worth factor" (preferred by DoD) and "present value factor" (used in NISTIR 85-3273-10 and NIST Handbook 135) have identical meanings. 
Both the CERL LCCID Program and the NIST Building Life-Cycle Program (BLCC), version 4.0 or later, can be used for the economic evaluation of MILCON design projects, consistent with the Tri-Service MOAs of 1991 and 1994. Both use the DOE projections of energy price increases (which are on the LCCID and BLCC disks) to calculate the present value of future energy costs. Both provide a capability for preparing ECIP reports. In addition, the NIST ERATES program ( $A$ Computer Program for Calculating Time-of-Use, Block, and Demand Charges for Electricity Usage, NISTIR 5186, July 1993) can be used to incorporate complex electricity rate schedules into a BLCC analysis of building energy use. A new NIST program, EMISS (A Program for Estimating Local Air Polution Emission Factors Related to Energy Use in Buildings, NISTIR 5704, October 1995) enables BLCC to estimate reductions in local air polution emissions attributable to energy conservation projects.

The annual update to Handbook 135, as well as NIST Handbook 135, BLCC, ERATES, and EMISS can be obtained from:

Advanced Sciences, Inc.

1525 Wilson Blvd.

Suite 1200

Arlington, VA 22209

(703) $243-4900$, fax (703) 524-5237 


\section{ACKNOWLEDGMENTS}

The author wishes to thank Larry Schindler of the U.S. Army Corps of Engineers Headquarters Office for providing the requirements of the tables and for his careful review of this manuscript. The author also wishes to thank Linda Lawrie of the U.S. Army Corps of Engineers' Construction Engineering Research Laboratory for providing the basic format of the tables as used in prior years, upon which these tables are based. Appreciation is extended to Mr. Art Andersen, Director of the Energy Demand and Integration Division, and Susan Shaw, Chief, and Paul Condis of the National Energy Modeling System Branch, DOE Energy Information Administration, for providing the energy price projections upon which this report is based. 


\section{CONTENTS}

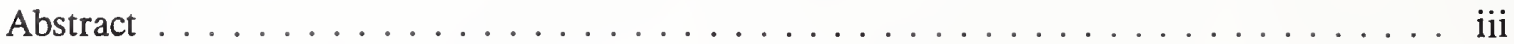

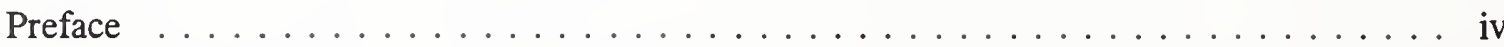

Acknowledgments ................................. . . . . .

Part I. Tables of Present Worth Factors for Energy Studies . . . . . . . . . . . 1

Table Title

E-1. One-Time Costs, Zero Differential Escalation . . . . . . . . . . 2

E-2. Annually Recurring Non-Energy Costs, Zero Differential Escalation ..... 3

E-3-EL-1. Electricity, Region $1 \ldots \ldots \ldots \ldots \ldots \ldots \ldots$

E-3-DO-1. Distillate Oil, Region $1 \ldots \ldots \ldots \ldots \ldots \ldots$

E-3-RO-1. Residual Oil, Region $1 \ldots \ldots \ldots \ldots \ldots$

E-3-NG-1. Natural Gas, Region $1 \ldots \ldots \ldots \ldots \ldots \ldots \ldots$

E-3-SC-1. Steam Coal, Region $1 \ldots \ldots \ldots \ldots \ldots$

E-3-LP-1. Liquefied Petroleum Gas (LPG), Region $1 \ldots \ldots \ldots \ldots$

E-3-EL-2. Electricity, Region $2 \ldots \ldots \ldots \ldots \ldots \ldots$

E-3-DO-2. Distillate Oil, Region $2 \ldots \ldots \ldots \ldots \ldots \ldots \ldots \ldots \ldots$

E-3-RO-2. Residual Oil, Region $2 \ldots \ldots \ldots \ldots \ldots \ldots \ldots \ldots \ldots$

E-3-NG-2. Natural Gas, Region $2 \ldots \ldots \ldots \ldots \ldots \ldots \ldots$

E-3-SC-2. Steam Coal, Region $2 \ldots \ldots \ldots \ldots \ldots \ldots \ldots \ldots$

E-3-LP-2. Liquefied Petroleum Gas (LPG), Region $2 \ldots \ldots \ldots \ldots$

E-3-EL-3. Electricity, Region $3 \ldots \ldots \ldots \ldots \ldots \ldots \ldots$

E-3-DO-3. Distillate Oil, Region $3 \ldots \ldots \ldots \ldots \ldots \ldots \ldots \ldots$

E-3-RO-3. Residual Oil, Region $3 \ldots \ldots \ldots \ldots \ldots$

E-3-NG-3. Natural Gas, Region $3 \ldots \ldots \ldots \ldots \ldots$

E-3-SC-3. Steam Coal, Region $3 \ldots \ldots \ldots \ldots \ldots \ldots \ldots . \ldots \ldots$

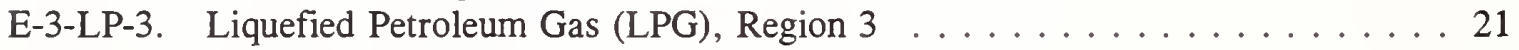

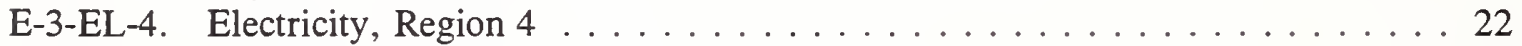

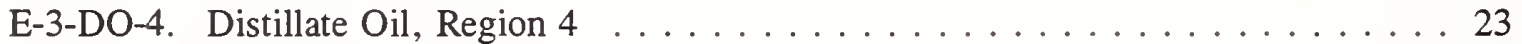

E-3-RO-4. Residual Oil, Region $4 \ldots \ldots \ldots \ldots \ldots \ldots$

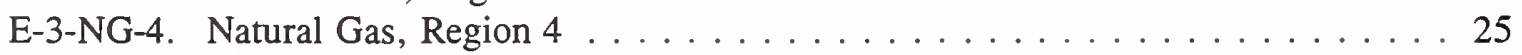

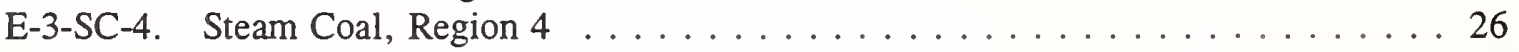

E-3-LP-4. Liquefied Petroleum Gas (LPG), Region $4 \ldots \ldots \ldots \ldots$. . . . . . . 27

E-3-EL-5. Electricity, U.S. Average . . . . . . . . . . . . . . . . . 28

E-3-DO-5. Distillate Oil, U.S. Average . . . . . . . . . . . . . . . . . . . 29

E-3-RO-5. Residual Oil, U.S. Average . . . . . . . . . . . . . . . . 30

E-3-NG-5. Natural Gas, U.S. Average . . . . . . . . . . . . . . . 31

E-3-SC-5. Steam Coal, U.S. Average ...................... 32

E-3-LP-5. Liquefied Petroleum Gas (LPG), U.S. Average . . . . . . . . . . . 33 
Part II. Tables of Present Worth Factors for Non-Energy Studies . . . . . . . . . . . . 34

Table Title

NE-1-1-ST. One-Time Costs, Zero Differential Escalation (Short-Term Studies) … . . 36

NE-1-1-LT. One-Time Costs, Zero Differential Escalation (Long-Term Studies) . . . . . . 37

NE-1-2-ST. One-Time Costs, Non-Zero Differential Escalation (Short-Term Studies) . . . . 38

NE-1-2-LT. One-Time Costs, Non-Zero Differential Escalation (Long-Term Studies) . . . . . 39

NE-2-1. Annually Recurring Costs, Zero Differential Escalation $(\mathrm{e}=0 \%) \ldots \ldots \ldots . .40$

NE-2-2. Annually Recurring Costs, Non-Zero Differential Escalation $(e=-5 \%) \ldots \ldots 41$

NE-2-3. Annually Recurring Costs, Non-Zero Differential Escalation $(e=-4 \%) \ldots . .42$

NE-2-4. Annually Recurring Costs, Non-Zero Differential Escalation $(e=-3 \%) \ldots . .43$

NE-2-5. Annually Recurring Costs, Non-Zero Differential Escalation $(e=-2 \%) \ldots . .44$

NE-2-6. Annually Recurring Costs, Non-Zero Differential Escalation $(\mathrm{e}=-1 \%) \ldots . .45$

NE-2-7. Annually Recurring Costs, Non-Zero Differential Escalation $(e=1 \%) \ldots . .46$

NE-2-8. Annually Recurring Costs, Non-Zero Differential Escalation $(e=2 \%) \ldots . .47$

NE-2-9. Annually Recurring Costs, Non-Zero Differential Escalation $(\mathrm{e}=3 \%) \ldots . .48$

NE-2-10. Annually Recurring Costs, Non-Zero Differential Escalation $(\mathrm{e}=4 \%) \quad \ldots . .49$

NE-2-11. Annually Recurring Costs, Non-Zero Differential Escalation $(e=5 \%) \ldots . .50$

Appendix A. Memorandum of Agreement on Criteria/Standards for

Economic Analysis/Life-Cycle Costing for MILCON Design . . . . . . . . 51 



\section{Part I. Tables of Present Worth Factors for Energy Studies}

Part I contains tables of present worth factors for use in computing the life-cycle costs of the competing alternatives in an energy-related study, in accordance with the provisions of governing DoD criteria (see Appendix A). These tables are all numbered in a sequence beginning with the letter " $E$ " for energy. The DOE discount rate used to compute these present worth factors is $4.1 \%$ (real, i.e., net of general inflation). This is the discount rate specified for use in energy-related projects by the Federal Energy Management Program for Fiscal Year (FY) 1996.

Table E-1, "Present Worth Factors--One-Time Costs, Zero Differential Escalation," provides present worth factors for costs which occur one time or at irregular intervals throughout the study period. These costs include construction/acquisition costs, non-annually recurring maintenance costs, major repair and replacement costs, and retention/salvage value or disposal cost. These factors are called "single present worth" (SPW) factors. The present worth of each cost

occurrence is found by multiplying that cost, in Date-of-Study (DOS) prices, by the SPW factor corresponding to the time of occurrence (years after DOS). Interpolation is encouraged for noninteger time periods.

Table E-2, "Present Worth Factors--Annually Recurring Non-Energy Costs, Zero Differential Escalation," provides present worth factors for all costs other than energy costs which are incurred annually throughout the study period in substantially the same amount each year (in constant dollar terms), such as routine maintenance and repair costs. These factors are called "uniform present worth" (UPW) factors. The factors in this table are based on the assumption that the DOS is in April 1996, the Beneficial Occupancy Date is in October of the same year or a future year, and that the annual cost occurs approximately at mid-year during each year of occupancy, or represents the sum of several costs distributed relatively uniformly throughout the year. The present worth of a cost recurring annually over the study period is found by multiplying the annual amount, in DOS prices, by the appropriate UPW factor. The number of payments generally corresponds to the number of years in the study period after the Beneficial Occupancy Date. Interpolation is encouraged for study periods and for beneficial occupancy dates other than those shown on the table.

Tables E-3-ET-R, where ET is the energy type code and $R$ is the region number $(R=5$ is for U.S. average), provide present worth factors for annually recurring energy costs. These factors are based on the assumption that annual energy usage/savings is constant from year to year, but that energy prices are changing over time, in accordance with the provisions of governing DoD criteria (see Appendix A). These factors are sometimes called "modified uniform present worth" (UPW*) factors. The UPW* factors in this table are based on the assumption that the DOS is in April 1996, the Beneficial Occupancy Date is in October of the same year or a future year, and that the annual energy cost occurs approximately at mid-year during each year of occupancy, or represents the sum of several costs distributed relatively uniformly throughout the year. The present worth of an annual energy cost over the study period is found by multiplying the annual amount, in DOS prices, by the appropriate UPW* factor. The number of payments generally corresponds to the number of years in the study period after the Beneficial Occupancy Date. Interpolation is encouraged for study periods and for Beneficial Occupancy Dates other than those shown on the tables. 


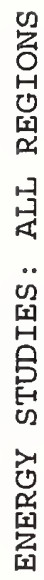

2
2
0
0
0
0
0

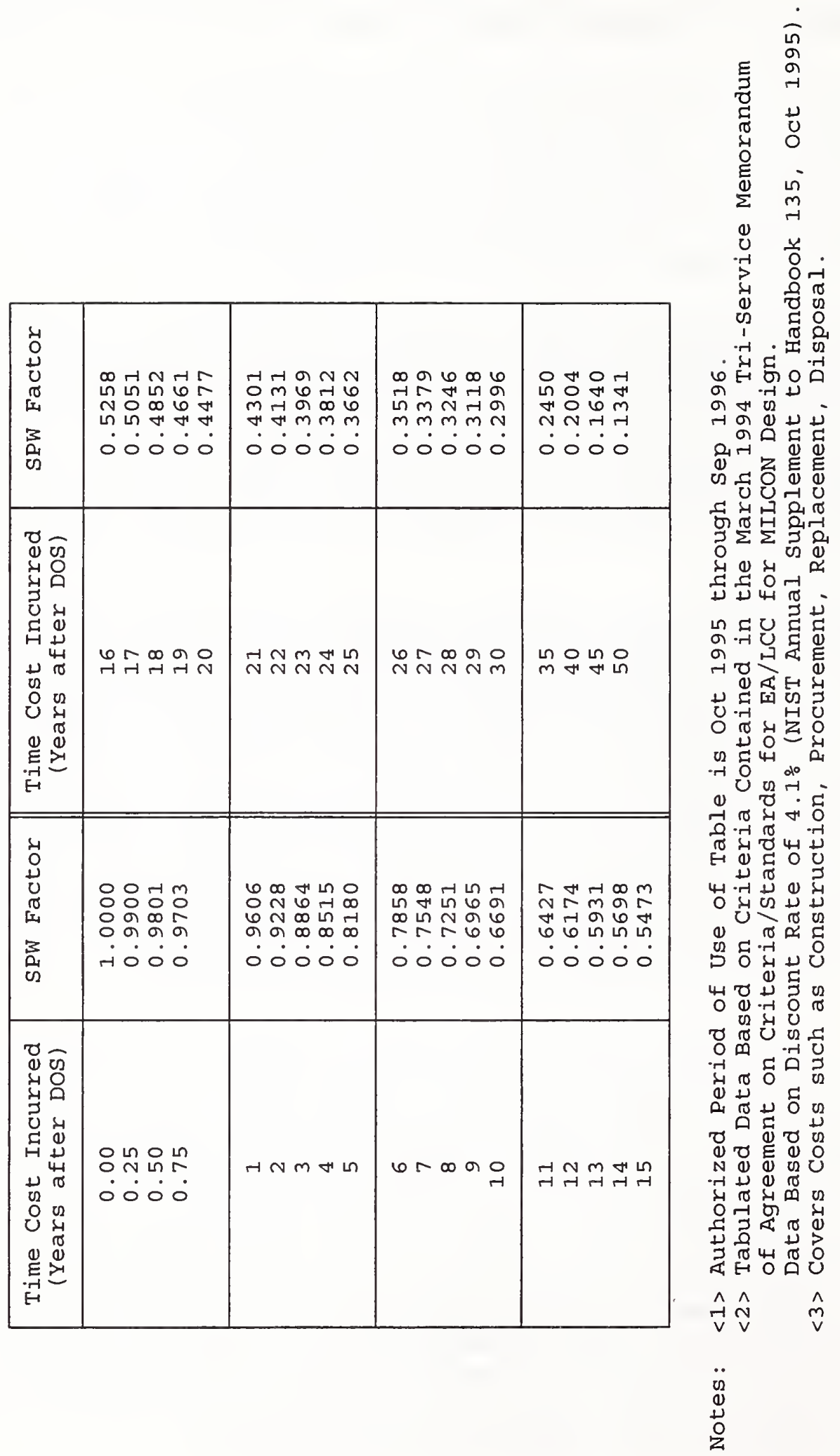

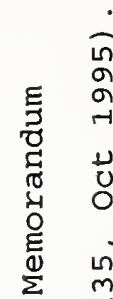

1

是

号

E

()

○烍

$\pi$

o 0

○ 回

U

$\pi$

ᄃ

प्र

34

崖

(1)

(1) 다

서 


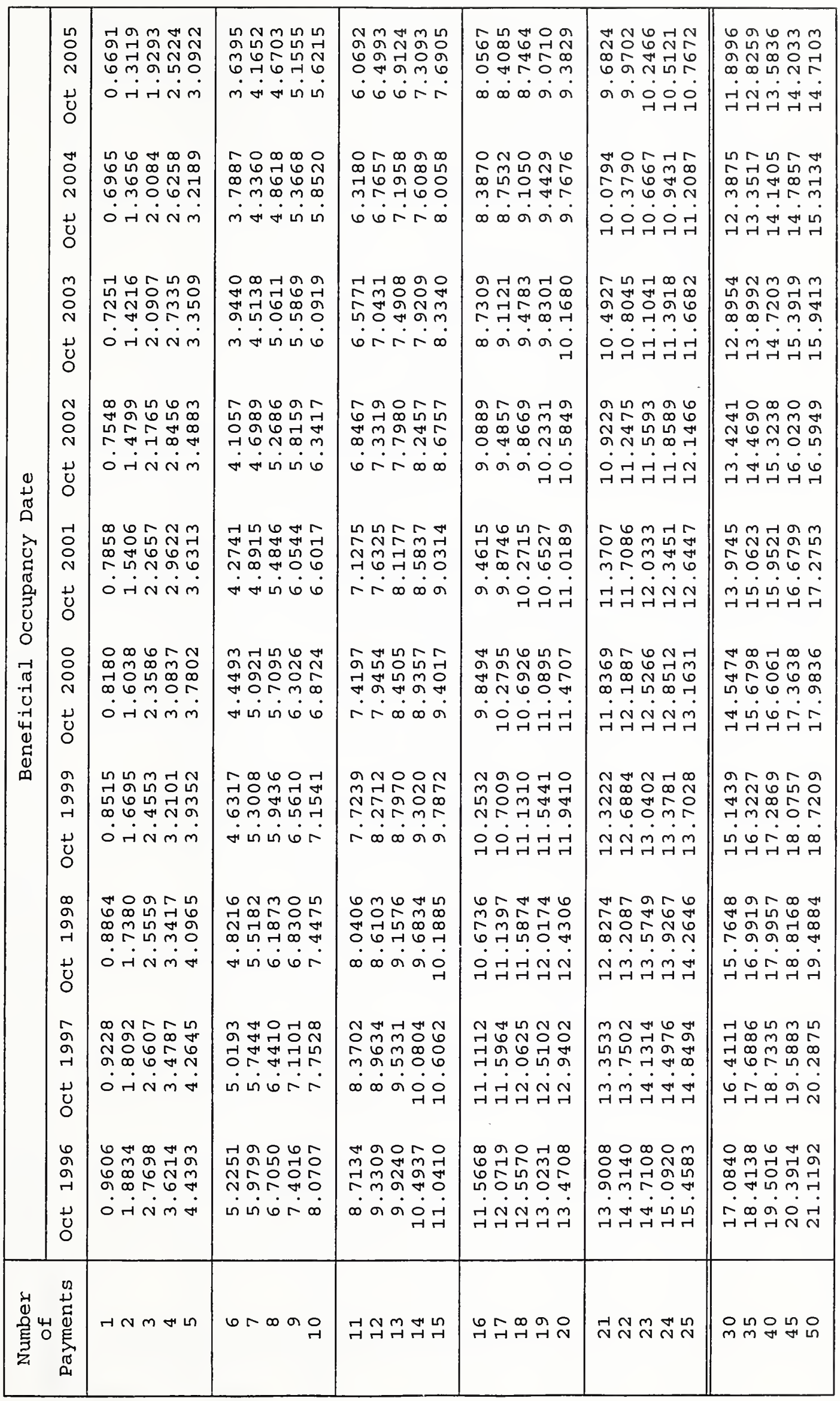

ब ने ङू ये 口马 ब들 ज本记 Uृ

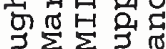
ठิ $\Sigma \sum$ 证 대 \& क क क म 14

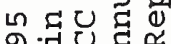
बे द तro 些昆。 U - 0 元 4 已

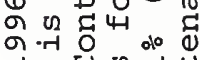

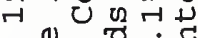
का 万人

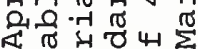

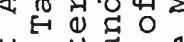

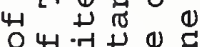
O क U 口舟

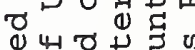
है이에 5 on 4

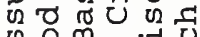
记 乐

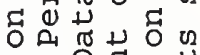
वृ वृ 0 व

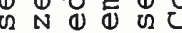

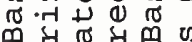
ต

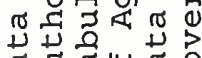

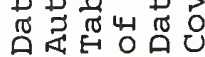
$\hat{\vec{v}} \hat{v} \quad \hat{m}$ 


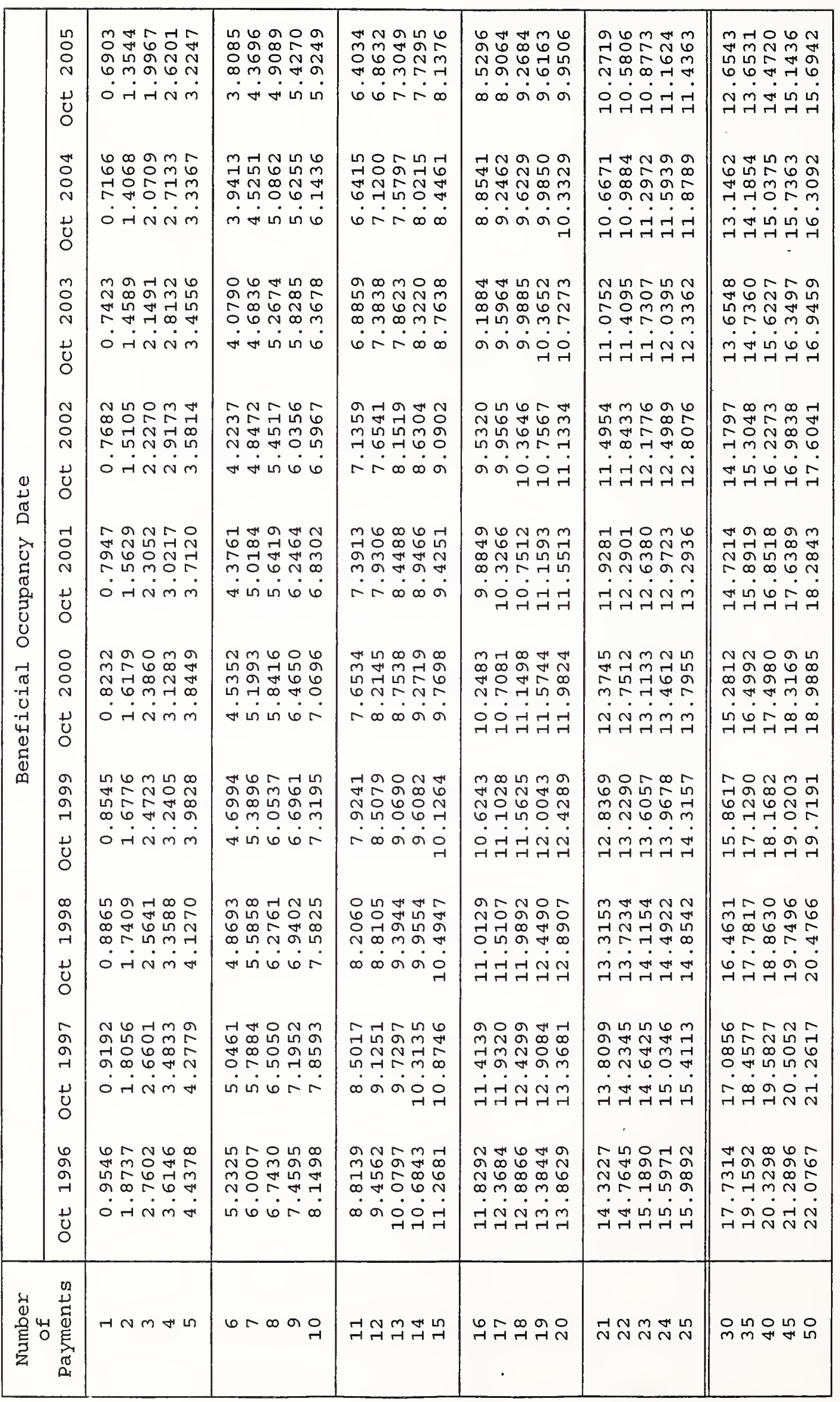




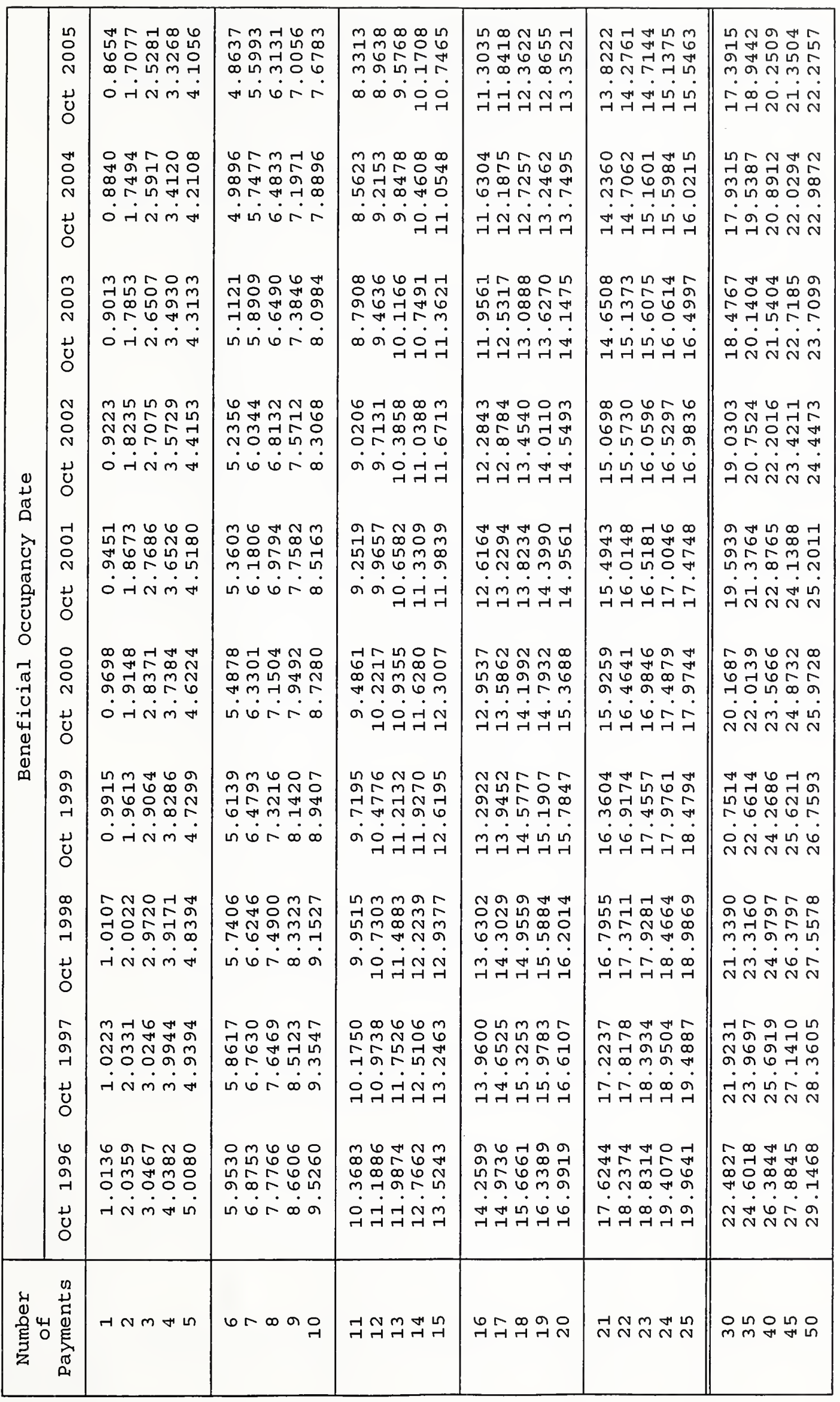

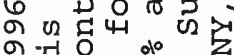
- 0 U 4

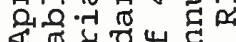
过

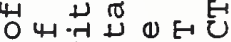
号的的 设

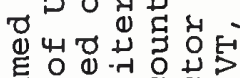

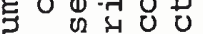

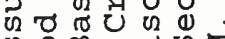

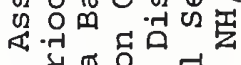
द岗 에 б б

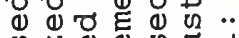
⿰ N 4 U

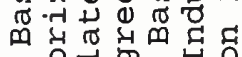
吕 ग

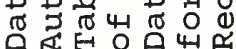
$\hat{\vec{v}} \hat{v} \quad \hat{v}$ 


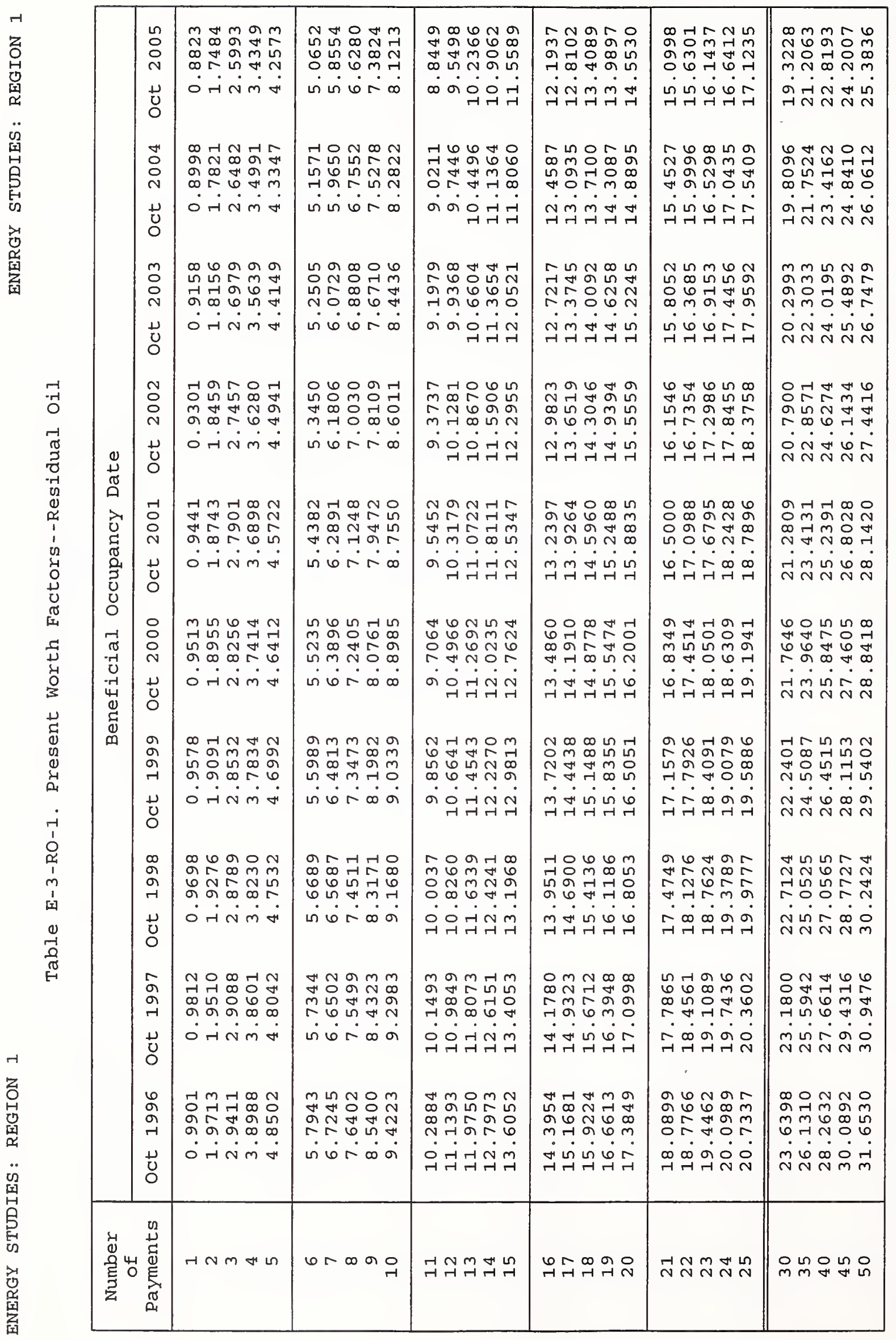

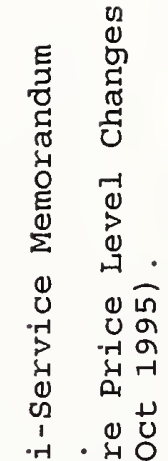

. С્ર ம बे तबू 담 0 낭

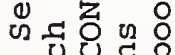
던ㄱㅁㅇㅁㅇ

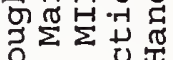
놈웜 ก द बन न्न ने

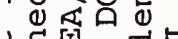

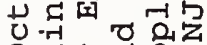
- 0 万ु

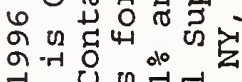
(1) च 듀

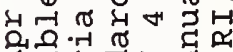
ब्र E O 至

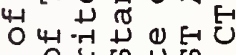

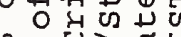
ภำ ص

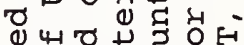

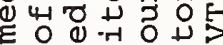

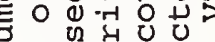

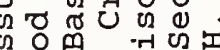

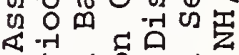
氙岀 ठ व Q 红 $N$ o थ

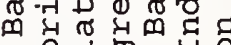
엄 워

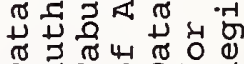
वर्ष $\hat{v} \hat{v} \quad \hat{v}$ $\ddot{0}$
0
$u$
0
$z$ 


\begin{tabular}{|c|c|c|c|c|c|c|c|}
\hline \multirow{10}{*}{ 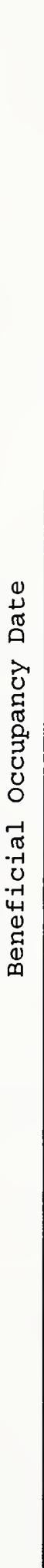 } & $\begin{array}{l}n \\
0 \\
0 \\
\text { N } \\
y \\
U \\
0\end{array}$ & 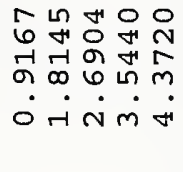 & 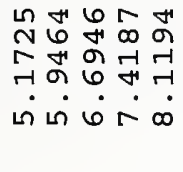 & 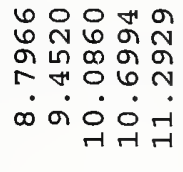 & 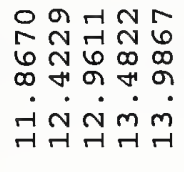 & 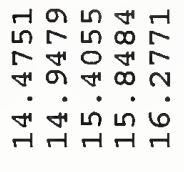 & 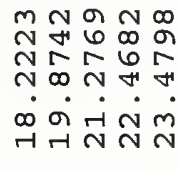 \\
\hline & 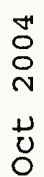 & 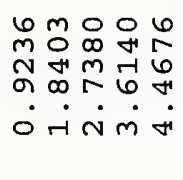 & 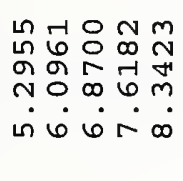 & 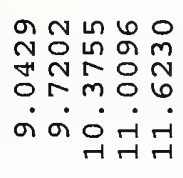 & 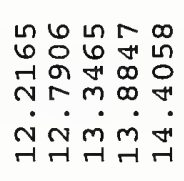 & 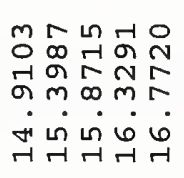 & 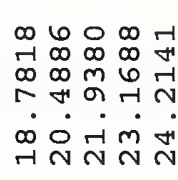 \\
\hline & $\begin{array}{l}m \\
0 \\
0 \\
\sim \\
+ \\
u \\
0\end{array}$ & 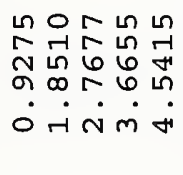 & 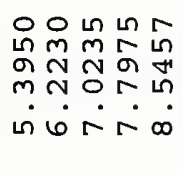 & 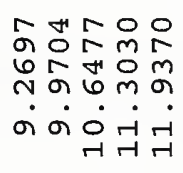 & 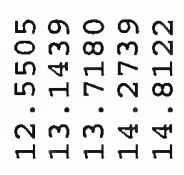 & 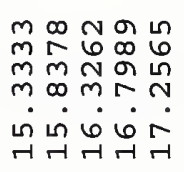 & 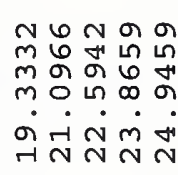 \\
\hline & $\begin{array}{l}\text { N } \\
0 \\
0 \\
N \\
+U\end{array}$ & 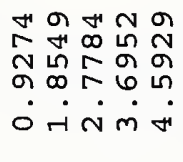 & 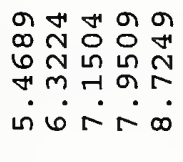 & 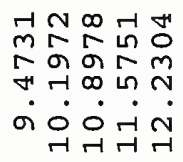 & 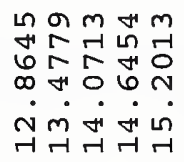 & 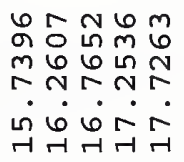 & 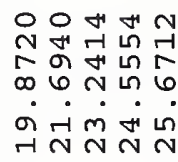 \\
\hline & 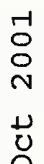 & 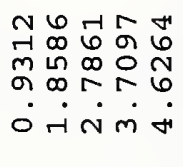 & 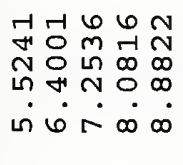 & 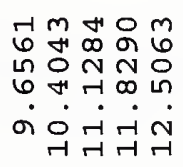 & 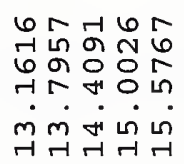 & 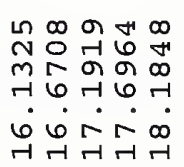 & 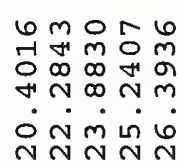 \\
\hline & $\begin{array}{l}0 \\
0 \\
0 \\
\text { N } \\
+U \\
0\end{array}$ & 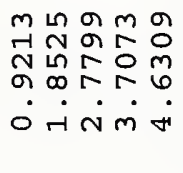 & 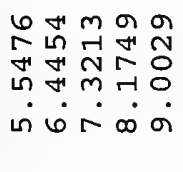 & 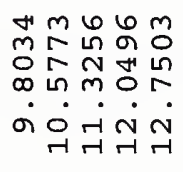 & 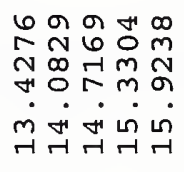 & 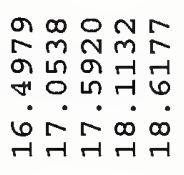 & 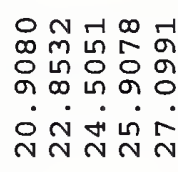 \\
\hline & $\begin{array}{l}\text { o } \\
\sigma \\
\sigma \\
r \\
+ \\
u \\
0\end{array}$ & 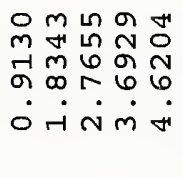 & 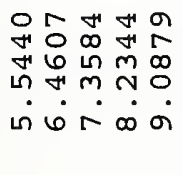 & 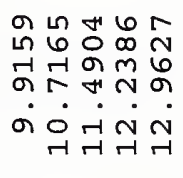 & 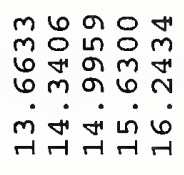 & 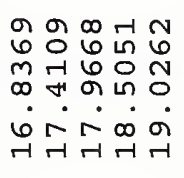 & 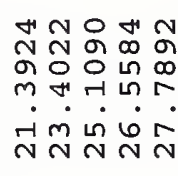 \\
\hline & 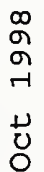 & 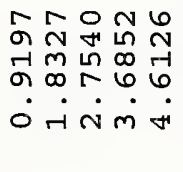 & 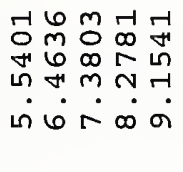 & 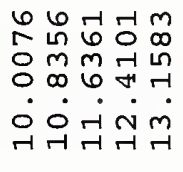 & 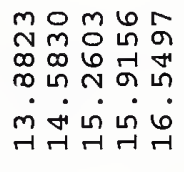 & 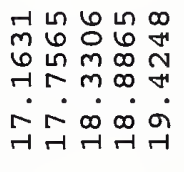 & 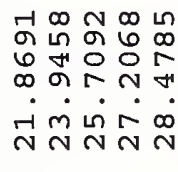 \\
\hline & 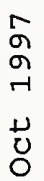 & 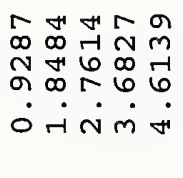 & 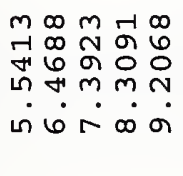 & 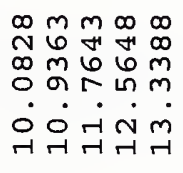 & 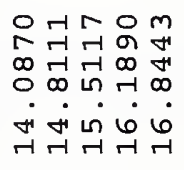 & 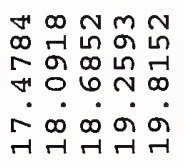 & 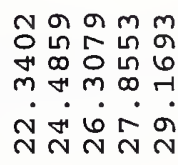 \\
\hline & 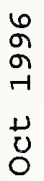 & 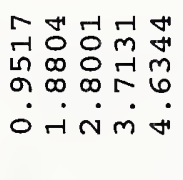 & 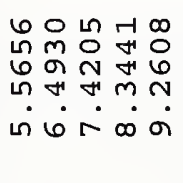 & 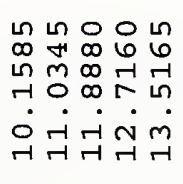 & 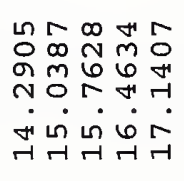 & 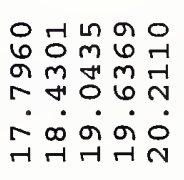 & 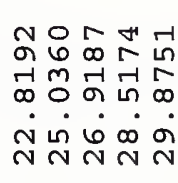 \\
\hline 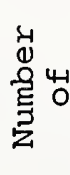 & 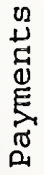 & $H N M$ & 6N $\infty 00$ & 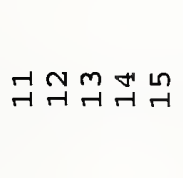 & $\begin{array}{l}6 \sim \infty \\
\text { 듀 }\end{array}$ & $\stackrel{-}{\sim} \stackrel{\sim}{N} \stackrel{\sim}{\sim} \stackrel{\sim}{\sim}$ & 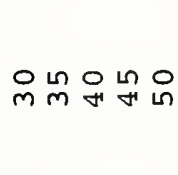 \\
\hline
\end{tabular}

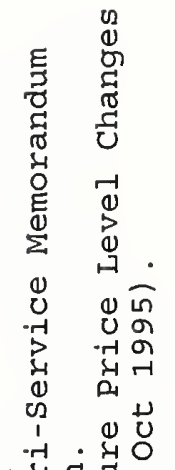
-

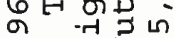
बे भूय न ब व ब릉 的 थ

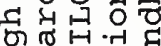

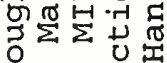
엉 40

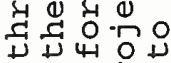
เी द्रिम बैन्तల म の 됭

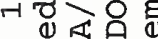

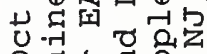
- О त म

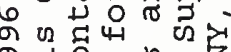

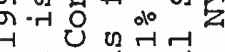
乔 $\sigma$ 它

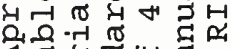

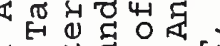

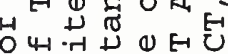

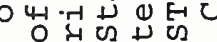

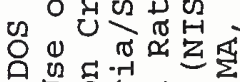
๑

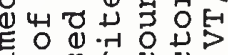

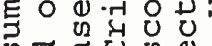

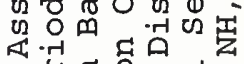
द ปี 口告出 (1) प्ष U $\mathbb{N}$ Q ๙

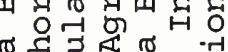

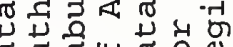

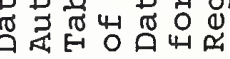
$\hat{i} \hat{v} \quad \hat{v}$ in 


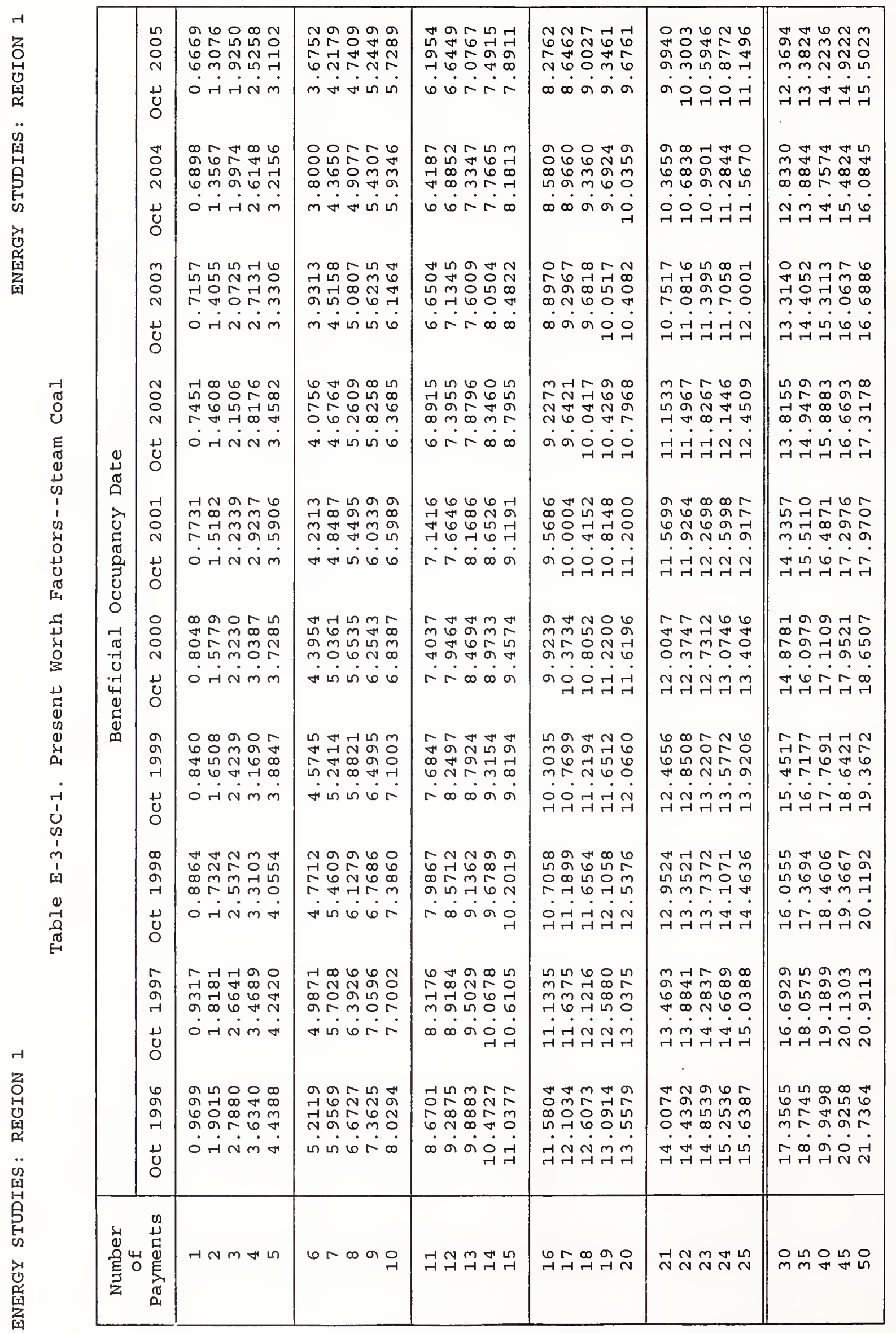

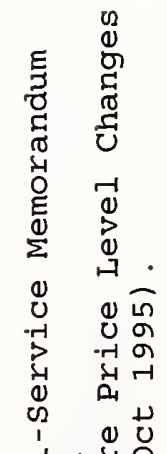

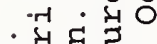

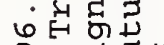

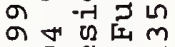

तने बे त

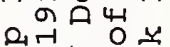

ब도응

บิ

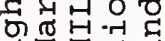

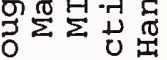

过先告。

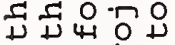

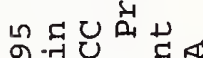

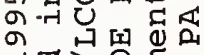
न \&

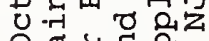
- 0 म

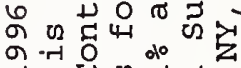
ने 0 थ

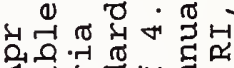

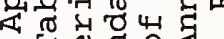
山出䒕正 ○出苟嵌界 ข

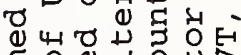
ह कृ

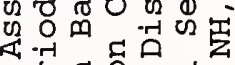
㝳 ᄃ ㅆ ช Q \& थ $N$ \& 02 药 ०ू

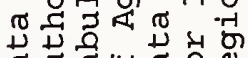
ช $\hat{i} \hat{v} \quad \hat{m}$ نू 
ลे ํํํํํ걷 a 근 7 这.

ㄴํำ ०नना

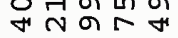

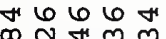

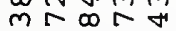
a 6 mor angir

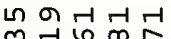

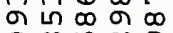
○ा เท เก

마저 ถัตूत กับ ᄂก जं

(1)

orma Non 0 เก

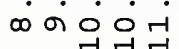
กं

$m m-1$ 눙요 ดूิ ชิ N $\infty$ L बंवंनं

an $\cos$

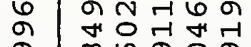
ने क्ष $\rightarrow$ बून

06 ㄴ 60 ᄂึ

N $N$ N

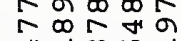

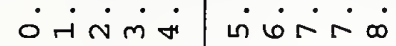
सम ब우궁

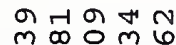
Nivi

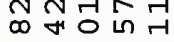

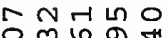
ठलख요 0
0
0 तेंm

N은 $m \infty$ अ N अ तुलिभुन्त

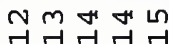
ปै 崩出 नतन त4न4त

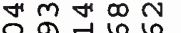

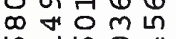

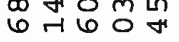
$\forall$ iो का 6

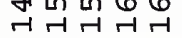

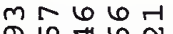
约 - 6 นก เก 6 or तन

N ले अने 6 न 600 نं तन
는 느요

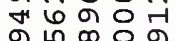
कं $\rightarrow$ N $N \mathrm{~N}$

은 느 6 ฟ in $\mathrm{N}$ C⿺辶 कं $\operatorname{tnnN}$

\section{$n$}

in
a

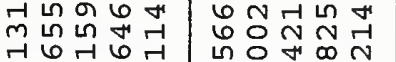




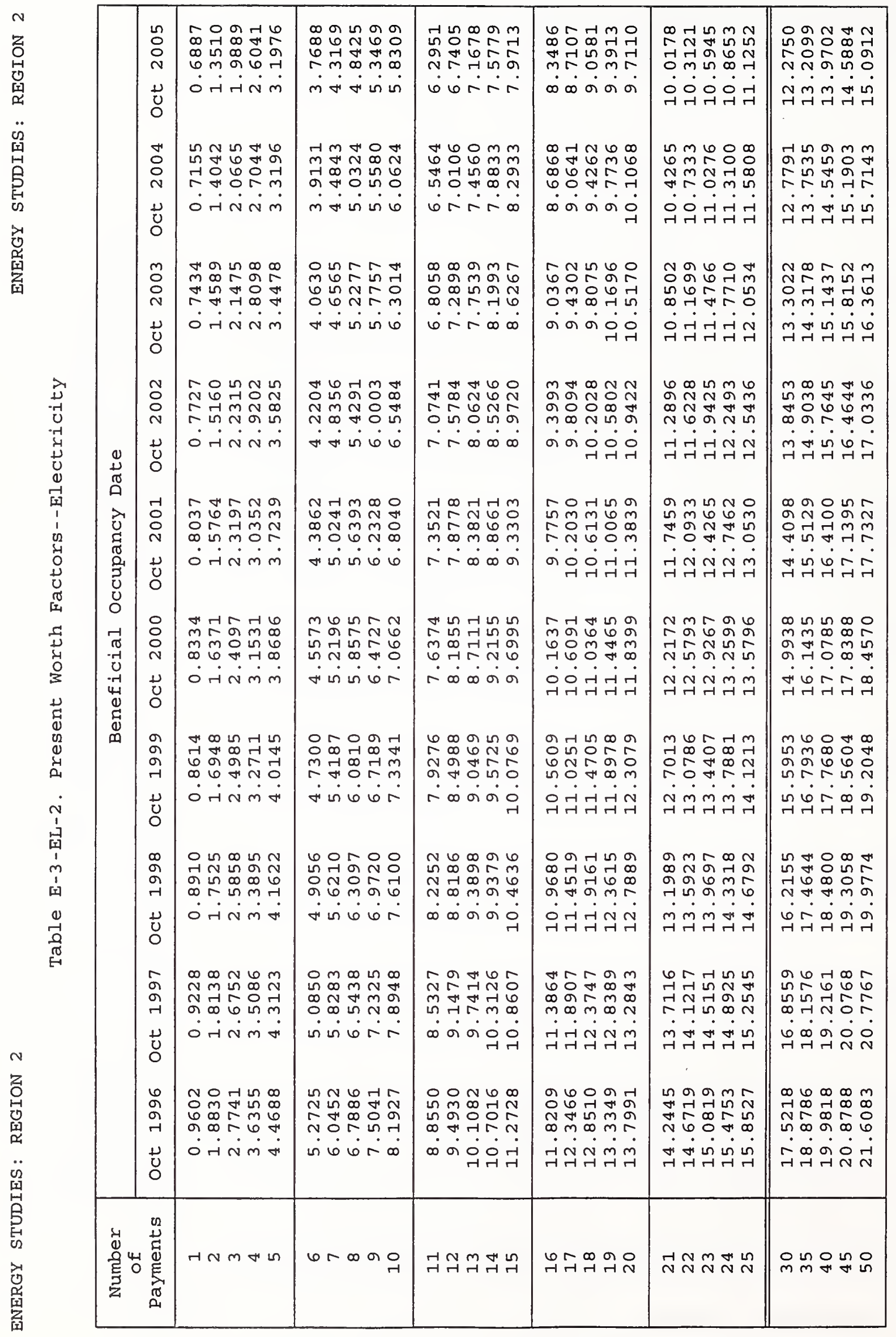

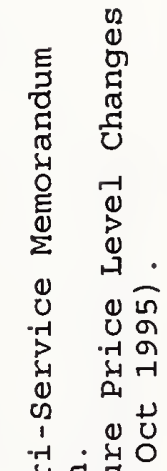

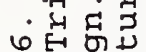

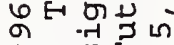
नु बूर न के बैँ

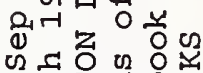
थ 덩웡

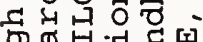

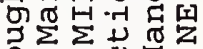
O

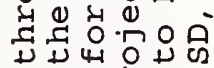
ก $匚$ 品

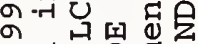
당ํㅇ 近需。

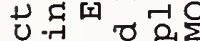
. О

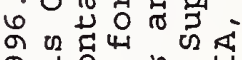
का न्न 0 d० तथ 0 थ

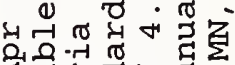

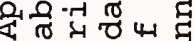
ED更O

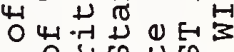
○ण कण का \% ص

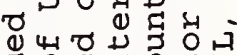

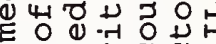
उ ए क थ2 ठठ

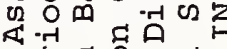
द ○元岂 ठ U ๙

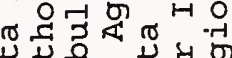
से

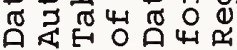
$\stackrel{\hat{v}}{\hat{v}} \quad \hat{m}$ 


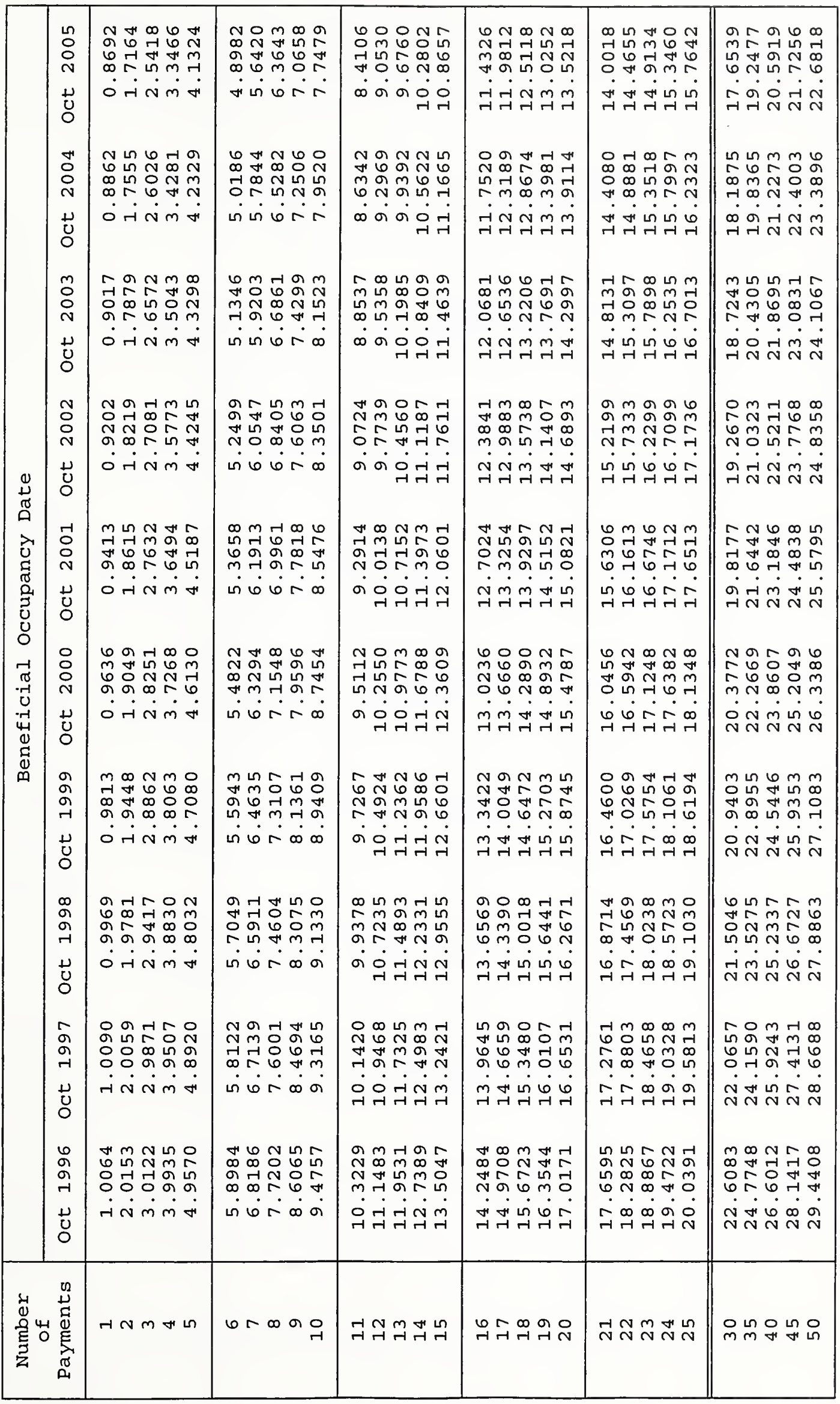

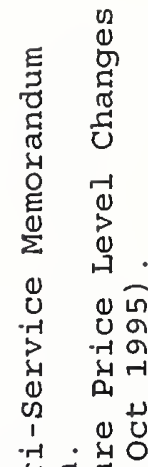
近是

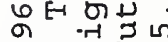
बे भुज तबष बत्ति 过

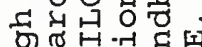

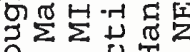
प्र

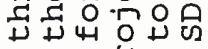
约的品 बून्न 山 舀

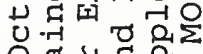
- 0 त्र 4 है 次若出 ने ${ }^{-1}$ ठ तथ वृत

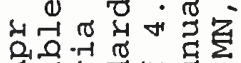

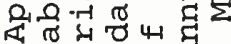

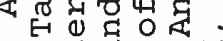

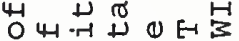
岁出岁号兒

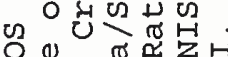

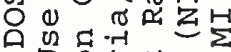
口可牙山

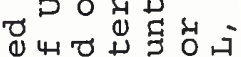

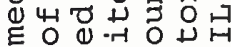

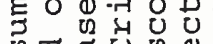
थ

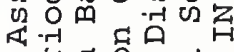
द प्山

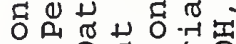
ه ه ब U $N$ व

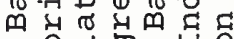
ต मू శ్

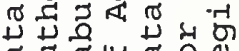

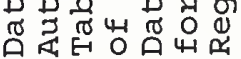
$\hat{\vec{v}} \hat{v} \quad \hat{m}$ 00
0
0
0 


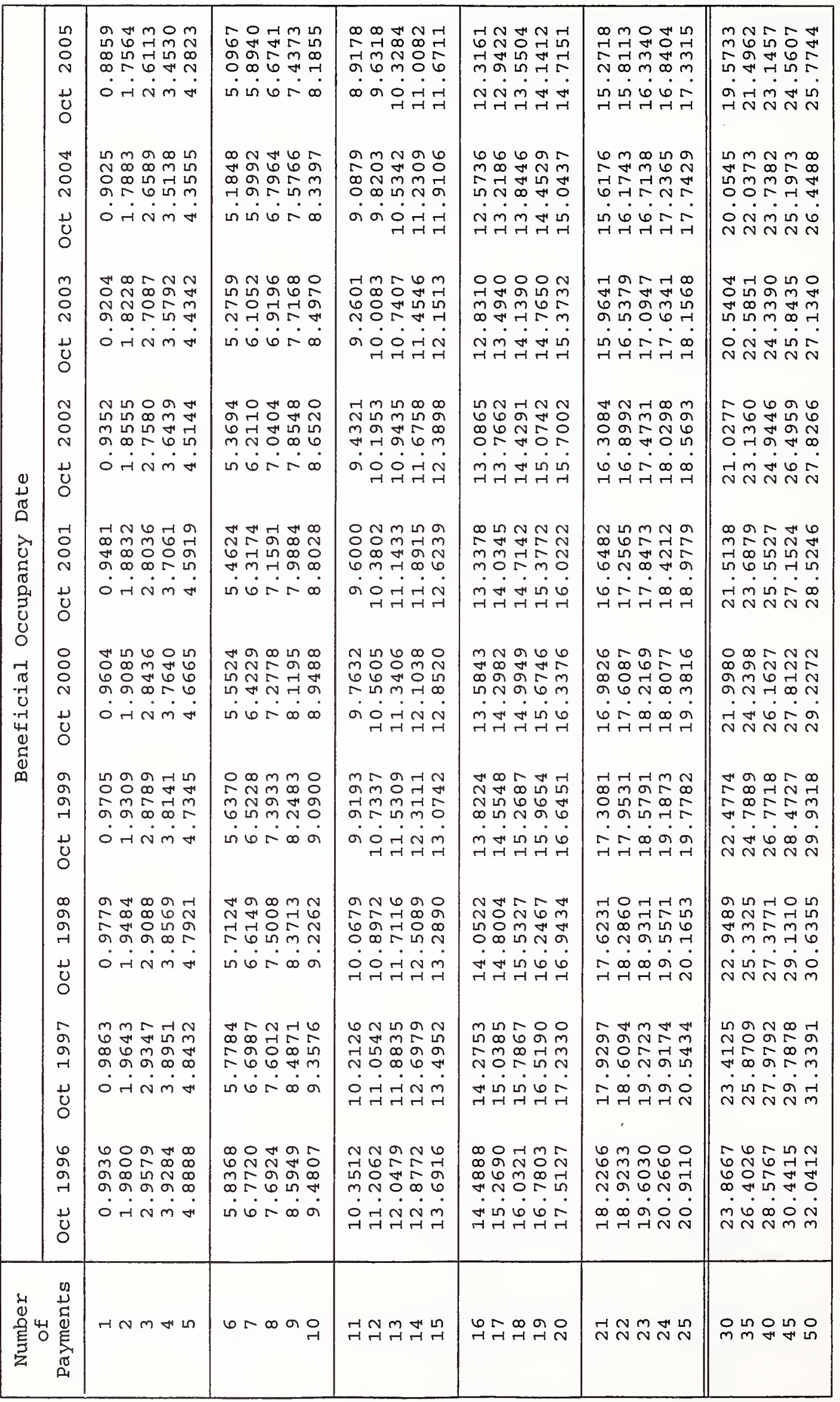

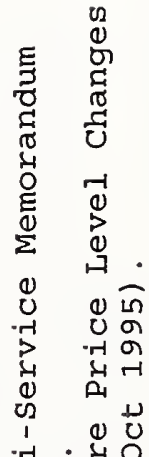

न

6ै हु

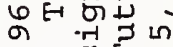

बे णू 记

न बे एँ

कृ

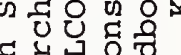

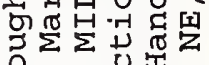

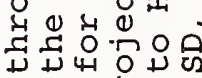

几 दU म

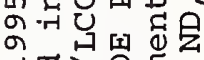

न

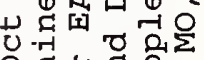

० 0 \%

ดั य

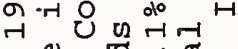

4

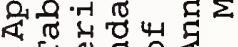

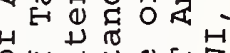
出出吕O

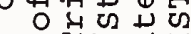

ण 0 ठ

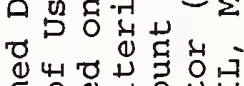
E 0 \& 今

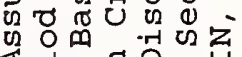

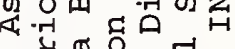

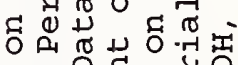
只运 वण 0 \& थ $N$ \& 02 ๗ొ 口엄

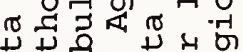

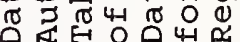

$\hat{v} \hat{v} \quad \hat{v}$

$\ddot{n}$
0
0
0
$z$ 


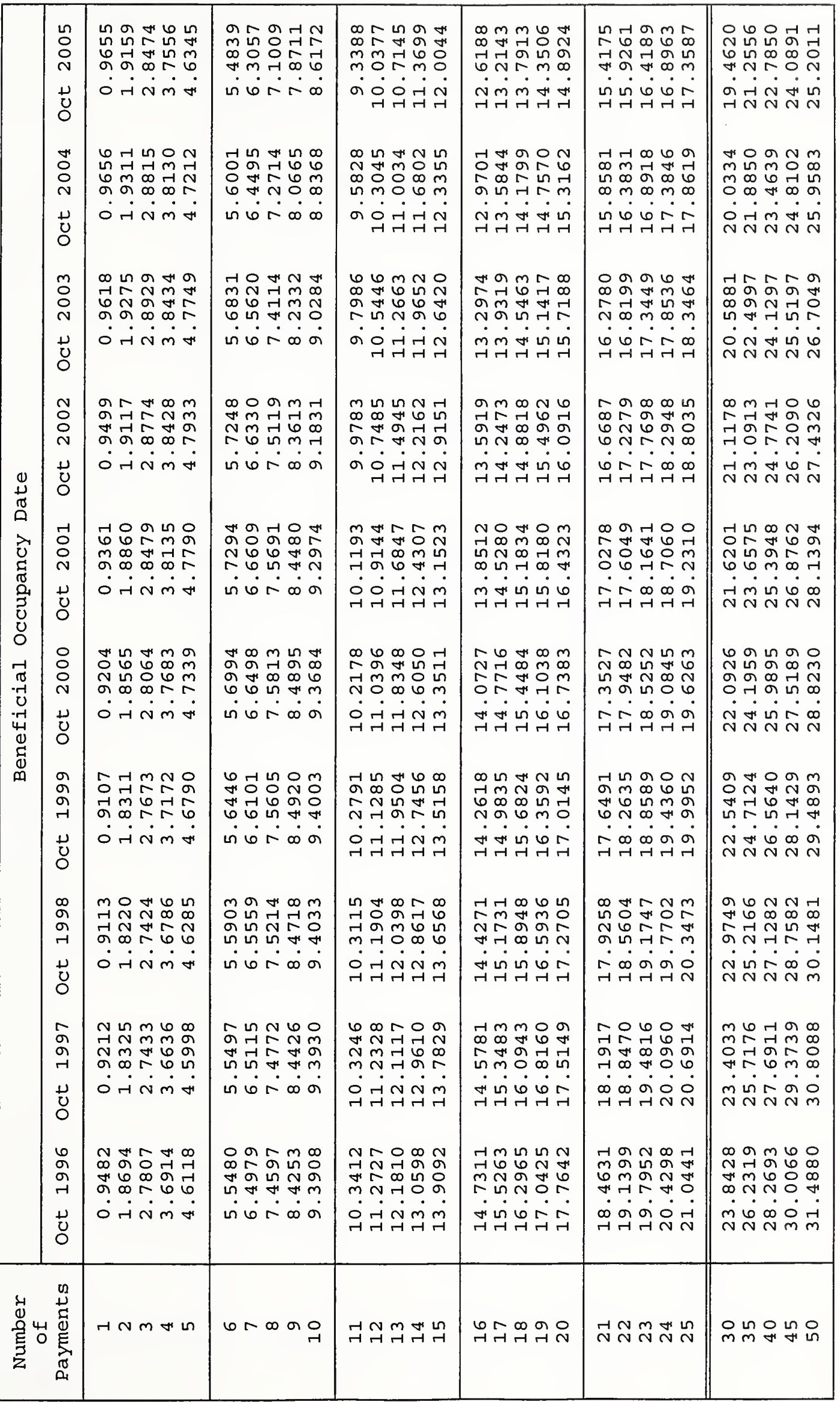

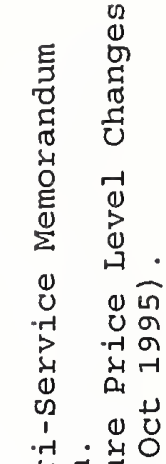

ic 4

6 स बे णु तब

बनि फै ๗ ठ บ द 息幽易

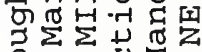
O

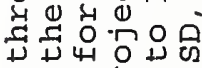
ก

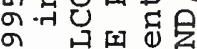
न

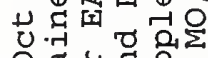

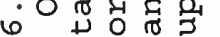

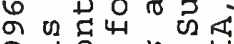
ब.

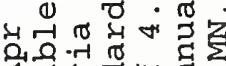

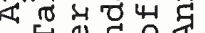
世界贾 0 出

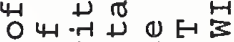

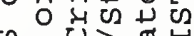

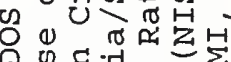

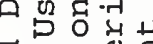
ס

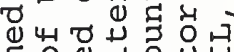
ह 0 品 0 ज्ञ

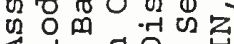
यन्न घ口

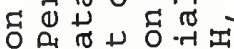
מ व

(1) ठ ह \& 0

⿰纟 $N$ \& 02

๓

以 म्न

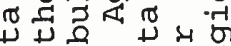

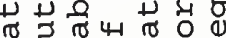

㲎告出告出

$\hat{v} \hat{v} \quad \hat{m}$

0
0
1
0
$Z$ 


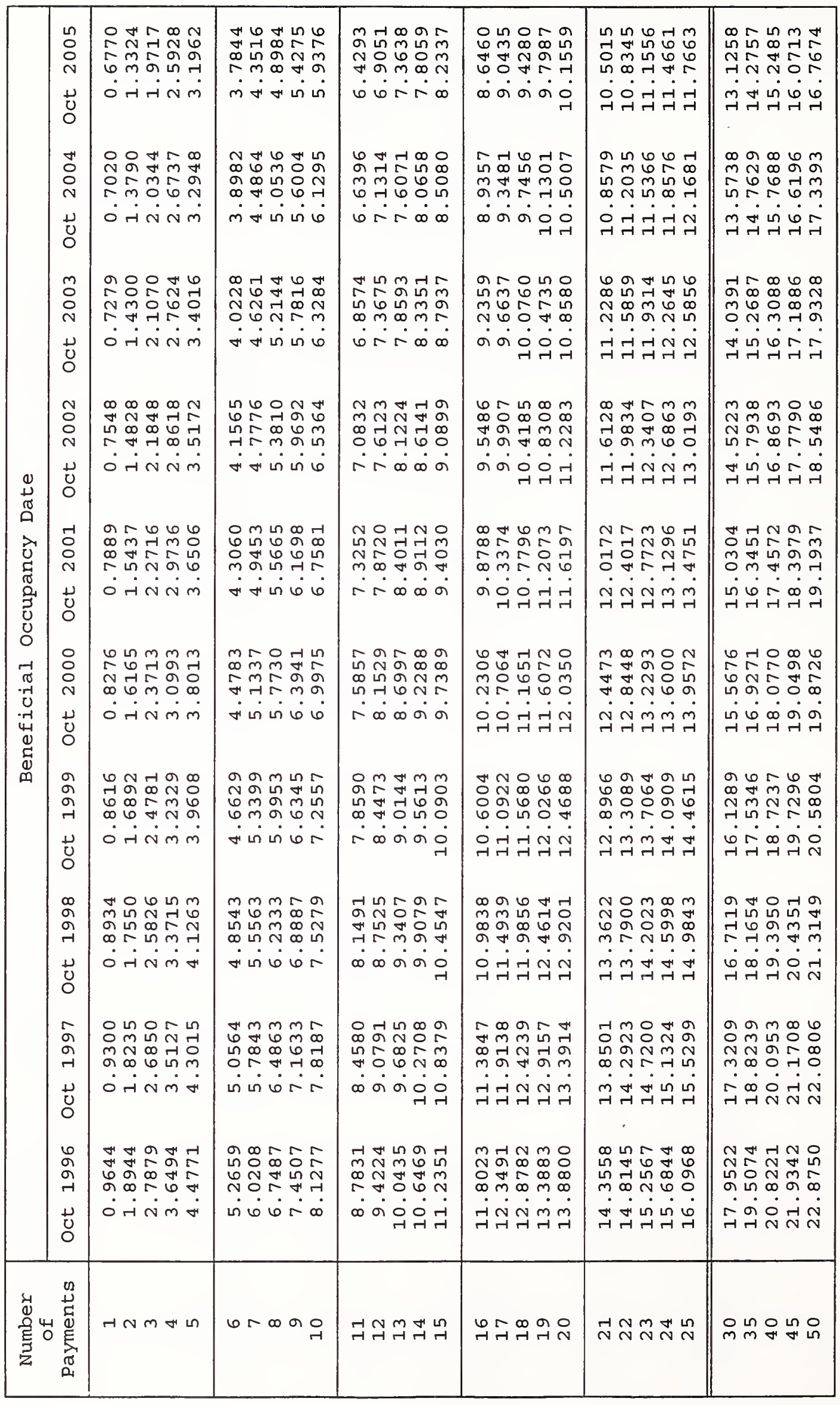

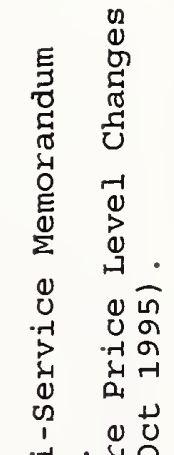

.

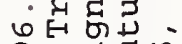

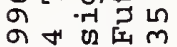

ने है क्य

م,

बैद

थ

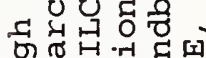

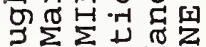

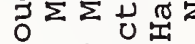

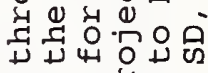

纪台

बून्म 됭

ने

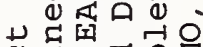

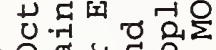
- 0 尔

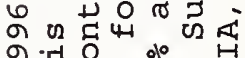

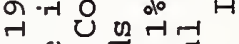

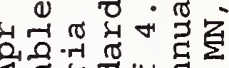
बत्रण प्र H O世 毕的昆

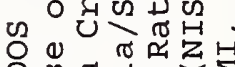

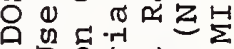
四

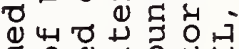

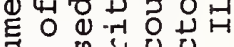
蛇 近.

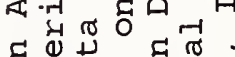
ธี ช ه

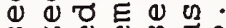
V2 N \& 0 ⿰讠

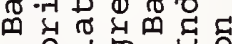
ช

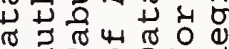

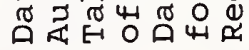
$\hat{\imath} \stackrel{\hat{v}}{\hat{v}} \hat{v}$ 雚 


\begin{tabular}{|c|c|c|c|c|c|c|c|}
\hline \multirow{11}{*}{ 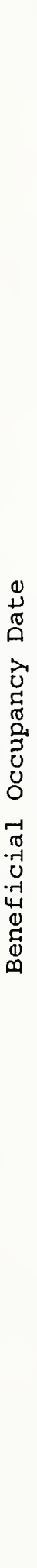 } & $\begin{array}{l}\text { L } \\
0 \\
0 \\
\text { v } \\
\nu \\
0 \\
0\end{array}$ & 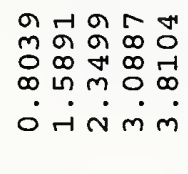 & 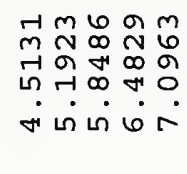 & 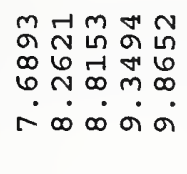 & 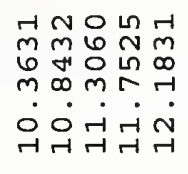 & 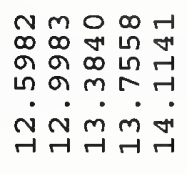 & 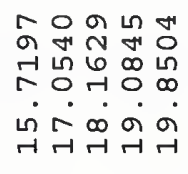 \\
\hline & $\begin{array}{l}\stackrel{+}{0} \\
0 \\
\text { N } \\
+ \\
0\end{array}$ & 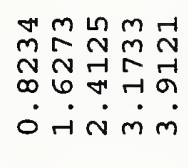 & 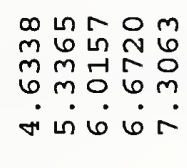 & 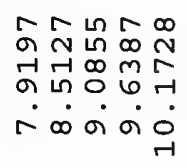 & 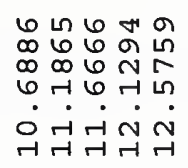 & 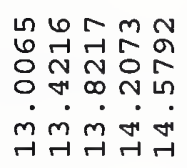 & 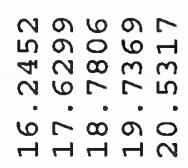 \\
\hline & 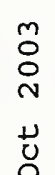 & 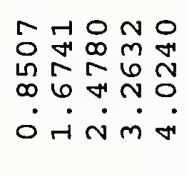 & 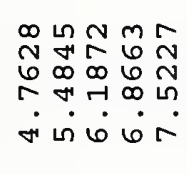 & 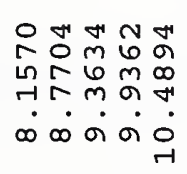 & 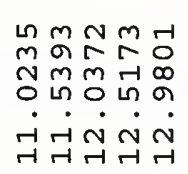 & 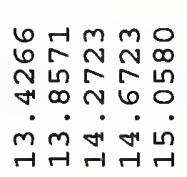 & 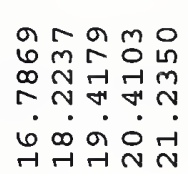 \\
\hline & $\begin{array}{l}\text { O } \\
\text { O } \\
\text { N } \\
\stackrel{\nu}{U} \\
0\end{array}$ & 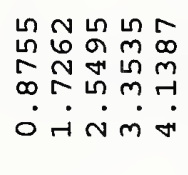 & 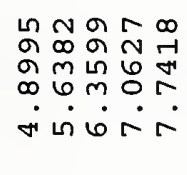 & 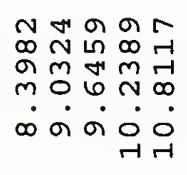 & 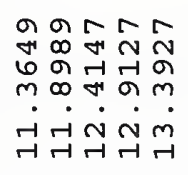 & 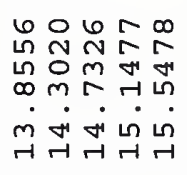 & 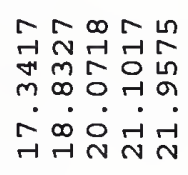 \\
\hline & $\begin{array}{l}-1 \\
\circ \\
0 \\
N \\
\nu \\
0 \\
0\end{array}$ & 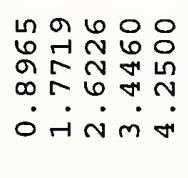 & 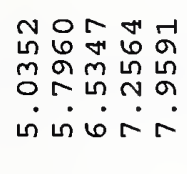 & 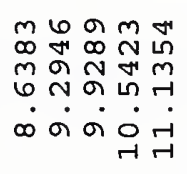 & 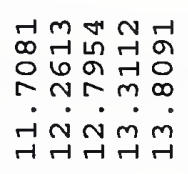 & 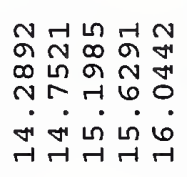 & 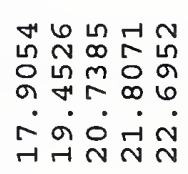 \\
\hline & 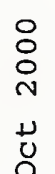 & 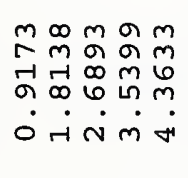 & 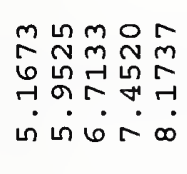 & 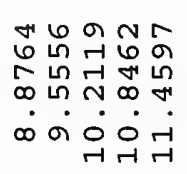 & 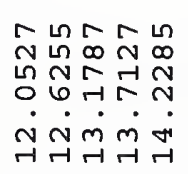 & 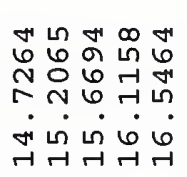 & 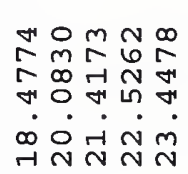 \\
\hline & $\begin{array}{l}\text { ने } \\
\sigma \\
\text { r } \\
\dot{y} \\
0 \\
0\end{array}$ & 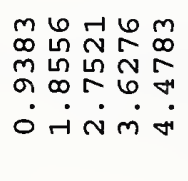 & 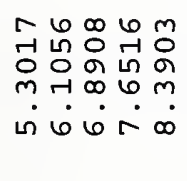 & 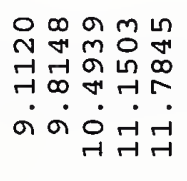 & 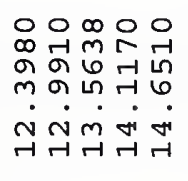 & 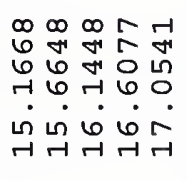 & 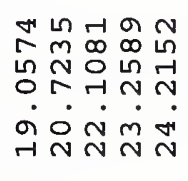 \\
\hline & $\begin{array}{l}\infty \\
\sigma \\
\sigma \\
r \\
1 \\
0\end{array}$ & 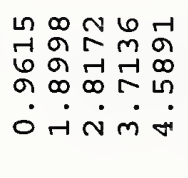 & 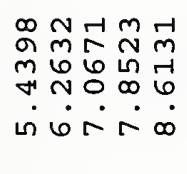 & 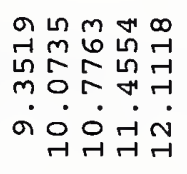 & 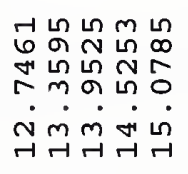 & 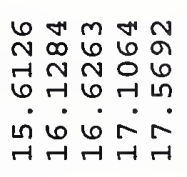 & 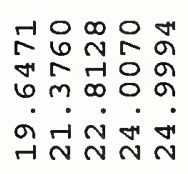 \\
\hline & $\begin{array}{l}\hat{\sigma} \\
\text { aे } \\
r \\
1\end{array}$ & 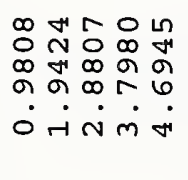 & 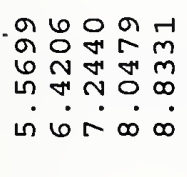 & 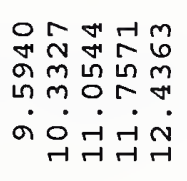 & 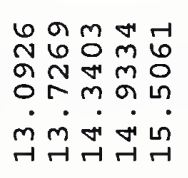 & 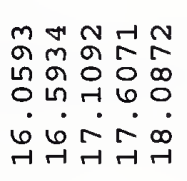 & 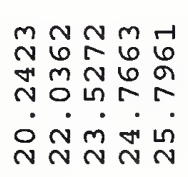 \\
\hline & $\begin{array}{l}6 \\
2 \\
\stackrel{-}{r} \\
-1\end{array}$ & 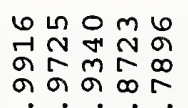 & 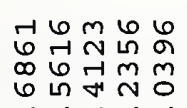 & 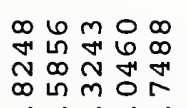 & 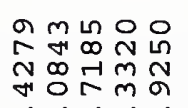 & $\begin{array}{l}\infty \\
\infty\end{array}$ & 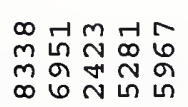 \\
\hline & $\stackrel{\bigsqcup}{0}$ & $0-N m \pi$ & เ $6 \pi \infty$ & 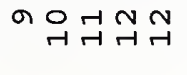 & ભિ & 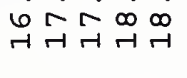 & $\stackrel{N}{N} \stackrel{\sim}{N} \stackrel{6}{N}$ \\
\hline 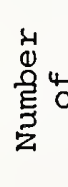 & $\vec{c}$ & HNM & 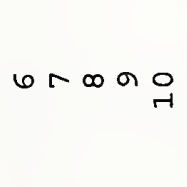 & 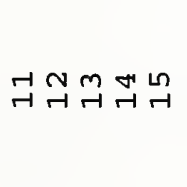 & 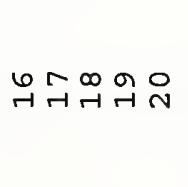 & $\stackrel{\sim}{\sim} \underset{N}{\sim} \stackrel{M}{\sim} \stackrel{\sim}{\sim}$ & 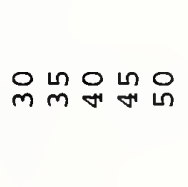 \\
\hline
\end{tabular}

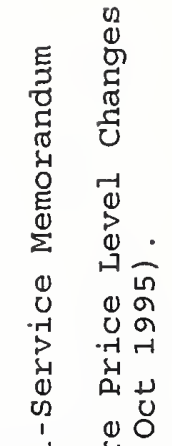

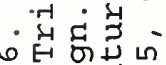

ดั

ने ण任

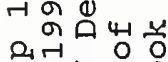

ब्न

थ

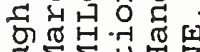

วิ沙要至

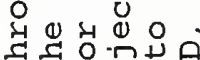

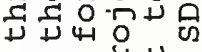
纪台岩 م.न

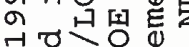

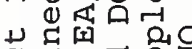

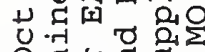

- $\bigcirc$ \% म

ด้ 的范柴 नेन्न है न 4

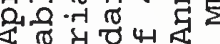
$\rightarrow 00$

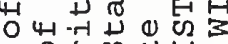
Л 政 ช0

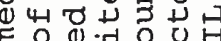

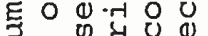
काष 0. 近每 ธ์ क म ठ ठ

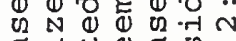

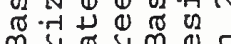

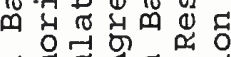
ธ

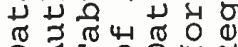

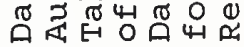
$\hat{v} \stackrel{\hat{v}}{\hat{v}} \quad \hat{v}$ in 


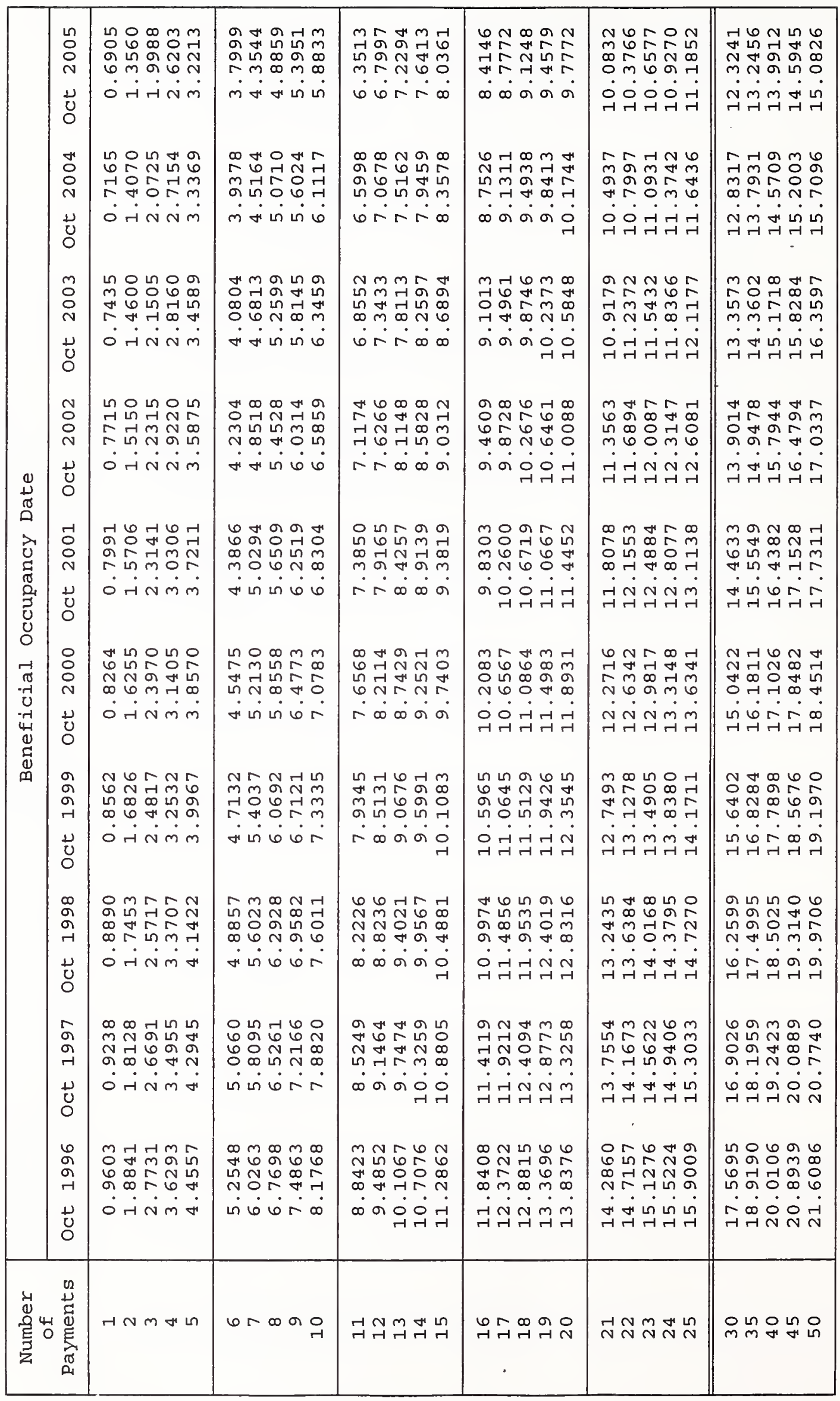

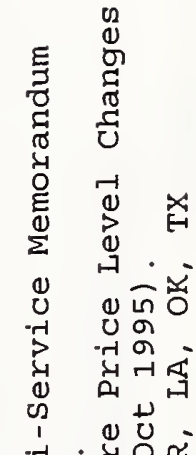

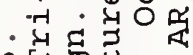

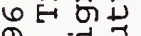

बान्त 510 तु बूल तथ ने

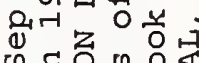
凹 의

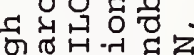

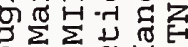
วิ $\Sigma \Sigma \nu_{0}^{\pi}$

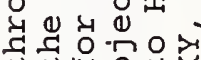

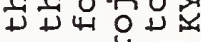
ต बैन्नट म त

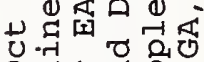
प न ดे यूट

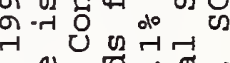

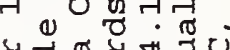
भ다유

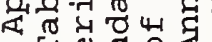

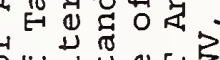
कफ ○प्य

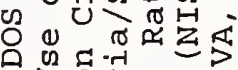

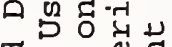
ช

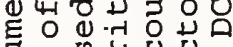

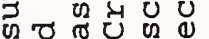

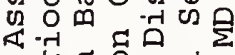
ब. त्र โ ช Q 0 过

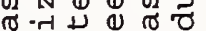
ต ๘

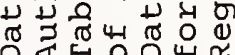
$\hat{v} \stackrel{\hat{v}}{\hat{v}} \quad \hat{v}$ $\ddot{0}$ 


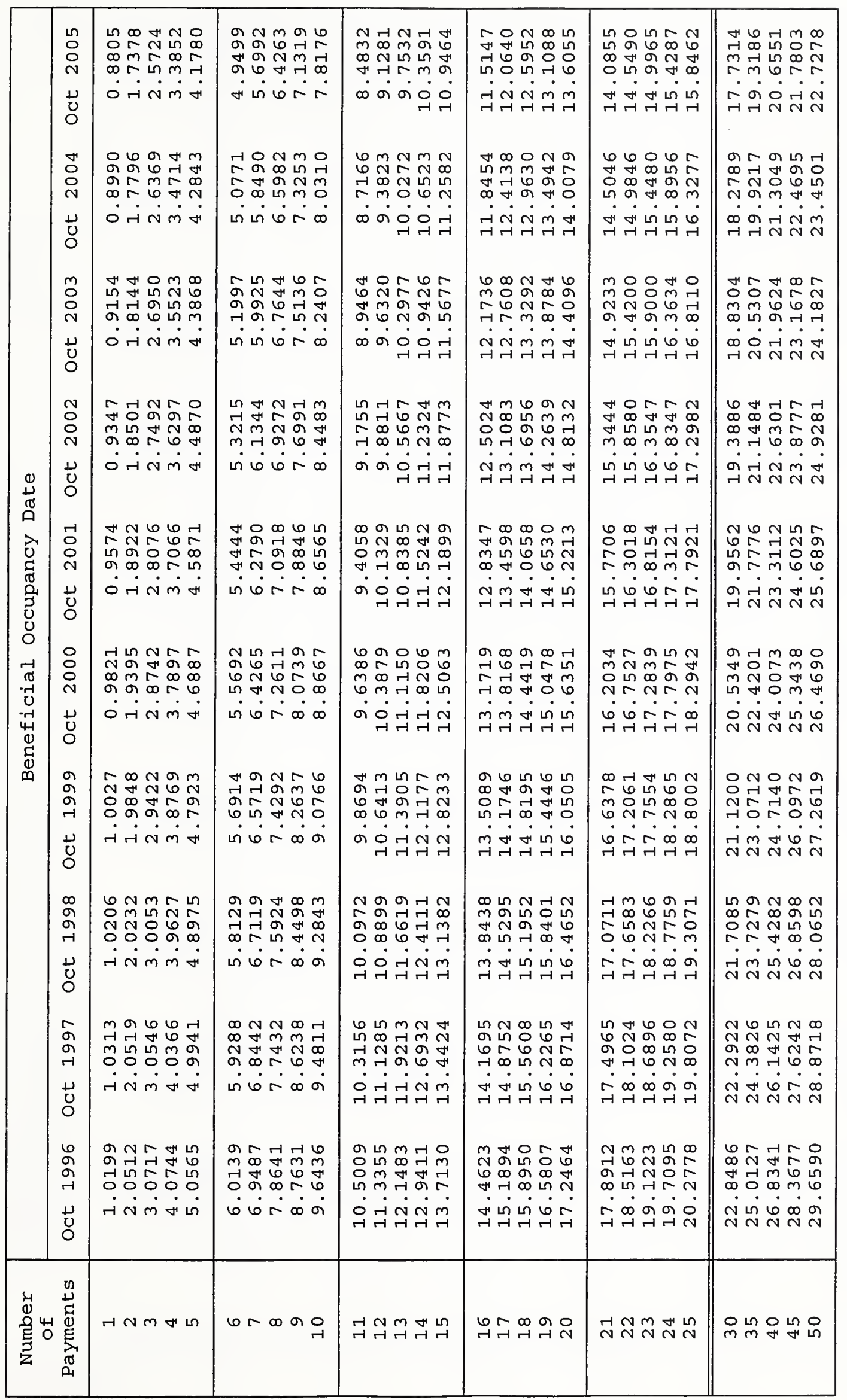

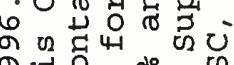
नेन ठ ठ ग (1)

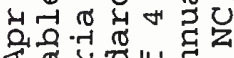
过望出

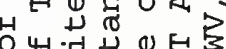
岑近崩㞻昆

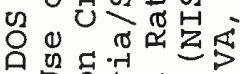
ช 0 岁岂

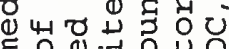
ह 0 ब 疑 0

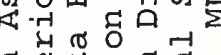
ปี व

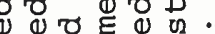
U $N$ \& ब U

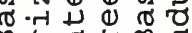
๓ ๙

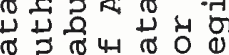

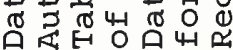
$\hat{v} \hat{v} \quad \hat{v}$ 


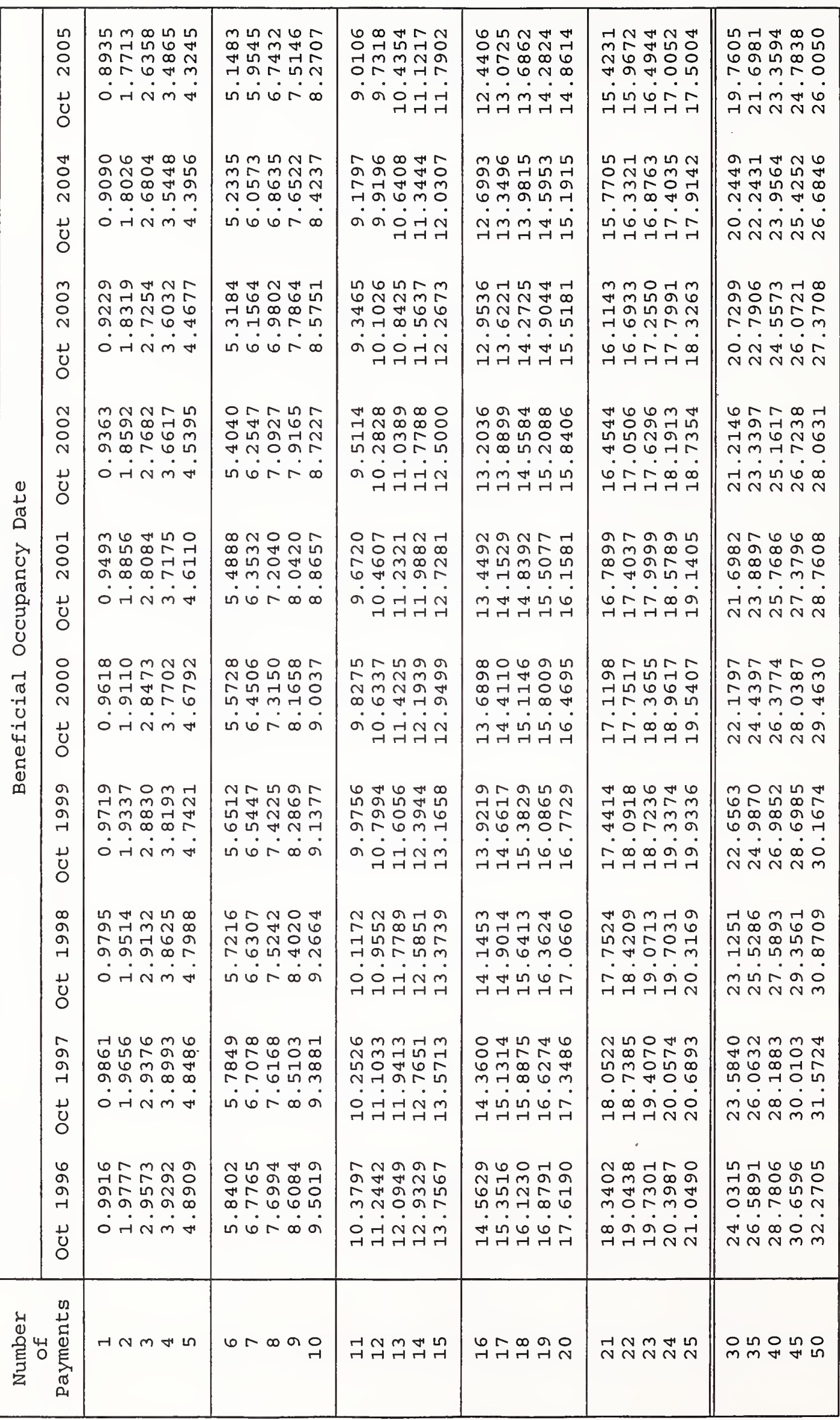

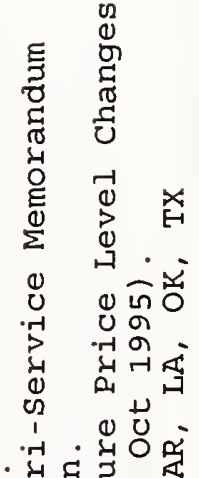

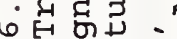
ด् ने कूष नूूष्ण ने

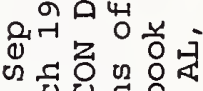
记㝴

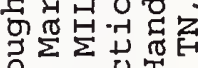

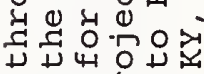

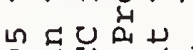
बेन्न ट द्व

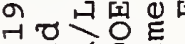
बदि U. - 0 \% ๙ू थ

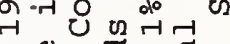
น 우유 전

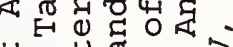

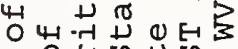

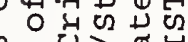

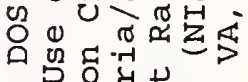
ชั E 00.700 है ข 出 $\sigma \mathrm{C}^{2} \mathrm{C}^{\mathrm{2}}$

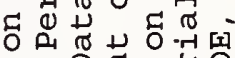
व

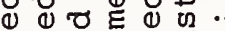
ט $N$ (1) थ б ๓

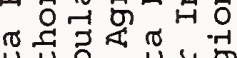
芉

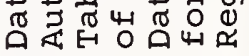
귀ำ 


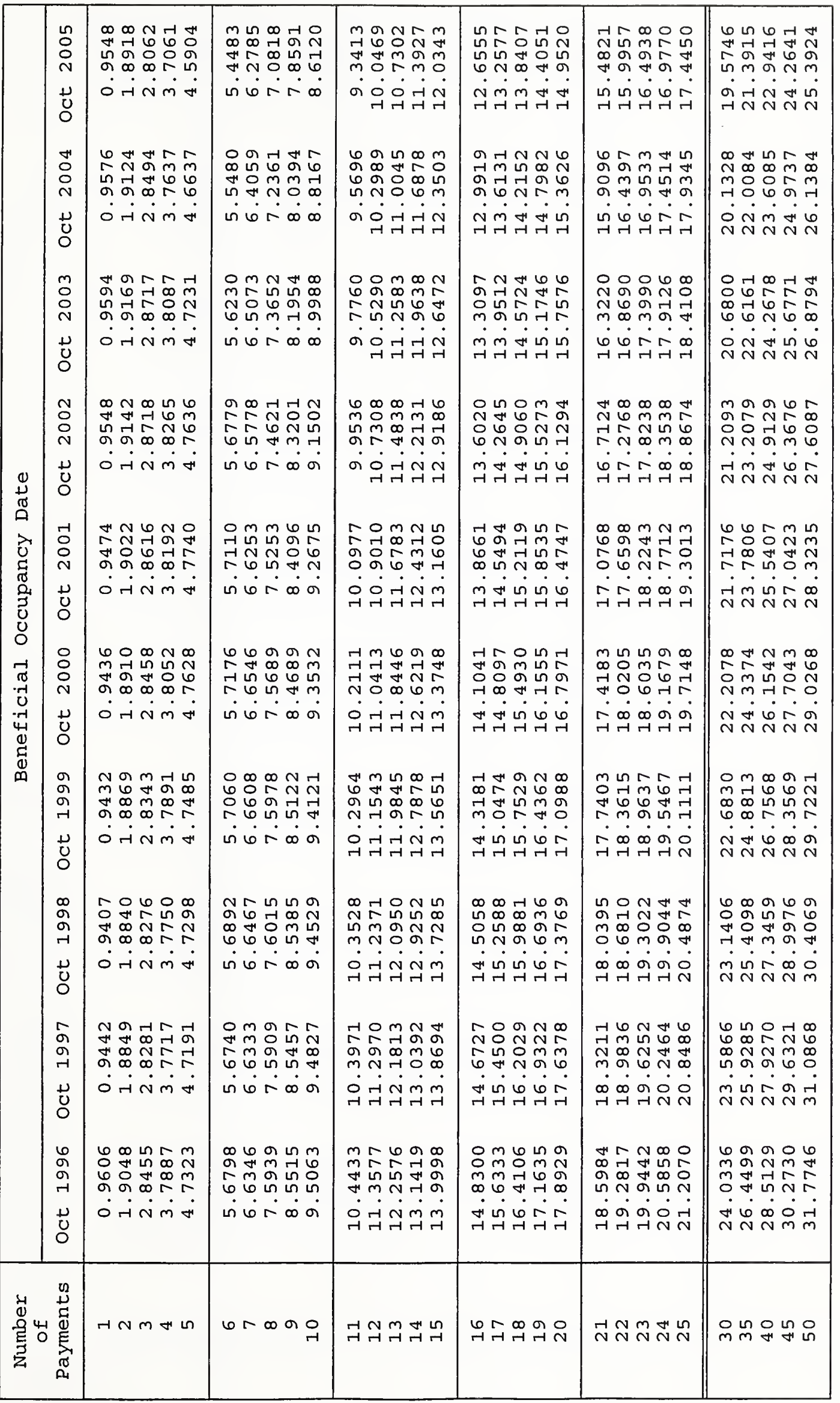

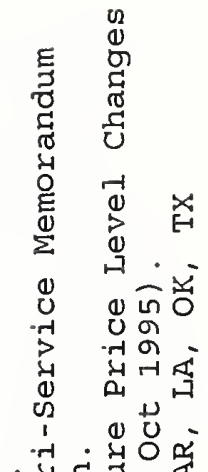

. म्य ¿ 6 स \% न बूँ बून म्न

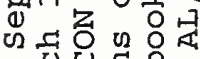
던도욤

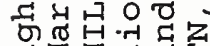

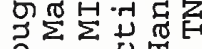

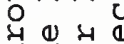

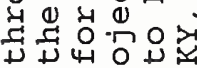
पष ๙ ने 엉을

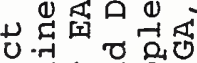

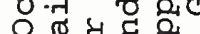
$6^{\circ}$ 艺

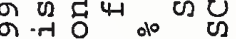
तथ $0 \stackrel{\text { ol }}{\mathrm{H}}$ मூ.

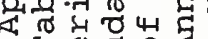
स्व

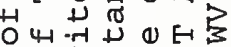
○ पु ల 只 ช

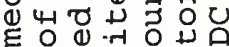

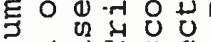
ช

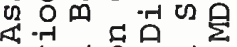
4

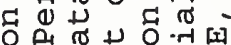

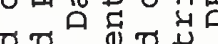

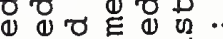
ט $N$ U थ ๘

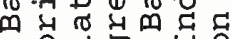
염 की

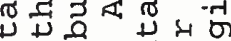
त न

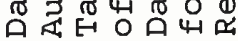
$\hat{r} \stackrel{\hat{v}}{\hat{v}} \stackrel{\hat{m}}{v}$ 


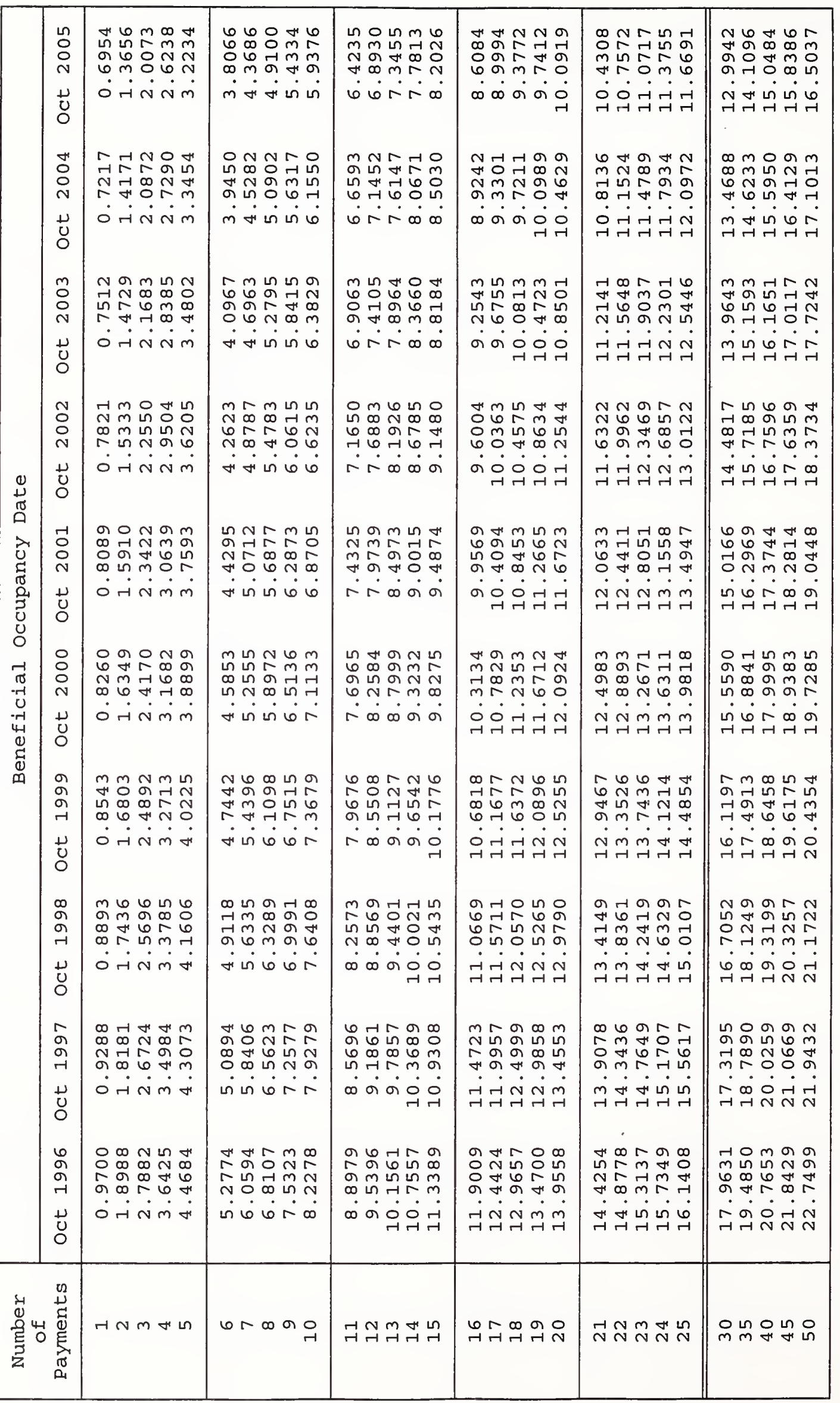

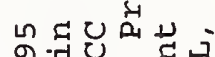

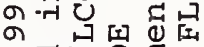
-

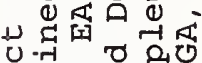
บ.न वृ ठ 6 정

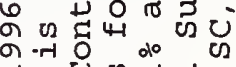
ने $\begin{array}{r}-r \\ 0\end{array}$

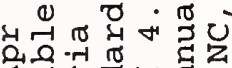

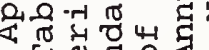

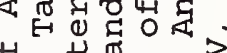

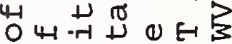

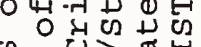
\% 虾

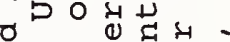

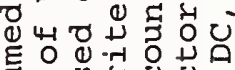
है 020 年 㧒-

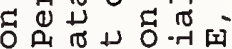

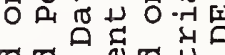
वृ बृ

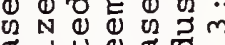

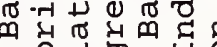
ه ரु

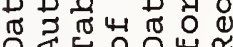
$\hat{v} \hat{v} \quad \hat{v}$ 


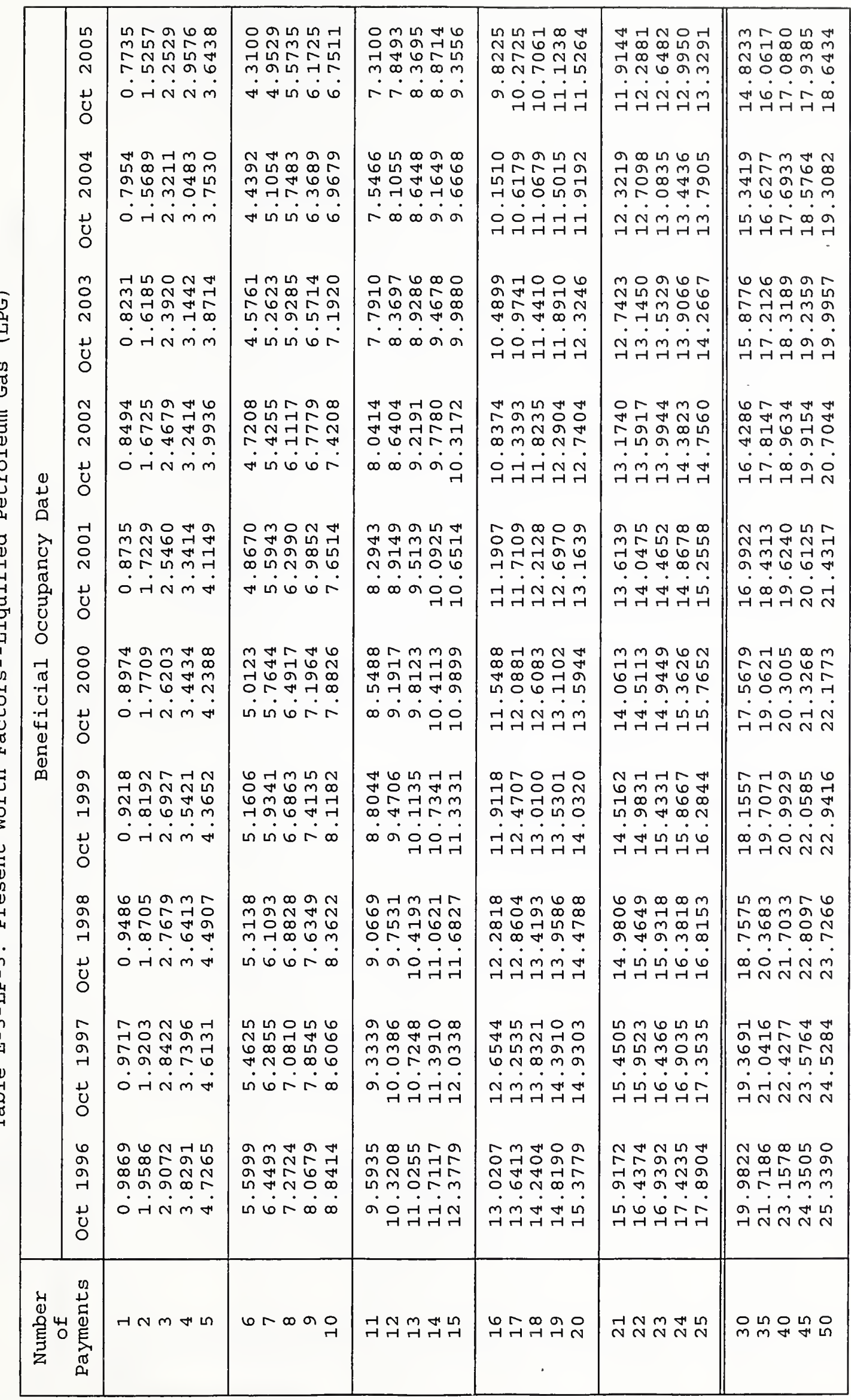

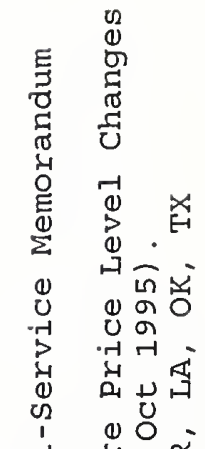

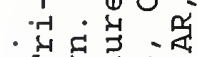
世 क्षे

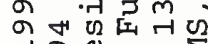
नूेष्山出

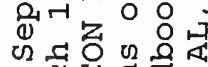
Uण द व

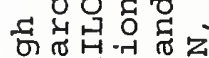

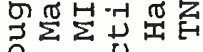

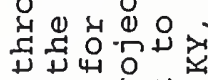
に ผू. द्न 月. 造雷 . О्ठ 6 出 बून्त द्व

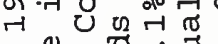
मे б

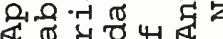

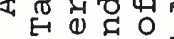

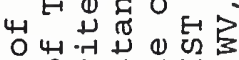
O ข 纪 ชั

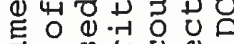

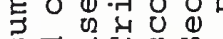

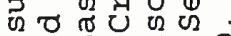

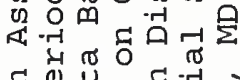

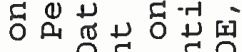
ช ช \&

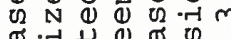

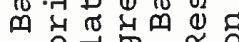
凹 ग

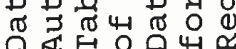
$\hat{i} \hat{v} \quad \hat{v}$ $\ddot{2}$
$\stackrel{0}{0}$
$\stackrel{0}{z}$ 


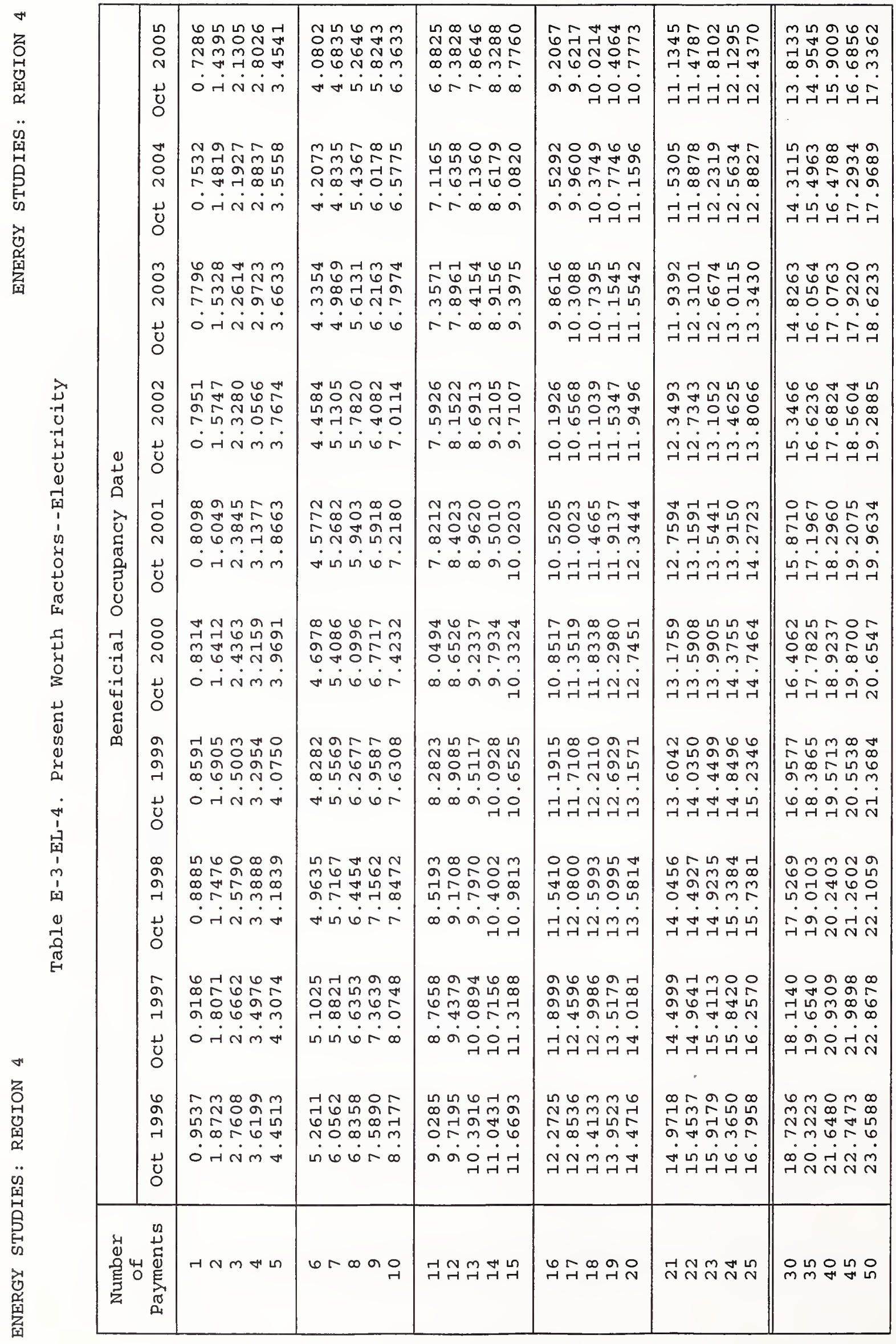

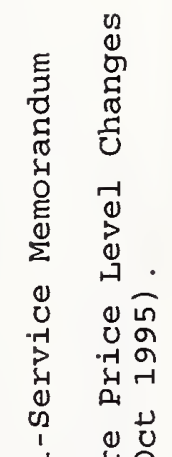

. 던 넉

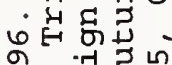

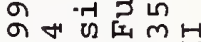

तब बैं

थल्त

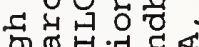

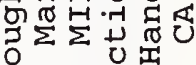

엉 4 प

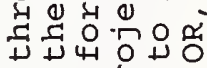

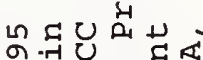

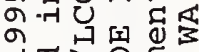
궁ํㅇㅇㅛ

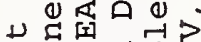
ข.न ठ을 6 बै य

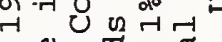
4थ

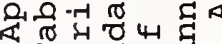
EO W 药出 ש 0 U ช

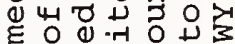

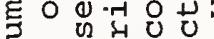
ज्व त्य थै

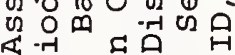
य

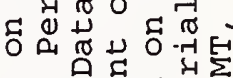
б ठ U

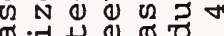

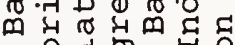
๘

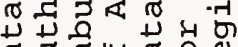

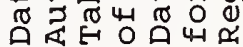
$\hat{v} \stackrel{\hat{v}}{\hat{v}}$ ĩ 


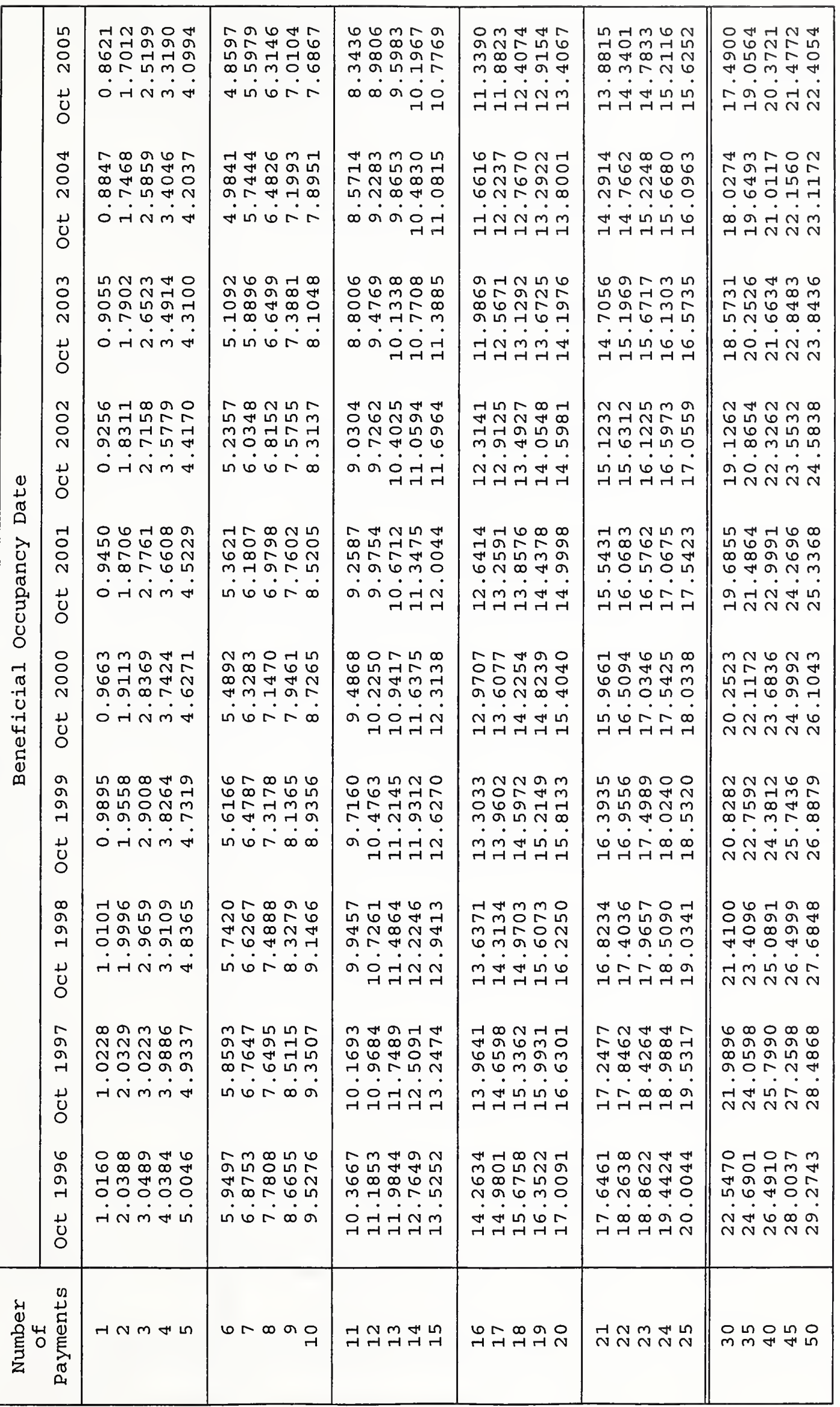

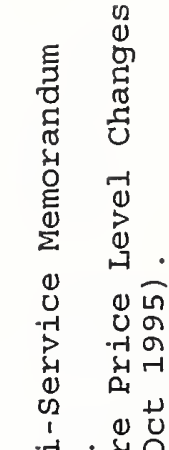

- $\dot{1}$ \& மै का बेन नुषू山्य बूँ म

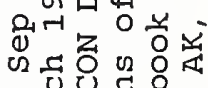
ع

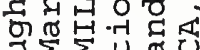

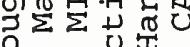

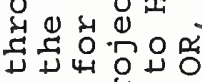

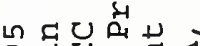
๙

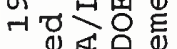

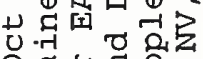
- 0 व

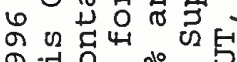

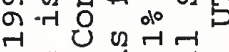
ब

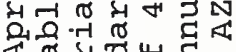

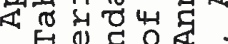
H岑苛U 0 斗次比

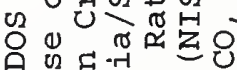
四 व

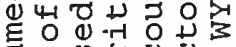

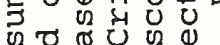
o यु

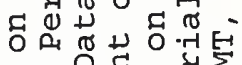
ठ व बत्ठ ह ए ज ..

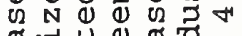
ซ ต मू न्न क्

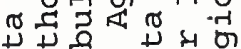

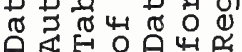
$\hat{i} \hat{v} \quad \hat{v}$ $\ddot{0}$
0
+
0
$z$ 


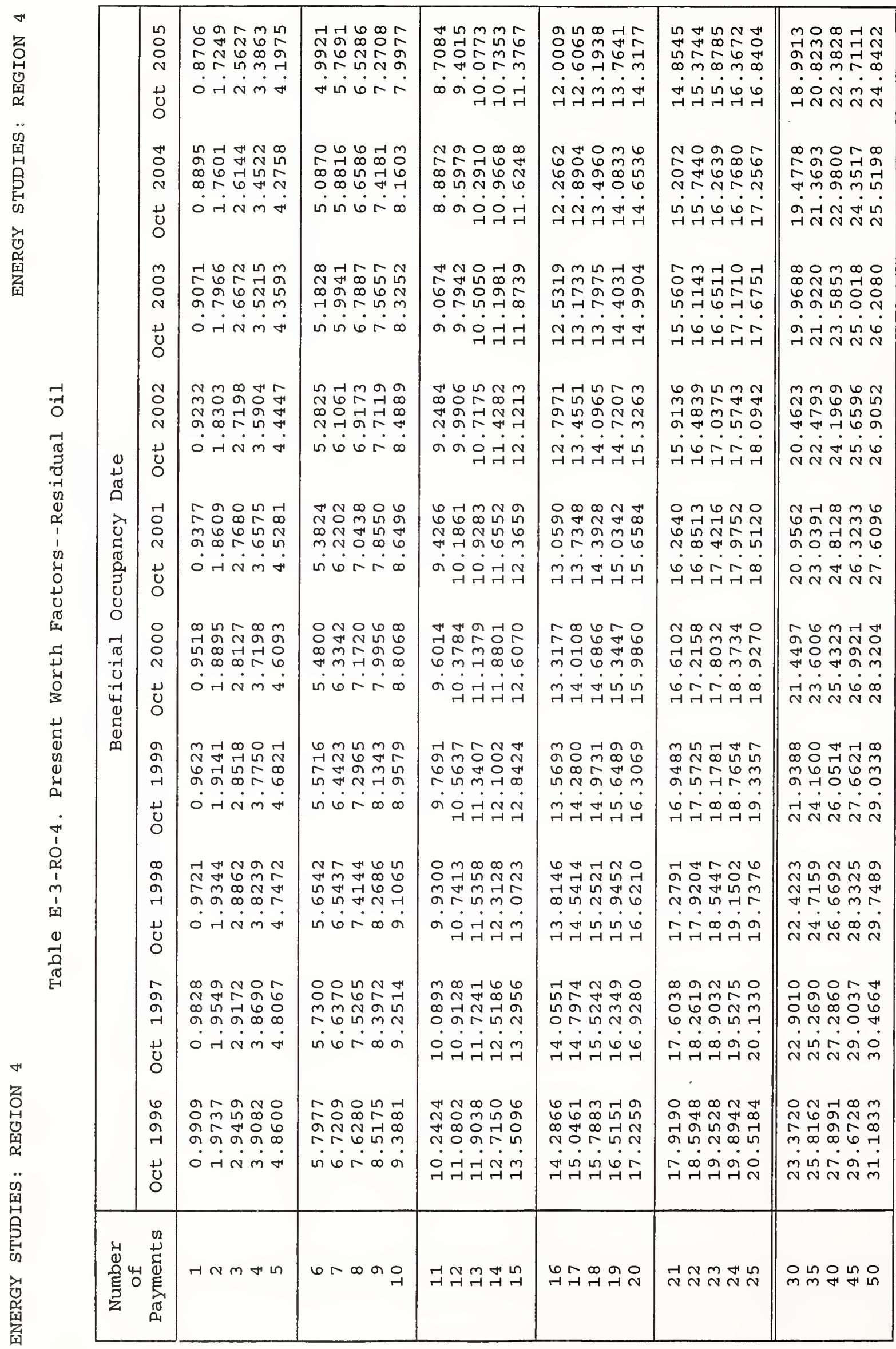

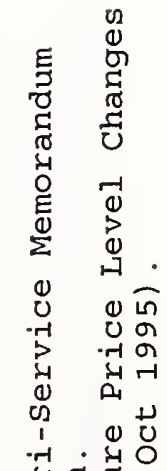

๑ั ดे ตั बै गे प्य नक्ष व्न म

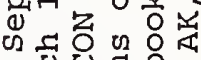
UU ⿷匚⿱一兀)

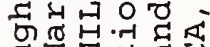

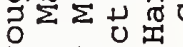
近界 เก दU मी केन्न प्र म्ञ निष्ञ⿰용 넘 . О

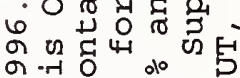
न U च 4 영요 당 ब 出出年O ○出武岕过昆 ๗ ठ

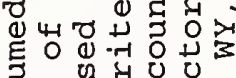
थत ข

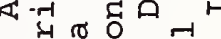
ป ه ชูष ⿰纟: N

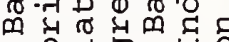
๘

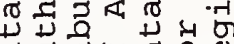
๙

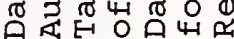
$\hat{v} \hat{v} \quad \hat{v}$ on 


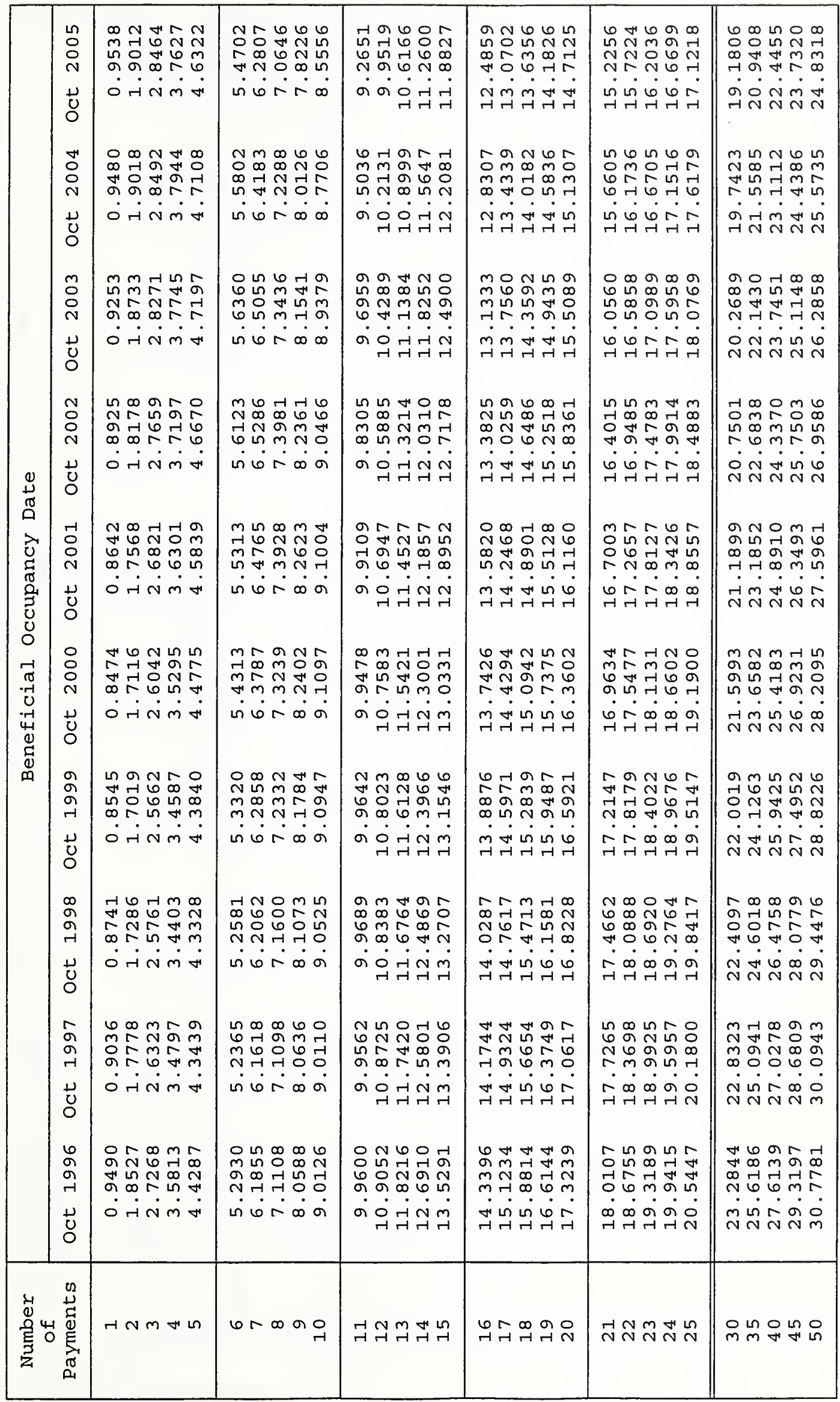

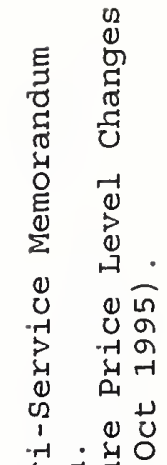

०

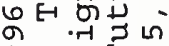
ने बूँ तब बै

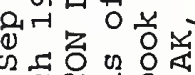
थ

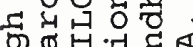

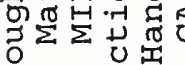

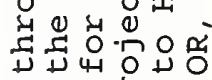
เ

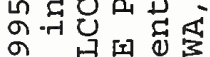
नेष回器 造质 U. - 0 \% क्ष

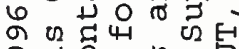
न 4 요다 त

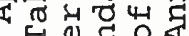
E D

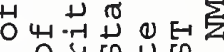
O

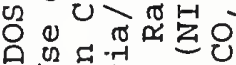

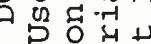
ช

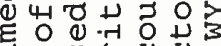
ण 约然 य.

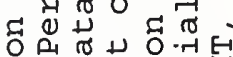
ه我艺 ช 次过记

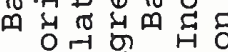

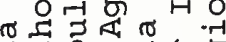
山्ل

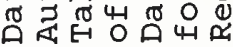
$\hat{i} \hat{v} \quad \hat{v}$ 


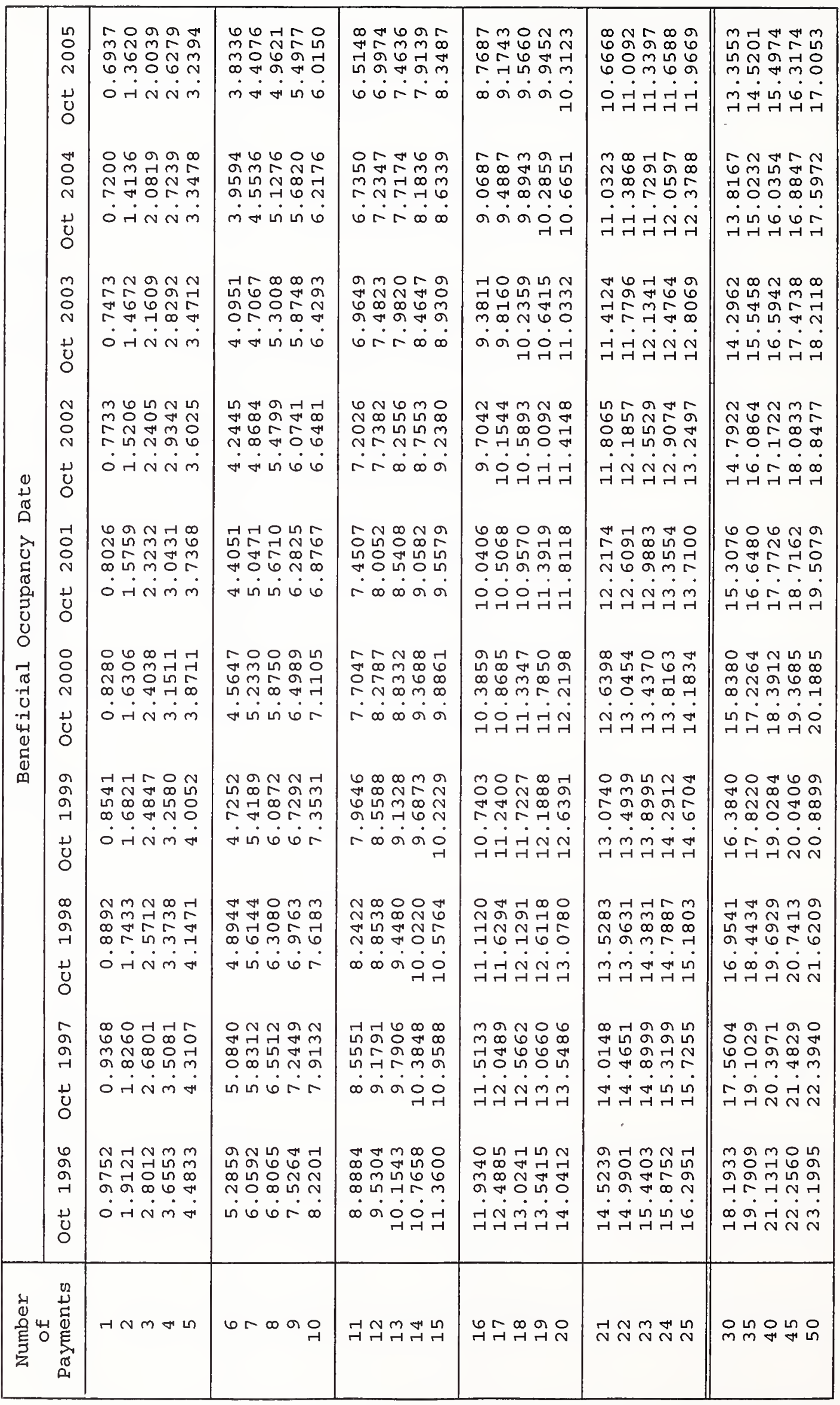

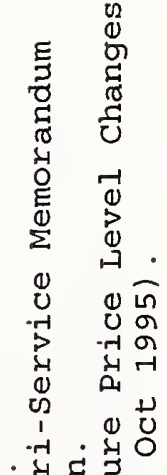

论

ूँ

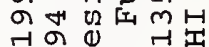

वि क्षि

थ

ठ

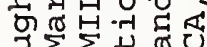

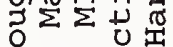

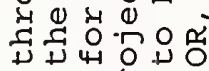

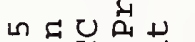

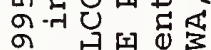

$\rightarrow 70$

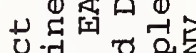

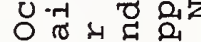

०. o. - $\delta^{\circ}$ do

4.

क्वी

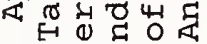

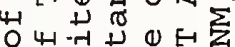

牙步焉

סै

可

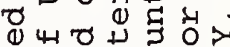

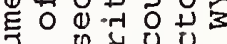

可 020

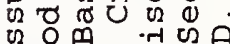

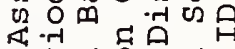

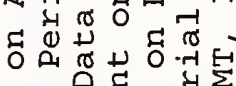
ช

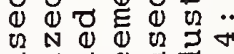
Фั-

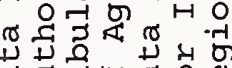

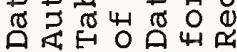

$\hat{\vec{v}} \stackrel{\hat{v}}{\hat{v}} \quad \hat{v}$

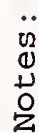




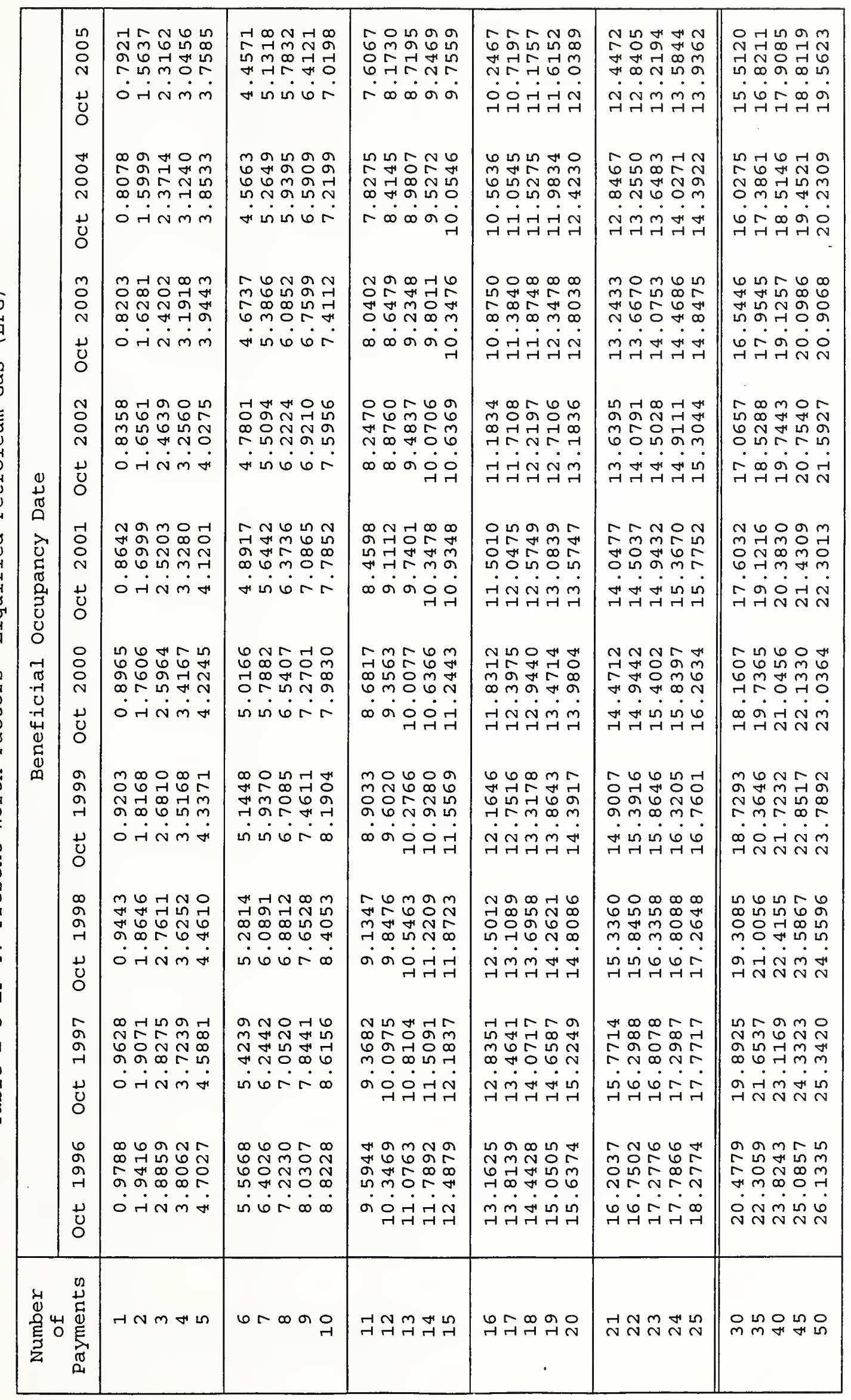

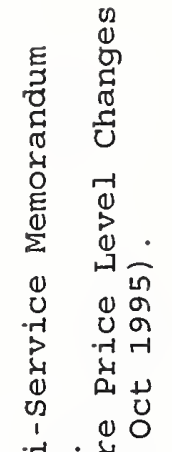

- म्ने ट्च

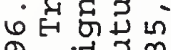

बे भण्ड

ने बूँ

مी

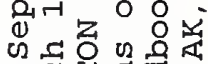

ช

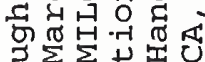

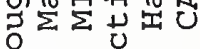

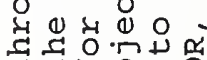
它岳品完

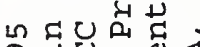
बनन्म

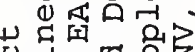
ป. - 0 क्ष द्व \%ั 岂 त्न

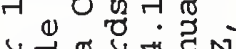

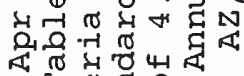
H离空

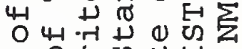
O

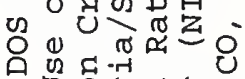

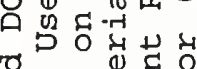

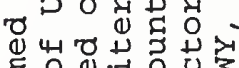

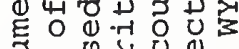

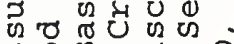
得 यु

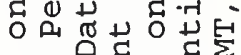
б

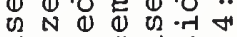
$\pi \cdot-1000$ ต प्रु ๘空 马 त క $\stackrel{\hat{v}}{\hat{v}} \hat{v}$ in 


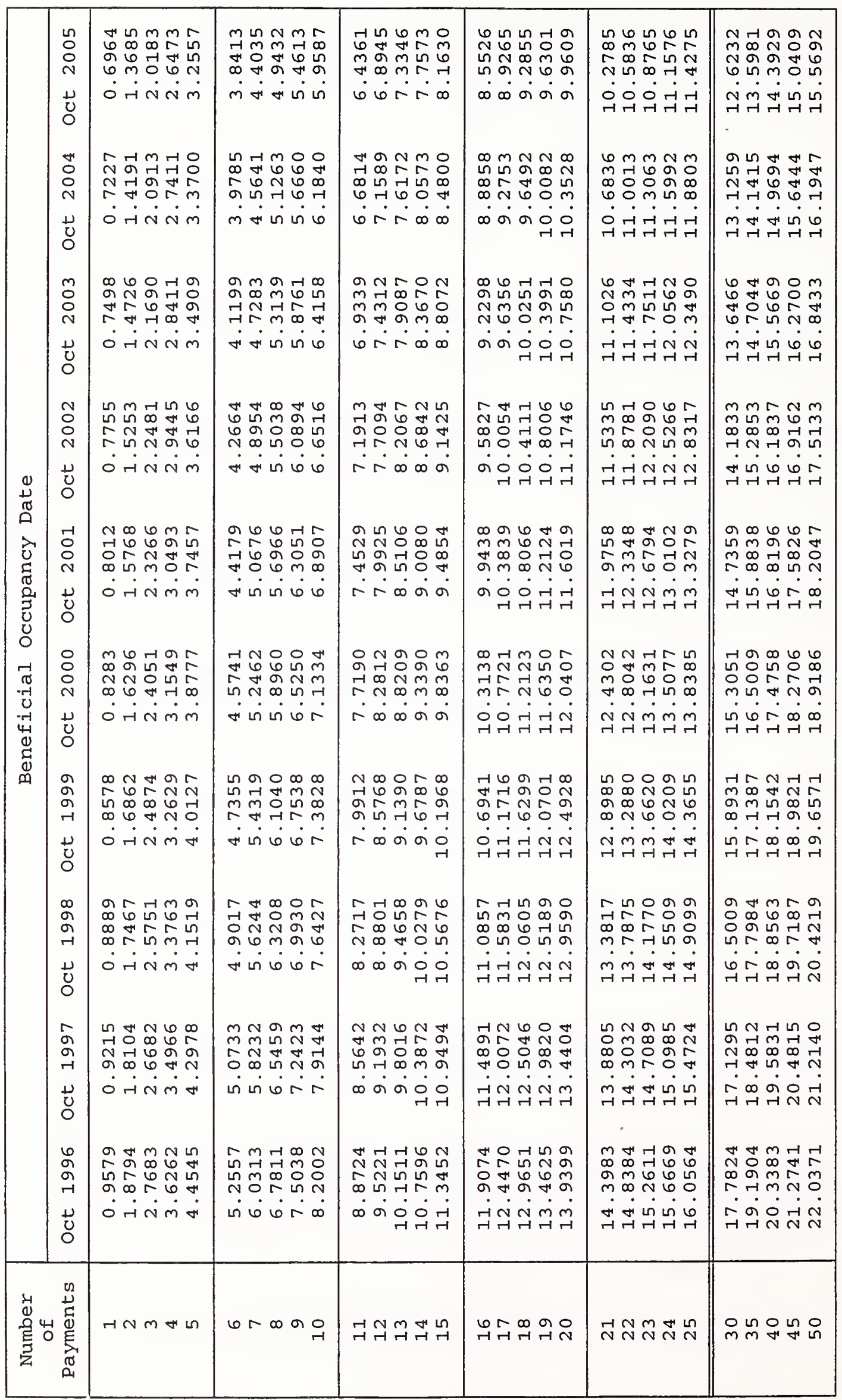

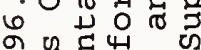

न ल ट म

न न्न 0 ग म

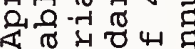

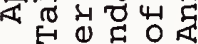
屮 O 4 - 10 の の ค ह 00 -r 0

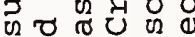
ט 0 ต ब ᄃ 5็ ه $\nabla \nabla \stackrel{1}{0}$ (1) ह ए थ U2 $N$ U O O ๓

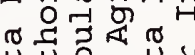
๗ 穴 


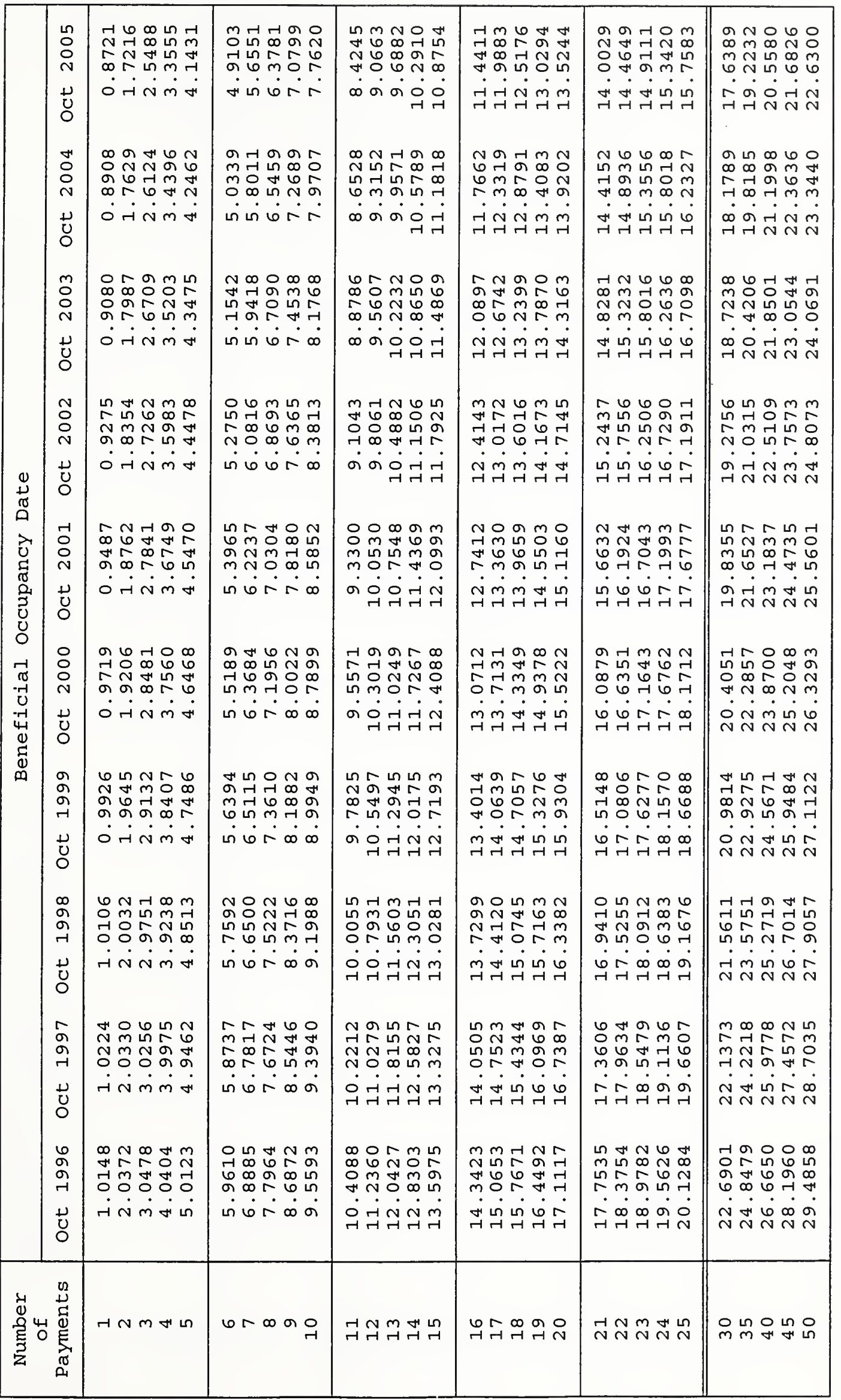

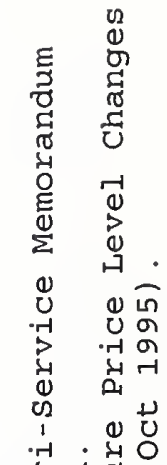

- म्र ट्

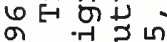

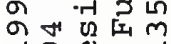
न के एँ مी न म 凶ـ 넙 号远

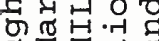
ว $\Sigma \underbrace{}_{0}$

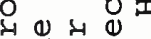

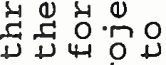
เ ब.न 다요용 बदि 岂台四实

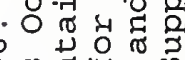
ดे थ ब.न 0 do ᄀथ 0 ⿹ 4 近需出 ED更O最

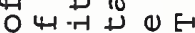
出边是 थ 细.

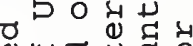
แ ○ \& ชี ข 0 ต 过药 द ठ (1)

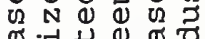
ต ० 0 品

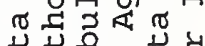

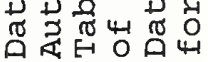
$\hat{\sim} \hat{v}$ نू 


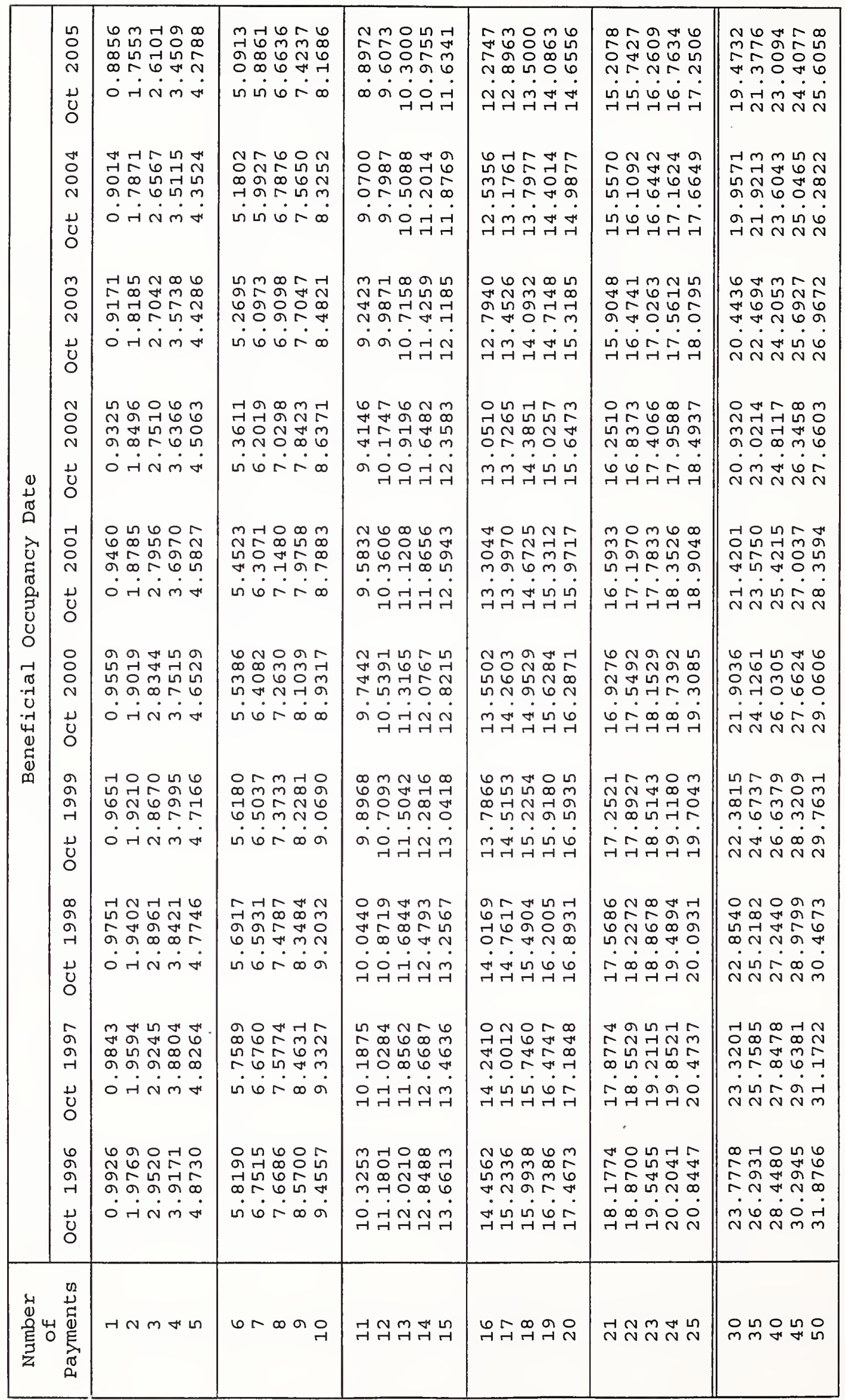

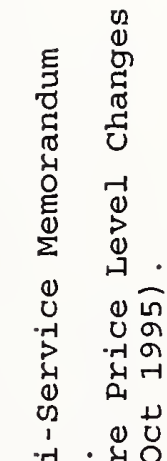

न म्न 年 ह己 ब न च

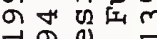
ศूั 윤낭

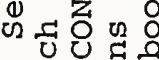
단ㄷㅁㅇㅠ 灵 $\sum^{\pi}$ O U 巧

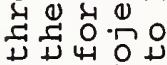
॥ ๙ू. म्न ब비 त्व 대용 近

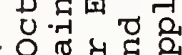
60

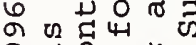

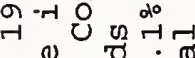
$4 \stackrel{0}{-1} \sigma$ वे

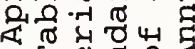

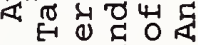

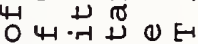
○出牙岕界思 ル 0 U

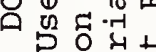
吕

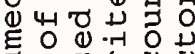

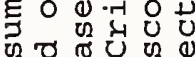
ข

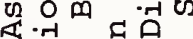
ब.

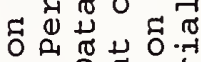
ठ थ $\mathbb{N}$ (1) ( $)$.

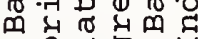
$\sigma$ 号罗

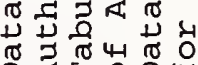
元老出出苔 $\hat{\imath} \hat{v}$ $\ddot{0}$
0
$\nu$
0
$z$ 


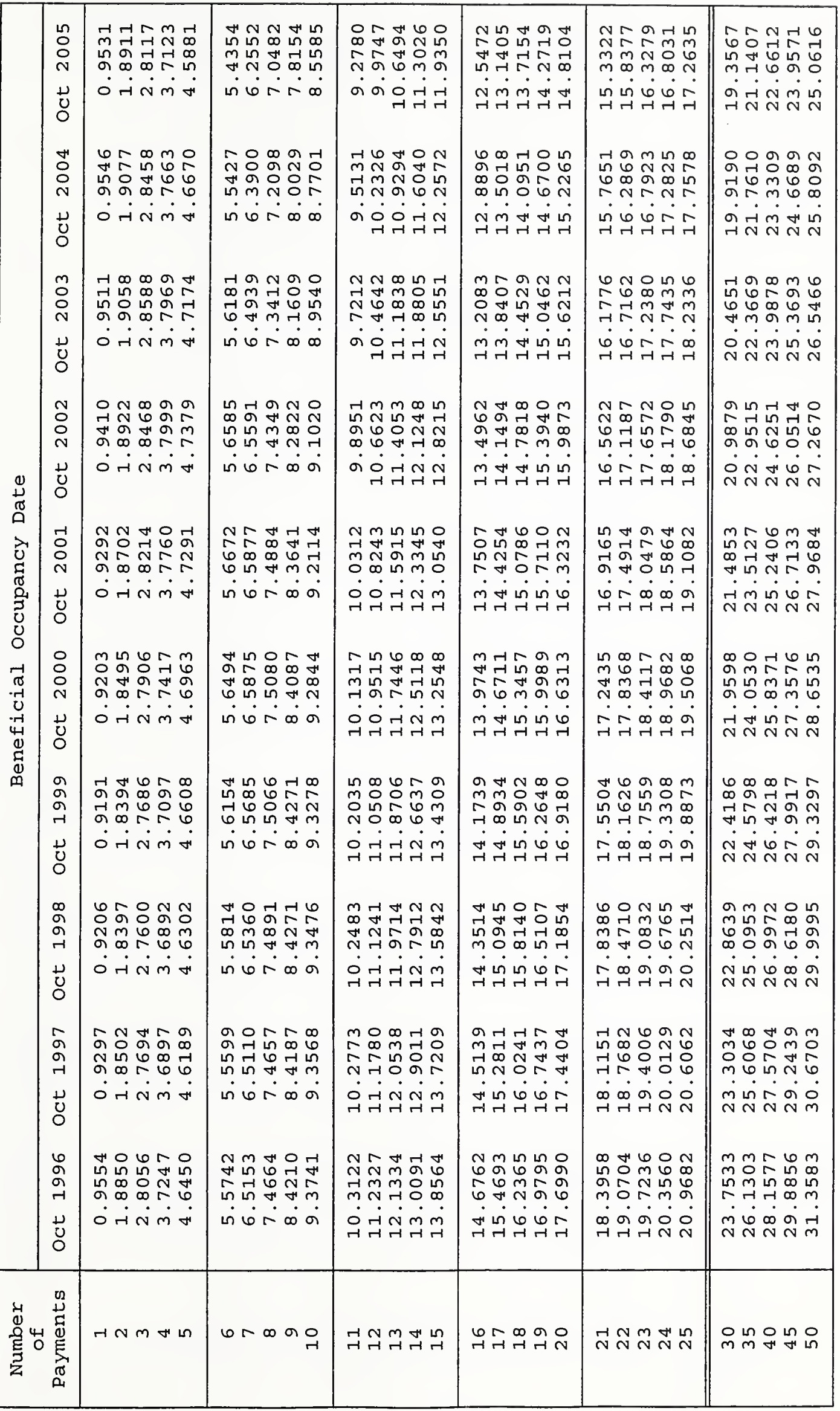

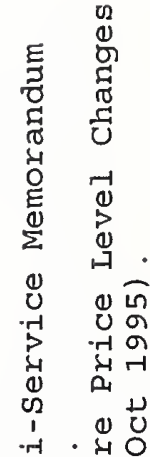

- द्र द्व

ดำ ดु

बे पूत

तबे

مू मे

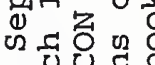

ปै

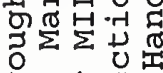

년

पम

程品 ดून्न

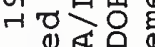
U.

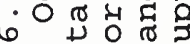
ด้ 的若 नेन 0 00 प्र.

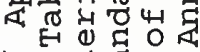

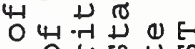
○出可出 ขण 0 员 口 శ్

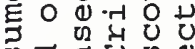
की

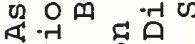
牙尔 도ㅇㅛㅠ

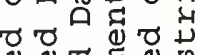
(1) \& ⿰冫 $N$ \& 0 U

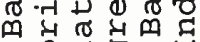
(1)

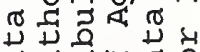

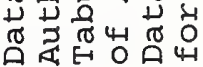
$\hat{\vec{v}} \hat{v}$

$\ddot{0}$
0
$\nu$
0
$z$ 


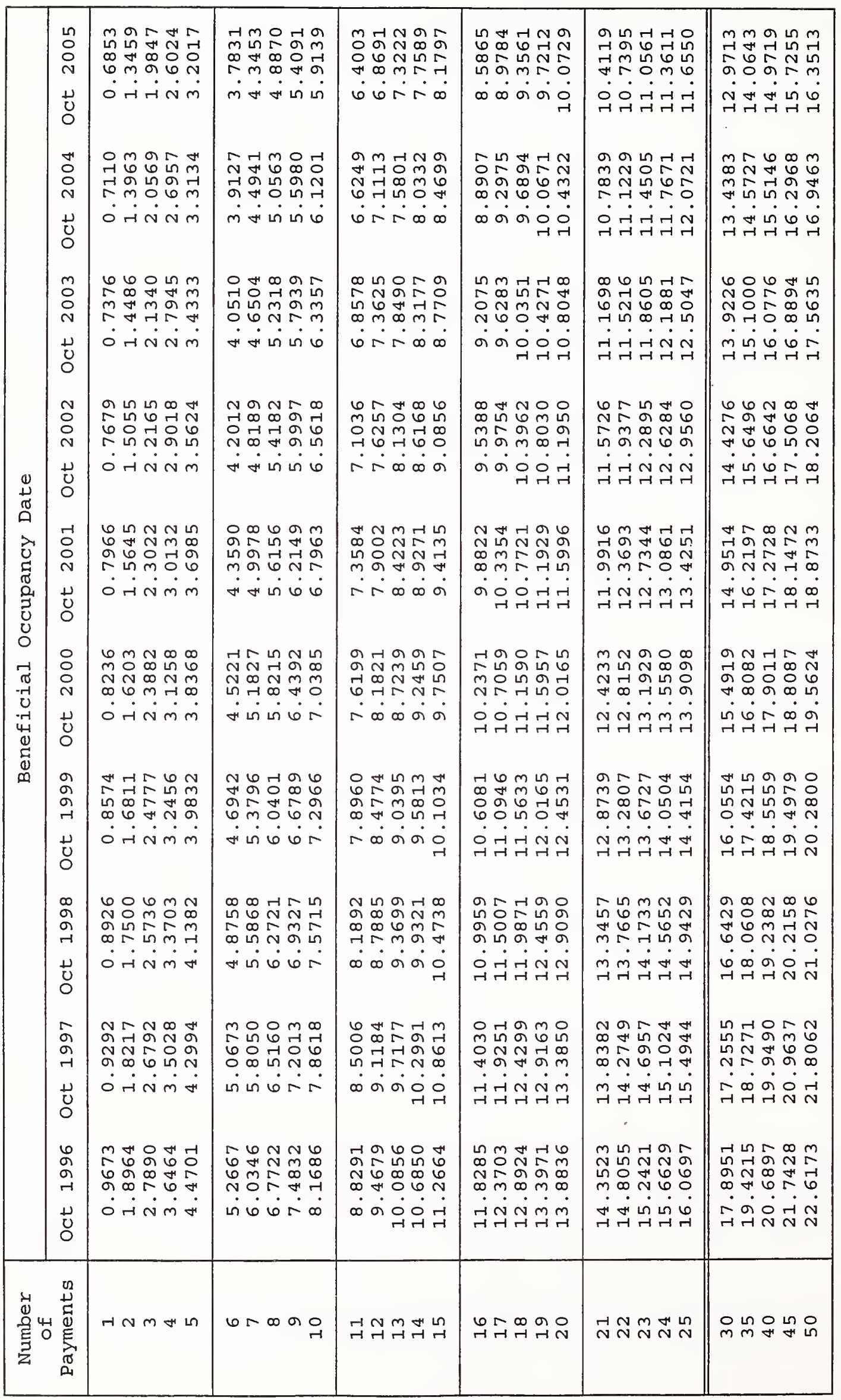

6

बर प्य

की

4

Q 0 - -1

在西要出

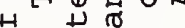

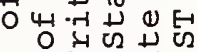

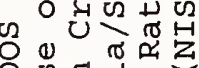

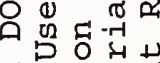

ه్

类

थ 0 क ज थ

在. 댕

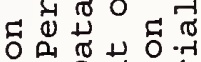
○茴茫 0 -

రृष्ठ

⿰ $N \&$ \& 2

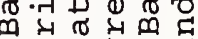

궁

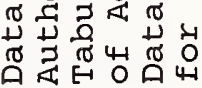

가 $\hat{v}$

$\ddot{2}$
0
$\stackrel{1}{0}$
$z$ 


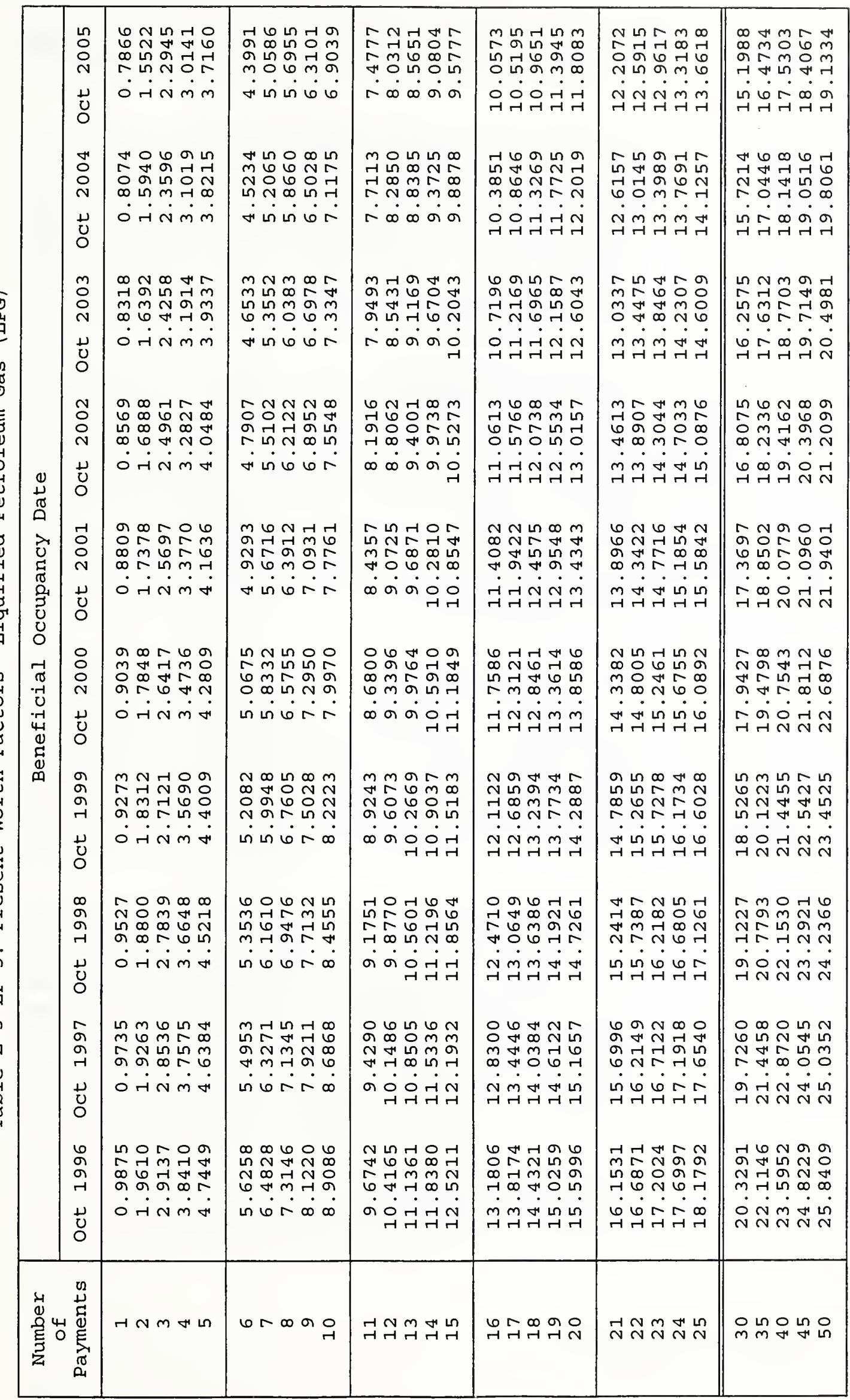

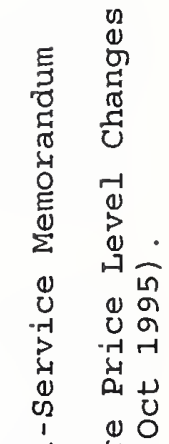

.

员

बे 可在

तूर

مิ 口岁

भून

ध

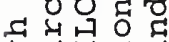

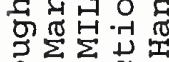

ठำ记

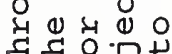

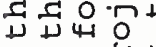

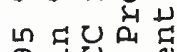

बन्न प्व

강뵈용

通口

讨年国

- 0 元 म

ถ้ थ है

केन्त

-10 0

$4 \stackrel{0}{\sigma} \pi \dot{4}$

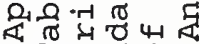

霜过岩

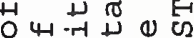

过出艺

记

万ठ क्ये है

ब4

ह 0 व

ज्ञ

20 0 m

頮

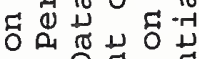

ชర

¿ \&

(1)

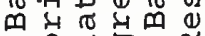

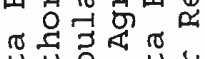

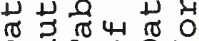

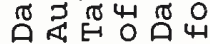

$\hat{\vec{v}} \stackrel{\hat{v}}{v}$

$\ddot{0}$ 


\section{Part II. Tables of Present Worth Factors for Non-Energy Studies}

Part II contains tables of present worth factors for use in computing the life-cycle costs of the competing alternatives in a non-energy study, where energy costs are either nonexistent or equal among all alternatives, in accordance with the provisions of governing DoD criteria (see Appendix A). The discount rates used to compute these present worth factors were taken from Appendix C of OMB Circular A-94 (January 1995). See the Preface of this report for the rationale for using a $4.6 \%$ discount rate for studies with short-term total study periods (10 years or less) and a $4.9 \%$ discount rate for studies with long-term total study periods (longer than 10 years).

Tables NE-1-1-ST, "Present Worth Factors--One-Time Costs, Zero Differential Escalation (Short Term Studies)" and NE-1-1-LT, "Present Worth Factors--One-Time Costs, Zero Differential Escalation (Long-Term Studies)" provide factors for costs which occur one time or at irregular time intervals throughout the study period and which increase at approximately the rate of general inflation (hence the term "zero differential escalation"). These costs may include construction/acquisition costs, non-annually recurring maintenance costs, major repair and replacement costs, and retention/salvage value or disposal cost. These factors are called "single present worth" (SPW) factors. The present worth of each cost occurrence is found by multiplying that cost, in Date-ofStudy (DOS) prices, by the SPW factor corresponding to the time of occurrence (years after DOS). Interpolation is encouraged for non-integer time periods.

Table NE-1-1-ST should be used for short-term LCC studies, i.e., studies with a total study period of 10 years or less. This table is based on a $4.6 \%$ (real) discount rate. Table NE-1-1-LT should be used for long-term LCC studies, i.e., studies with a total study period longer than 10 years. This table is based on a $4.9 \%$ (real) discount rate. Note that the total length of the study period determines which table to use, not the time that a one-time cost is incurred. Thus, for a non-routine maintenance cost that occurs in the fifth year after the Beneficial Occupancy Date (BOD), in a LCC analysis with 30 years of beneficial occupancy (i.e., occupancy or usage), use the 5-year factor from table NE-1-1-LT (0.7986).

Tables NE-1-2-ST, "Present Worth Factors--One-Time Costs, Non-Zero Differential Escalation (Short-Term Studies)" and NE-1-2-LT, "Present Worth Factors--One-Time Costs, Non-Zero Differential Escalation (Long-Term Studies)" provide present worth factors for costs which occur one time or at irregular intervals throughout the study period and which change faster or more slowly than general price inflation. The "differential escalation rate" is the difference between the rate of increase in the particular type of cost under consideration and general price inflation. ${ }^{1}$ Present worth factors are shown for differential escalation rates ranging from $-5 \%$ to $+5 \%$ in $1 \%$ increments. These factors are sometimes called "modified single present worth" (SPW*) factors. The present

${ }^{1}$ A more accurate way of calculating the differential escalation rate for a project-related cost is:

$\mathrm{e}=(1+\mathrm{E}) /(1+\mathrm{I})-1$

where $\mathrm{e}=$ the differential escalation rate,

$\mathrm{E}=$ the actual escalation rate, and

$I=$ the rate of general inflation. 
worth of each cost occurrence is found by multiplying that cost, in DOS prices, by the SPW* factor corresponding to the time of occurrence (years after DOS) and the differential escalation rate. Interpolation is encouraged for time periods and escalation rates other than those shown on the table.

Table NE-1-2-ST should be used for short-term LCC studies, i.e., studies with a total study period of 10 years or less. This table is based on a $4.6 \%$ (real) discount rate. Table NE-1-2-LT should be used for long-term LCC studies, i.e., studies with a total study period longer than 10 years. This table is based on a $4.9 \%$ (real) discount rate. Note that the total length of the study period determines which table to use, not the time that a one-time cost is incurred. Thus, for a non-routine maintenance cost that is expected to occur in the fifth year after BOD and is expected to increase at an average annual rate of $2 \%$ above inflation, in a LCC analysis with a 30 year beneficial occupancy period, use the 5-year factor from table NE-1-2-LT (0.8692).

Table NE-2-1 provides present worth factors for costs which are incurred annually throughout the study period, such as routine maintenance and repair costs, and which are not expected to change faster or more slowly than the rate of general inflation. These factors are called "uniform present worth" (UPW) factors. The factors in this table are based on the assumption that the DOS is in April 1996 , the BOD is in October of the same year or a future year, and that the annual cost occurs approximately at mid-year during each year of occupancy, or represents the sum of several costs distributed relatively uniformly throughout the year. The present worth of a cost recurring annually over the study period is found by multiplying the annual amount, in DOS prices, by the appropriate UPW factor. The number of payments generally corresponds to the number of years in the study period after BOD. Interpolation is encouraged for study periods and for BODs other than those shown on the tables.

Tables NE-2-2 through NE-2-11 provide present worth factors for costs which are incurred annually throughout the study period and which are expected to change faster or more slowly than the rate of general inflation. The differential rate of escalation is included in the table title; these differential rates range from $-5 \%$ to $+5 \%$ in $1 \%$ increments. Present worth factors which include escalation are sometimes called "modified uniform present worth" (UPW*) factors. The UPW* factors in this table are based on the assumption that the DOS is in April 1996, the BOD is in October of the same year or a future year, and that the annual cost occurs approximately at mid-year during each year of occupancy, or represents the sum of several costs distributed relatively uniformly throughout the year. The present worth of a cost recurring annually over the study period is found by multiplying the annual amount, in DOS prices, by the appropriate UPW* factor. The number of payments generally corresponds to the number of years in the study period after BOD. Interpolation is encouraged for study periods and escalation rates and BODs other than those shown on the tables.

As noted in the Preface, the tables for annually recurring costs (NE-2-1 through NE-2-11) are based on the assumption that the Date of Study is April of 1996, and that the BOD occurs in October of 1996 (or October of a subsequent year, through 2005). Therefore, for an LCC analysis covering a 10-year beneficial occupancy period beginning in October 1996, the total study period would be 10.5 years (April 1996 through October 2006). Accordingly, the present worth factor for beneficial occupancy periods of 10 years or longer are computed using the $4.9 \%$ discount rate for a long-term project. 


\begin{tabular}{|c|c|c|c|}
\hline 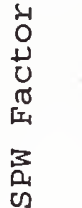 & 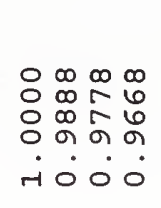 & 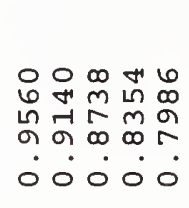 & 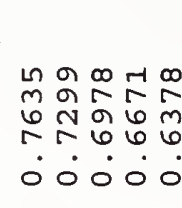 \\
\hline 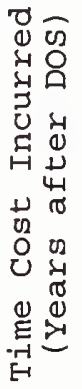 & 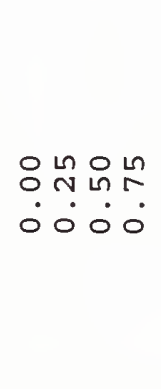 & $H N M \forall L$ & $\sigma \sim \infty \sigma o$ \\
\hline
\end{tabular}

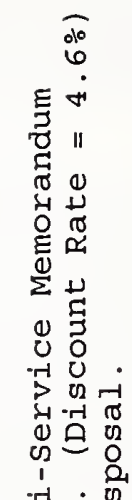

年: क

०ै है हैं

बे.

ने ूँच

ศूे ฝै

مी

๗二盯

记

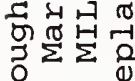

웜

뎔

ᄂ

คू. 덩

ने

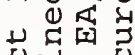

О 昆范落 - $\rightarrow$ O

(1) है ति स 10 山. 0 年的

(1) तू

记

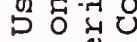

प्य ग

$00 .-1 \pi$

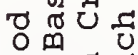

-

菏山吹

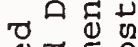

저웜

- $\perp 0$

प्र

寻是

ปี

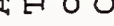

$\hat{H} \hat{v} \stackrel{\hat{v}}{\hat{m}}$

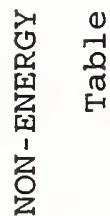

$\ddot{0}$ 


\begin{tabular}{|c|c|c|c|c|}
\hline 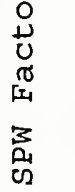 & 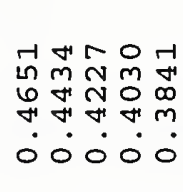 & 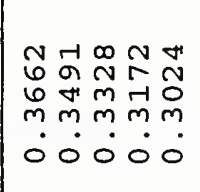 & 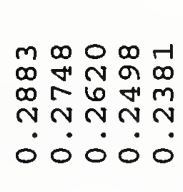 & 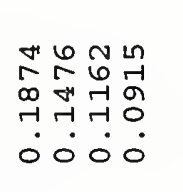 \\
\hline 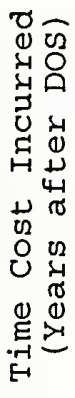 & 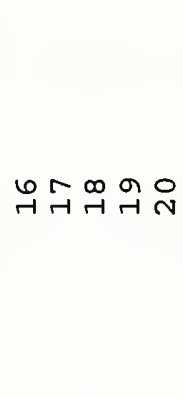 & $\stackrel{-1}{\sim} \stackrel{\sim}{\sim} \stackrel{M}{\sim} \stackrel{\sim}{\sim} \stackrel{n}{N}$ & $\stackrel{\varphi}{\sim} \stackrel{\infty}{\sim} \stackrel{\sim}{\sim} \stackrel{0}{\sim}$ & 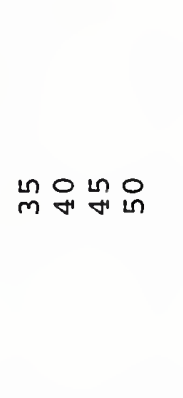 \\
\hline 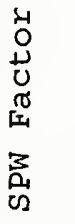 & 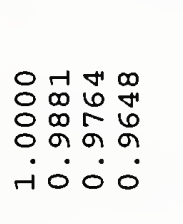 & 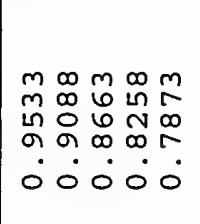 & 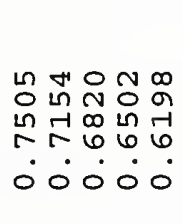 & 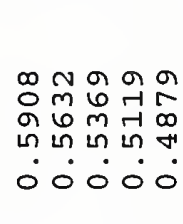 \\
\hline 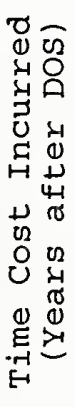 & 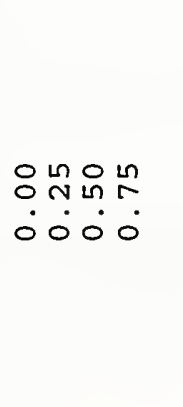 & HNMオ゙ & טr $\infty \underset{\Gamma}{0}$ & تત \\
\hline
\end{tabular}

ํำ

ह્유

ic c $^{-1}$

ถूस ज्न

Oे

तन बूँ

ब二

ठํ

ดู

亏 $\Sigma$ \%

엄 0

起

红岂

बन्न U

정

山雳雪

0

엄 40

प2 40

- $\begin{aligned} & 0 \\ & 0 \\ & 0\end{aligned}$

O.

स 0

म

प्र

U 0

ถ

एू द्व

出可䒠

$0 \underset{0}{0} \cdot \pi$

ช

-

幽

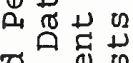

ชซ

N 0

$-\rightarrow \perp$

$4 \pi$ 设

곰원

可山

$\hat{H} \hat{v} \hat{m}$

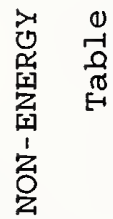

in 


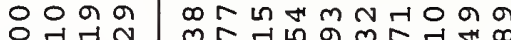
000 ㅇनत त

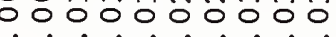

$06 \pi r$ 응 ڤ̆ ० न व ति 0 0

$\circ \sim m$ n - $6 \mathrm{~N}$ ब .. तं००००

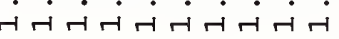

m6のm6न ᄂ H

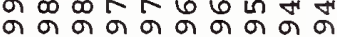
๙ न बの ब न 0000000000

$\infty$ 西 ๙ ब ब

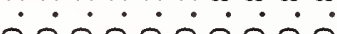

은 $\mathrm{m}$ $0 \%$

ก

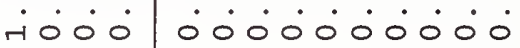

tamnN $\infty$ L $6 m$ m

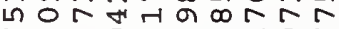
느의

○m - $n \pi$ H n

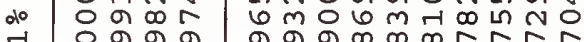

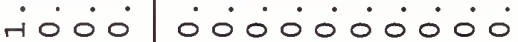

읨

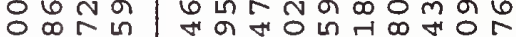

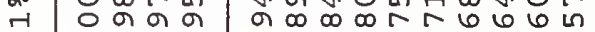

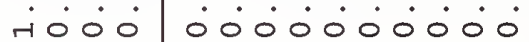

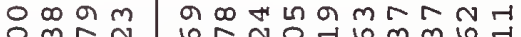
政N ․ ठबन ब

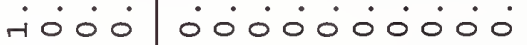

m이 mo n L $\infty \circ \infty a n m$ ㄱm

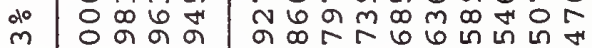
तं ○ं0்

$\circ \infty 01$ $0 \infty \infty$ 응 ति 00

$\infty m+\min n$ L

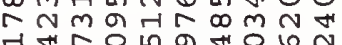

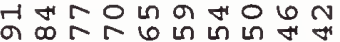
○ं $0 \dot{0} \dot{0} 00000$

nom nantonnoma

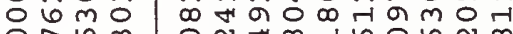

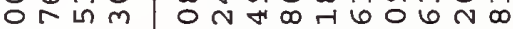
In वबन ब

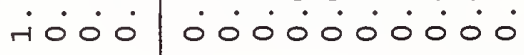

\begin{tabular}{|c|c|c|c|}
\hline & 1 & i० $0 \dot{0}$ & $\dot{0} \dot{0} \dot{0} \dot{0} \dot{0} \dot{0} \dot{0} \dot{0}$ \\
\hline 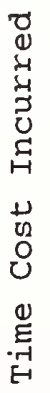 & 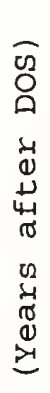 & 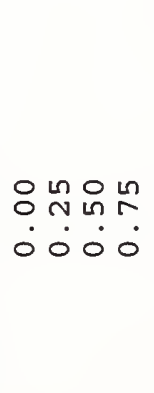 & 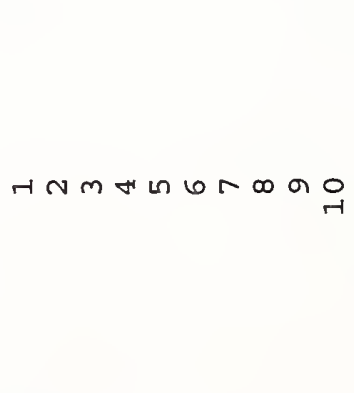 \\
\hline
\end{tabular}

ठै हैं

बे कर तब बै बै 1 बiा $z$ ज द 0

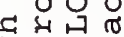
马祭㫮 ○之步

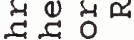
古吉出 ก ब.न त ช 兄鬼占 บ न 궁요 -

() ขै ह

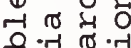

क्ष

स

山.

U

ป

○

4

$00 .-10$

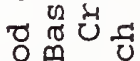

○

-

0

口㟔岂岂

ช

N

प्त्र

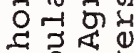

운

层 HOU

$\hat{v} \hat{v} \quad \hat{v}$

$\ddot{0}$ 


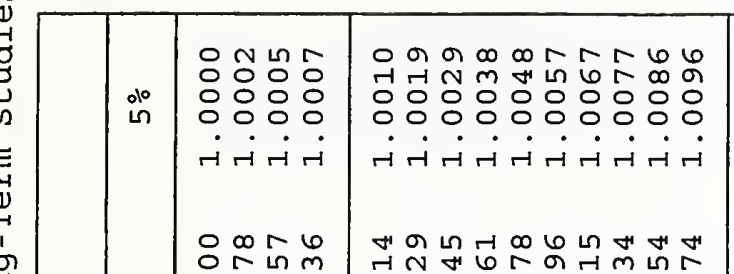

d० 응

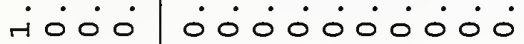

०

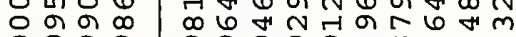

मे ठन न ब न न न न न $\infty \infty_{\infty} \infty \infty_{\infty}$

iि 0 0 0 0

द्:

$\stackrel{-1}{+2}$

每

ט

[I]

$-\pi$

(1)

음 更

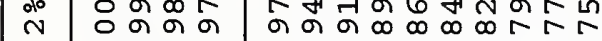

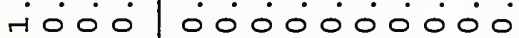

E $\circ \in \mathrm{NO}$ ते ०ून ब तं 000

은 는

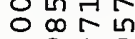

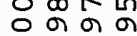
तं००००

$0 \pi \varphi m \quad n \infty \pi n \in \infty \pi N H$

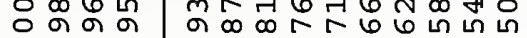
i 000 0 0000000000

$\circ 660$

$\infty 0,44 \pi 0 i \pi, 4$

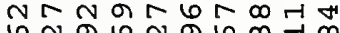

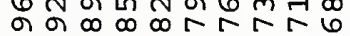

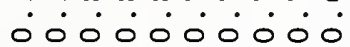

कr $6 \pi-6 \infty m a$ L $m \circ 0 m \infty 669 m \circ$ ส゙の $\dot{0} \dot{0} \cdot \dot{0} \cdot \dot{0}$

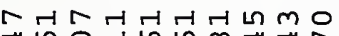
픙요 ๙ ○000000000 त०००

$\circ-16 r$

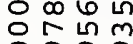

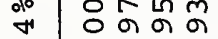
तं 000 $\circ$ in $6 \mathrm{~m}$ 응ำ

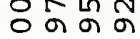
तi००

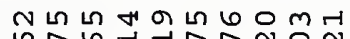
तु નூ ○ं0 $0 \dot{0} 0 \dot{0} 0 \dot{0}$

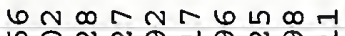

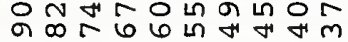
○ं0 0 0000 00 เกำ $N$ ก

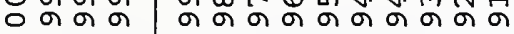
ำ 잉묘

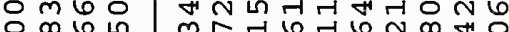

约约

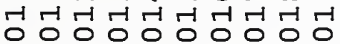

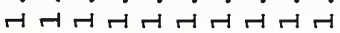

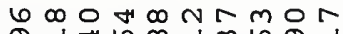
0. 年 ○0 000000000

a

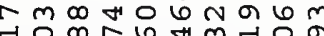

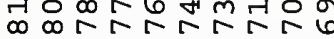
○ं $\dot{0} 0 \dot{0} 0 \dot{0} 0 \dot{0}$

6m सं

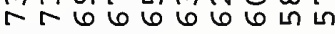

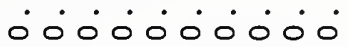

Nก A मे ஸூ 000000000

NN대

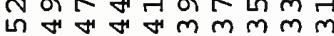
○ 000000000

40のळ4 $m \sim N$ L $06 \pi m$ M N ฟ ○ं $\dot{0} 0 \dot{0} 0 \dot{0} 0 \dot{0}$

CDAnOrnma N 0 개 N ำ m Mm N N N N N ○ं $\dot{0} 0 \dot{0} 0 \dot{0} 0 \dot{0}$

- 4 -

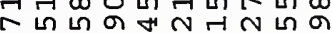

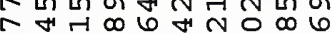
mm $m \stackrel{N}{N} \stackrel{N}{N} N$ N ०00000000.

तभा 6 \# $m 0 \Omega \forall n o w 6$ in m $\mathrm{m} N \mathrm{~N}$ N $\mathrm{N}$ त ○ं0 0000000 OกNM

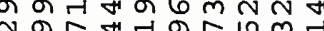

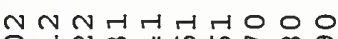
O O O O O O O O O न

I $\mathrm{mNNNm}$ H Hromban MNNHO O का $\infty \infty \infty \infty \infty \pi R T$

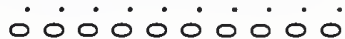

तथळのNR न1 $\infty$ सं 066666 เ

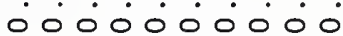

on $m N \forall-1 H \operatorname{m} m$

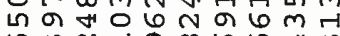

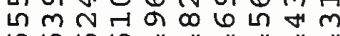

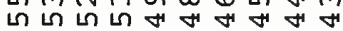
○ 0000000000

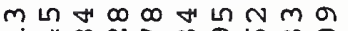
न

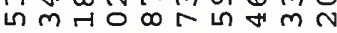
ॠ स ○ं 000000000

แก $\infty$ HN

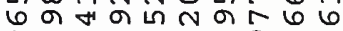

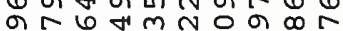
ก ก N N N N ○ं0 0000000

$6 \infty$ 개 बल เा mNO का N N N $\mathrm{NH} H \mathrm{CH}$ ○ 0000000000

N 6 NN $m \infty$ แn $\mathrm{m}$ 음 $\mathrm{m}$ बर 6 เी $\mathrm{m} N \mathrm{H}$ \% નન ○ 0 O 00000000

An n-tonm HO H

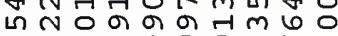
जै अ न्न

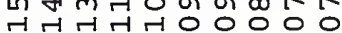
000000000

4nnnmenner N $\rightarrow 0 \%$ o 06 in तH ○ं0 0000000

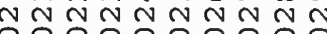

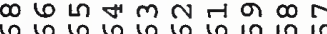

$\sigma \infty \infty \infty$ $m \infty m \infty$ $m m H^{\prime \prime}$ 0000 तं

6 เก 6 ○ $\sigma \infty \infty \circ$ m은 ○ं 000

समma 당ㅇ ก๊ 0000

o $\infty N$ ग m $N \stackrel{N}{N}$ ०००००

L) जी की तु N 0000

or $0 \mathrm{~m}$ -1 $\infty m$ เ mㅇํㅇ $\circ 0^{\circ} 00$

$\forall \infty \infty \mathrm{m}$ $\mathrm{N}$ เ $6 \mathrm{~m}$ बढ़ $\circ 000$

เก 6 เก * ma 0
0 $\circ \dot{0} \circ$

$a \infty$ 느 $\forall \infty \infty$ स당 $\circ 000$

더우 6 우 m강 응등응

$\circ 000$

वैं

-- 0

$?$ थ

मै त

๗

$\begin{array}{cc}-1 & 0 \\ -1 & 0\end{array}$

- Н्

6ै सु

बे से

तबे

م

बैद है

UU

돈동

올 봉

मे ه म

प्दी

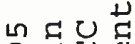

बन्न

ने

(1)

$\mathrm{O} \cdot$

0 \% म 0

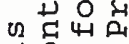

- 0

(1) 0 ठ

욤 $\pi$

때엉

$+\pi$

㟧

(ब)

凤 $=-\pi$

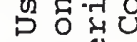

山 뽕

०

तथ

品

भु

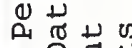

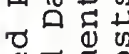

ष

N- $\perp$

मे 0 \&

억영

은

毛出
응 ㄴํㄴㅇํㄴ 는 Hnm

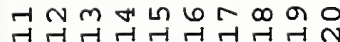

.

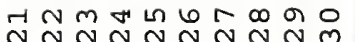

0000

느의 능요 $\hat{n} \hat{N}$ ํํ

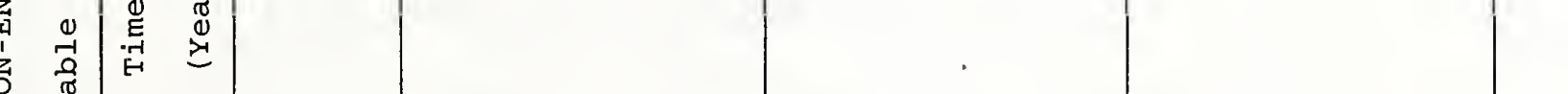




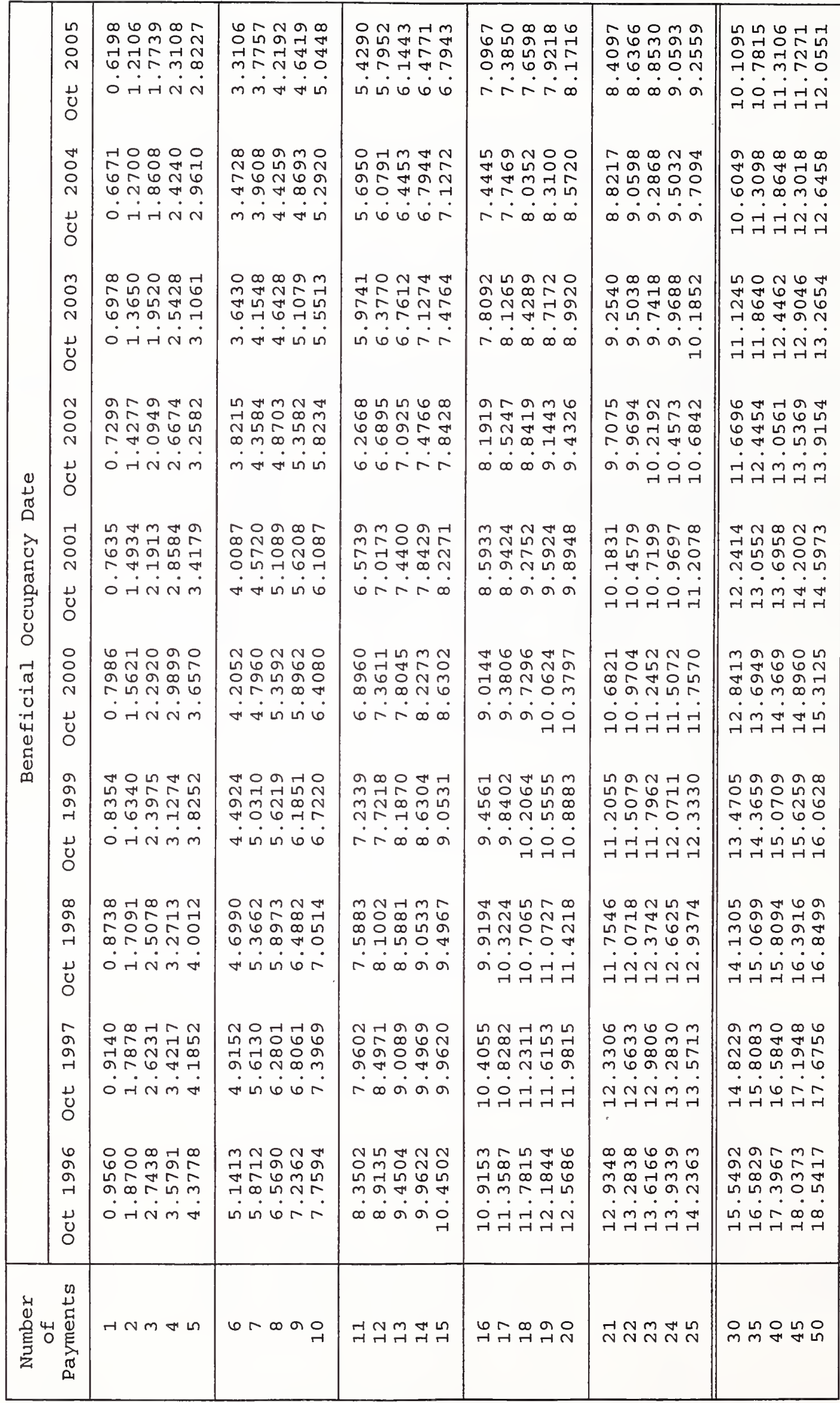

बे 24 प

बन्त ठ थ

मू.

ब

世

०4

य ठ ते

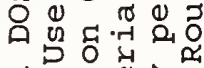

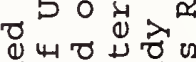

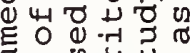

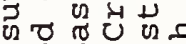

थ $\bigcirc$

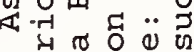

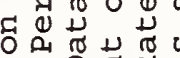

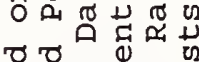

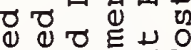

U

ब.

म न ब न 凹ั $\hat{i} \stackrel{\hat{v}}{\hat{v}} \hat{v}$ 


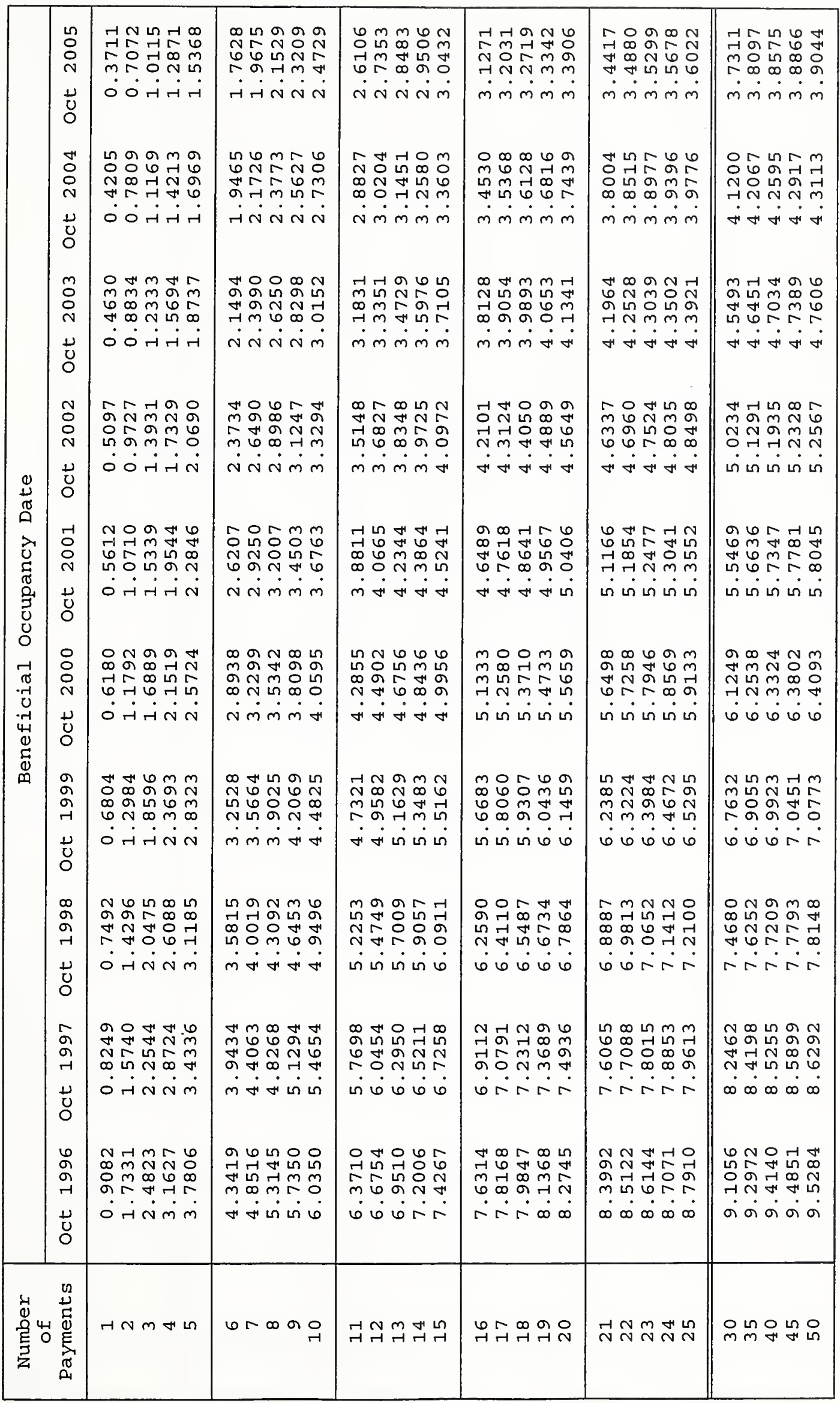

-1 ம ब ने कौ ๆ वने थ द 4 प्र

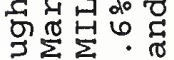
ठ $\sum \sum \dot{\pi}$

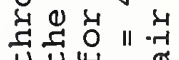

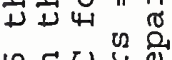
ᄂ E बन्न प्त †包这的 능 . 0 -

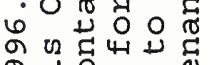

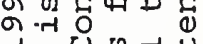
न 0 चु 4 全全年

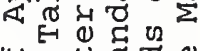

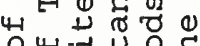

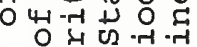
क ण प्र 吕 D 0 .

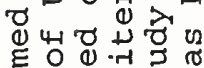
है

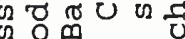

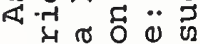
दू 0 ○范茞証 ठ ब 我 近乐近 0 ๙ 专品?

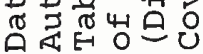
$\hat{\vec{v}} \hat{v} \quad \hat{v}$ 


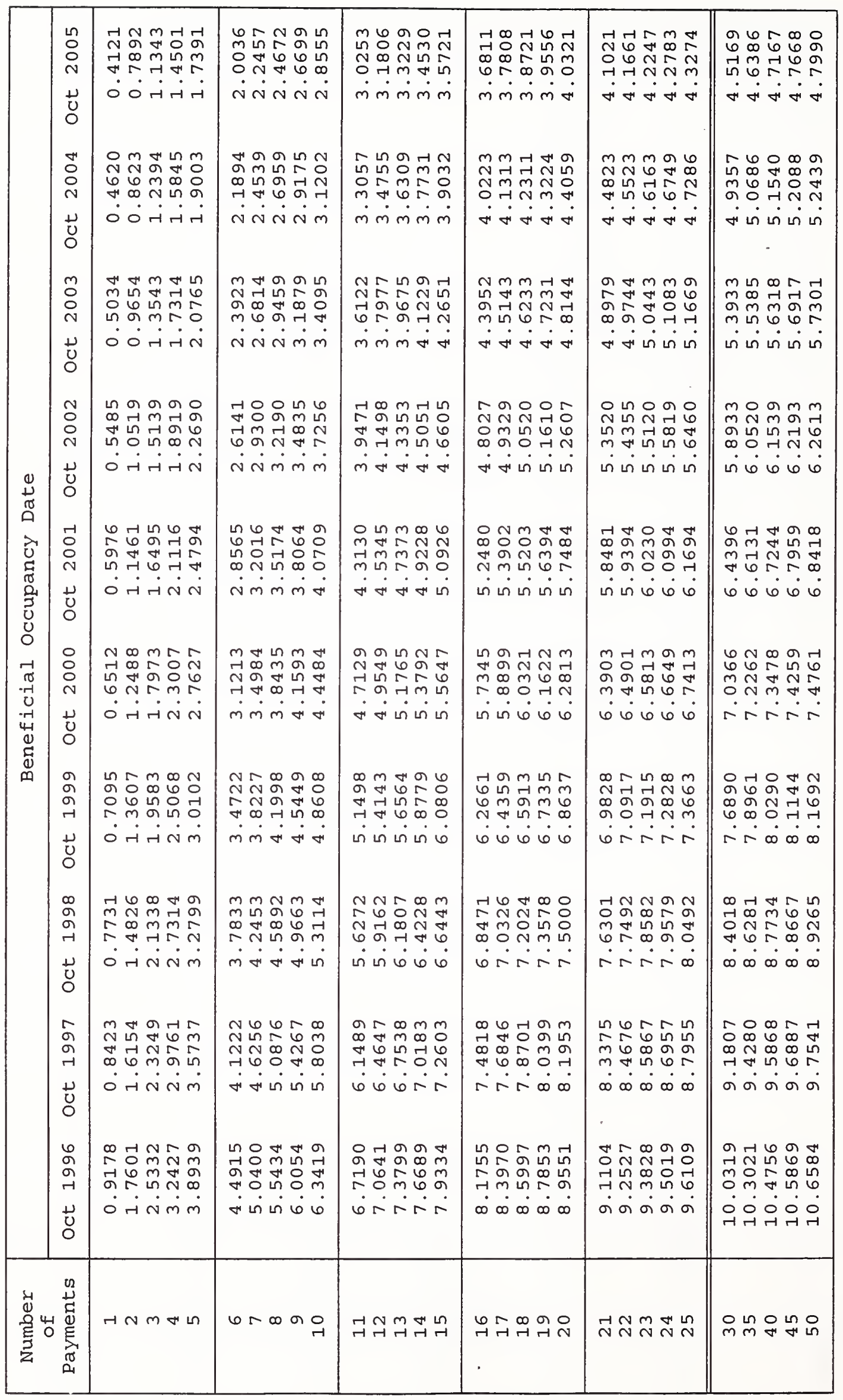

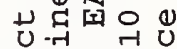
. 0 व 6 I 00 \% की तथ चु प्र 0 क्ष 0. 00 स फ

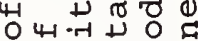
० यू 0ून्त्र थ ण

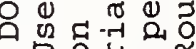

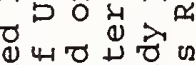

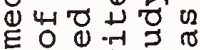

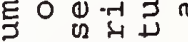

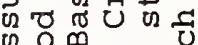
Q 《न्त ธิ 同幽 ช ซ व U $N \&$ U

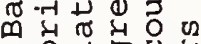
प एद 3 \& 0

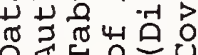
$\hat{v} \hat{v} \quad \hat{v}$ 


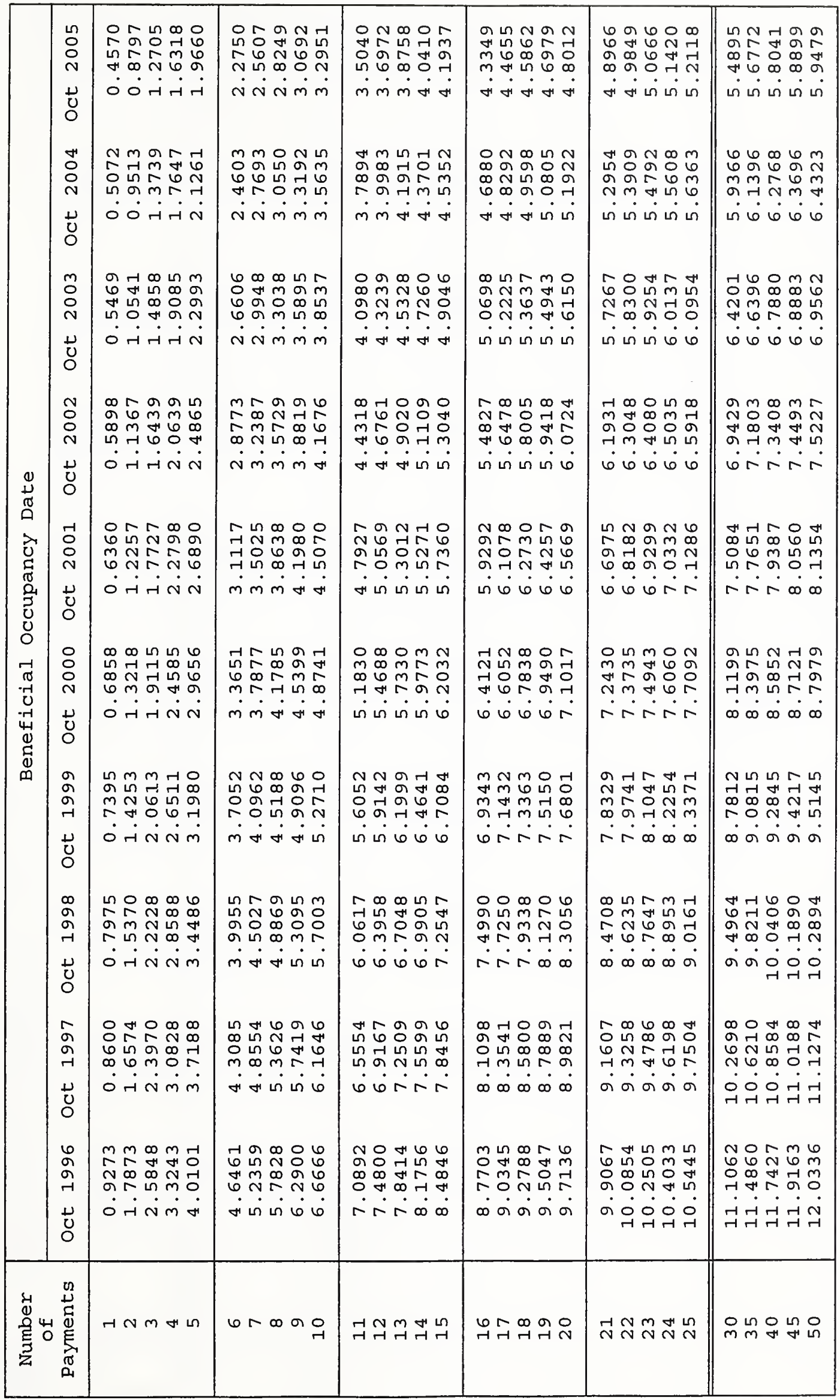




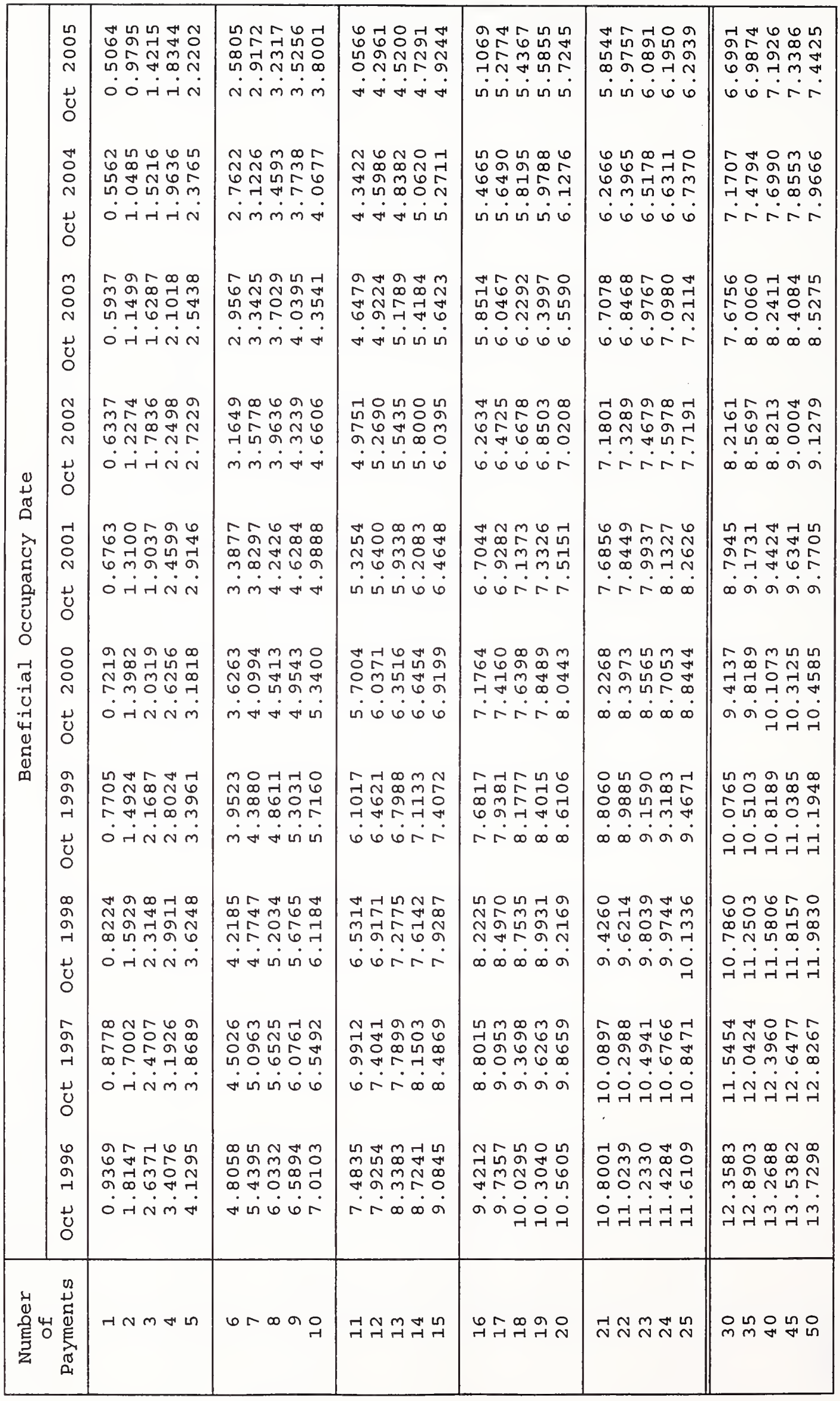

के न थत

तूर व

คำ 可

吸

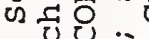

द् 40 .0

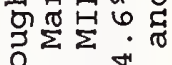

进类

मा पो 20

约质论

ने

I द

山昏。

U.

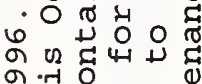
न- $\begin{array}{r}-1 \\ 0\end{array}$ 누 0 \%

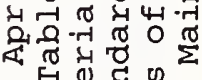

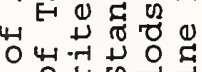
० 可武望

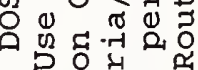
口0

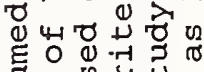
का o. 0 व

《斥 东

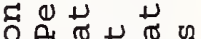

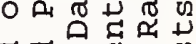

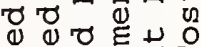

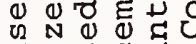
๙ ๓ म्रत ๙ 고권

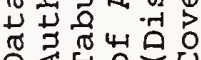
$\hat{i} \hat{v} \quad \hat{v}$ 


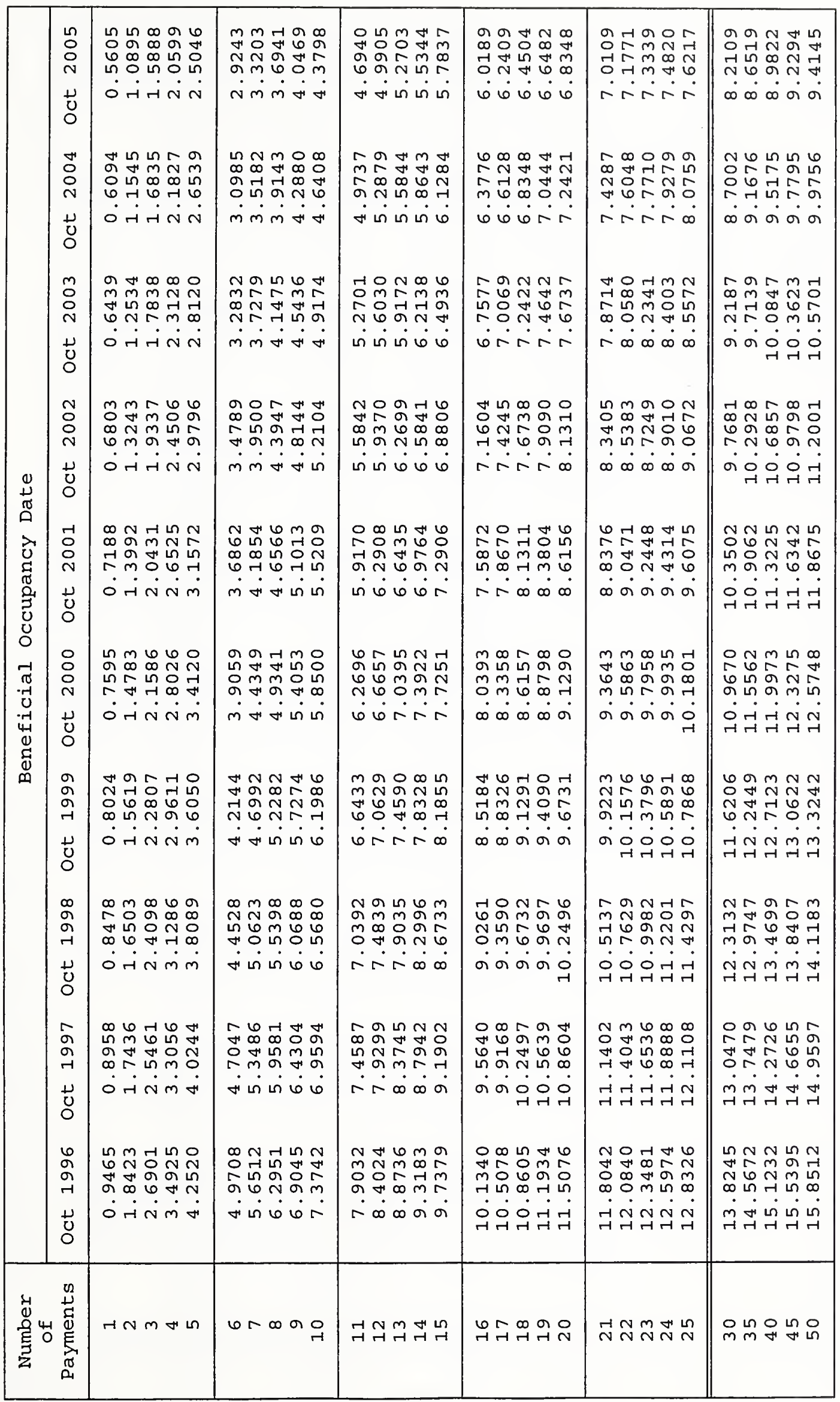


$6 m \sim \#+$

ㄴ. เก

Ninำ

rar

म

0तरNं

or

6 Nam

A $4 \sin 6$

O

gृ.

त्-

गु

叫

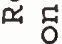

तif

तु

त्र

空

1

is $\pi$

मै

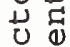

[1

ᄃ 4

남

30

प

要

(1) '

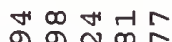

m च $\mathrm{m} \infty$

$\infty 6$ स त

HNG

ฝூ

क m 6 우 6

त $\infty \mathrm{m}$ \%

ornNm

अ

$6 \sim r \infty r$ लำ N 0 เก 0 न

숭요 的的 rnatgo

당

tom $\infty$ $\sim \sim \infty \infty \infty \dot{\infty}$

60060 क人 बे के mरत बं बं 000

Hman

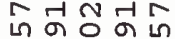
न $\sigma$ क $0 \sim m \sim ⿻ 上 丨$ mब6नr $m \infty m \infty \sim$ स स In 66 त $\infty \dot{\sigma}$

4 เึ

든 我组

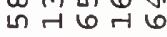
०तNM 舟

a mbrim A แ

$\therefore \infty \dot{\infty} \dot{\sigma}$

की

으무

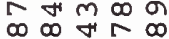

의 $\begin{array}{lll}0 \\ \infty & \infty & \pi\end{array}$

$\infty$ n

- m० $6 \mathrm{~N}$

H. 0 HNm

अ 10 6

$\infty$ न 丿 n⿺

U

の m

웅

$6 \mathrm{Nm}$ L $N$ त $\infty \circ 0$

$\infty m \omega\llcorner m$ ํำ $\therefore$ तो $0 \mathrm{~m}$

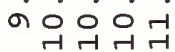

करत क

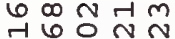
ㄴํำ 암닥다

-10 닥요 $\mathrm{N}$ ตी $\mathrm{N} 6$ NNNMm नतनमें

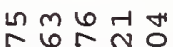
กำ

$m-1 n \circ N$ m年 เก

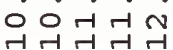
$60 \forall \infty$ vंmmं $4{ }^{4}$
0

4.

$m-1 \infty-16$ m이요요

ने ดํำ $\infty \pi m \infty$ H $\therefore m_{\infty}^{\infty}$ नू०

$0 \infty r+r$

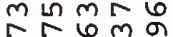

$\stackrel{1}{0}$

$\stackrel{1}{\sim}$

且

롱

-

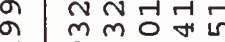

त बळरा

ग 0 तind

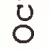

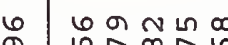

L

ส้の ๙

+ 166

$\infty \infty \sigma^{\circ} \dot{0}$

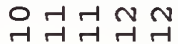

$m$ ต $6 m$ in अ 9 क

$\left.\begin{array}{r}m \\ -1 \\ -\infty\end{array}\right)$

Nm乚r

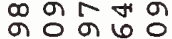

*

a mant ํํำ

$m \stackrel{m}{m} \stackrel{N}{\sim}$

แ

$\infty$ बं बे०

Нન

$m a$ เก $N$ กิ่ เึ็

mor

6 둥ํㄴ

U 0 तं

$\infty$ A $0, \pi$

4⿻上丨

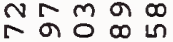
कब

ती

m $m \dot{m} \dot{\nabla}$

तथ

ropint

다옹ำ

6

$m \forall \nleftarrow 4 \operatorname{rn}$

HAत

은

अ 0 in $\mathrm{m}$

तิ

H

स 4 in $\ln$ in

क당ำ $\infty$ Nं。 mon 위유

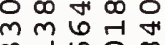
ตे $6 \infty$ a $0 \pi$ $m m m m b$ ำ

or $\infty$ कां

तथ

$+$

岂出

-

$+$

\begin{tabular}{|c|c|c|c|}
\hline 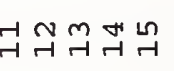 & 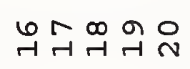 & $\stackrel{r}{\sim} \sim \stackrel{m}{\sim} \stackrel{\sim}{\sim}$ & 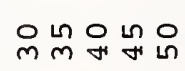 \\
\hline
\end{tabular}

I $m$ 깅

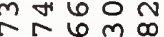

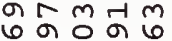

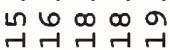
H $\rightarrow$ r 


\begin{tabular}{|c|c|c|c|c|c|c|c|}
\hline \multirow{10}{*}{ 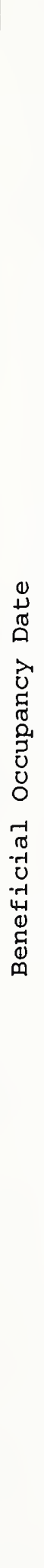 } & $\begin{array}{l}10 \\
0 \\
\circ \\
N \\
\dot{\nu} \\
0 \\
0\end{array}$ & 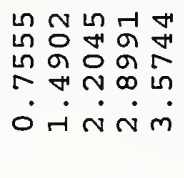 & 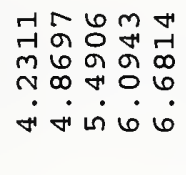 & 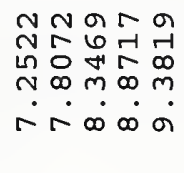 & 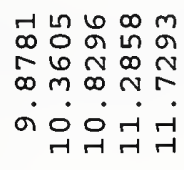 & 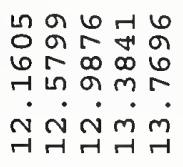 & 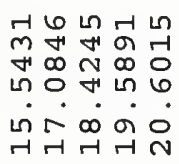 \\
\hline & 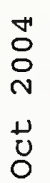 & 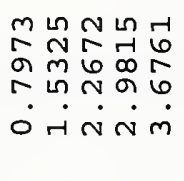 & 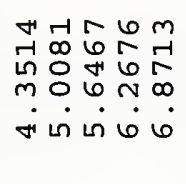 & 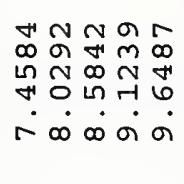 & 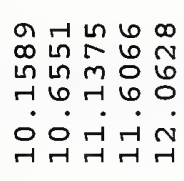 & 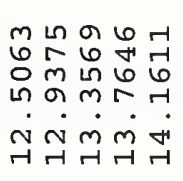 & 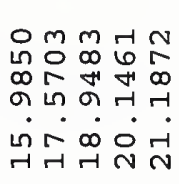 \\
\hline & 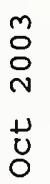 & 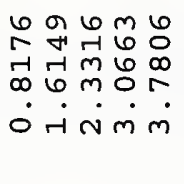 & 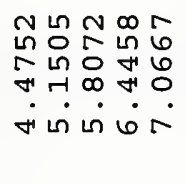 & 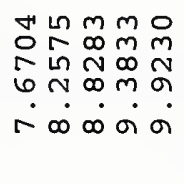 & 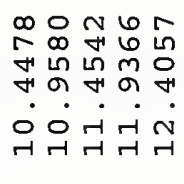 & 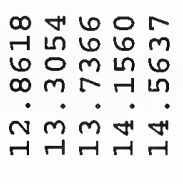 & 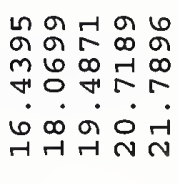 \\
\hline & 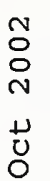 & 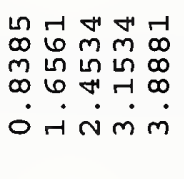 & 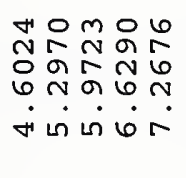 & 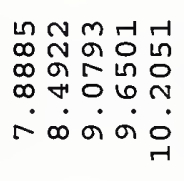 & 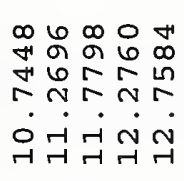 & 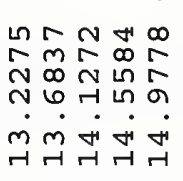 & 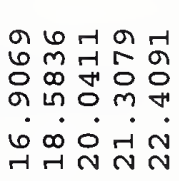 \\
\hline & 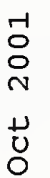 & 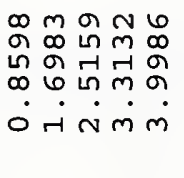 & 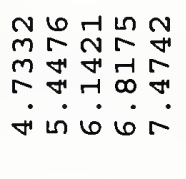 & 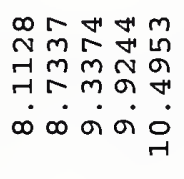 & 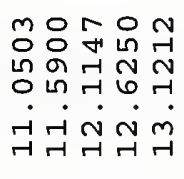 & 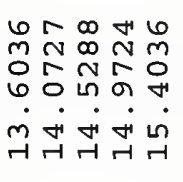 & 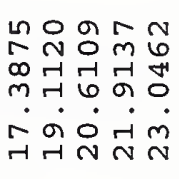 \\
\hline & $\begin{array}{l}\circ \\
\circ \\
\circ \\
\text { N } \\
\bigsqcup \\
0 \\
0\end{array}$ & 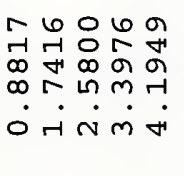 & 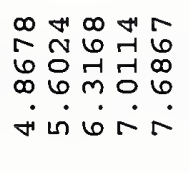 & 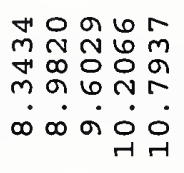 & 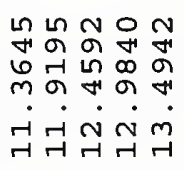 & 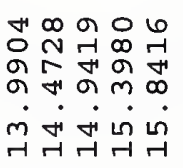 & 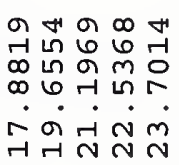 \\
\hline & $\begin{array}{l}\sigma \\
\sigma \\
\sigma \\
-1 \\
\overrightarrow{0} \\
0\end{array}$ & 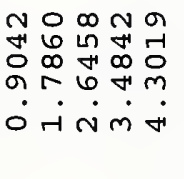 & 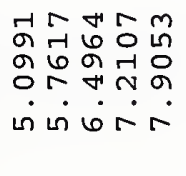 & 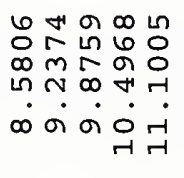 & 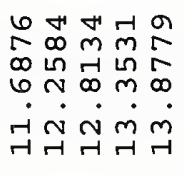 & 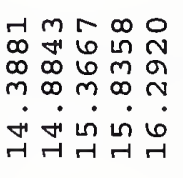 & 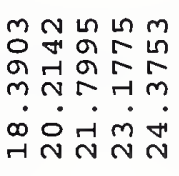 \\
\hline & $\begin{array}{l}\infty \\
\infty \\
\sigma \\
-1 \\
4 \\
0 \\
0\end{array}$ & 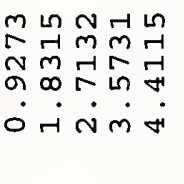 & 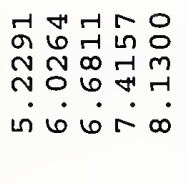 & 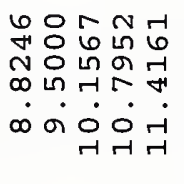 & 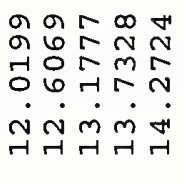 & 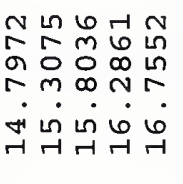 & 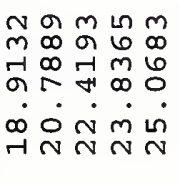 \\
\hline & 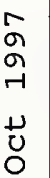 & 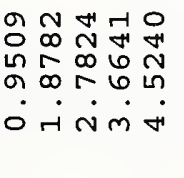 & 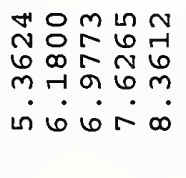 & 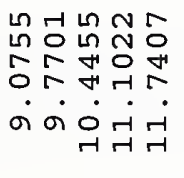 & 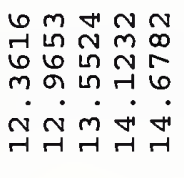 & 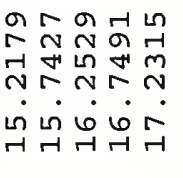 & 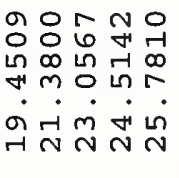 \\
\hline & $\begin{array}{l}6 \\
2 \\
2 \\
-1 \\
4 \\
0 \\
0\end{array}$ & 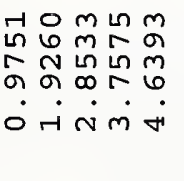 & 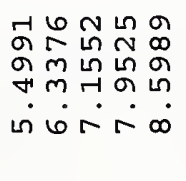 & 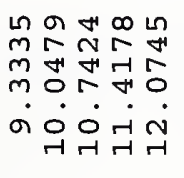 & 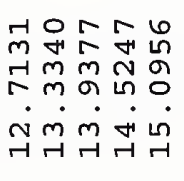 & 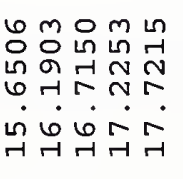 & 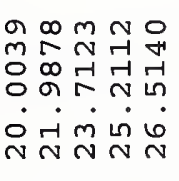 \\
\hline 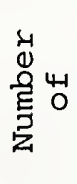 & $\underset{0}{n}$ & HNM $\mathrm{m}$ & $6 \pi \infty \sigma \underset{r}{0}$ & 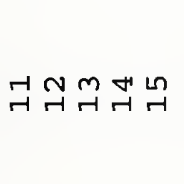 & 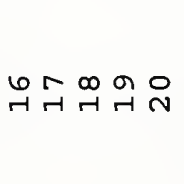 & $\stackrel{-}{\sim} \stackrel{m}{\sim} \stackrel{\sim}{\sim} \stackrel{n}{N}$ & 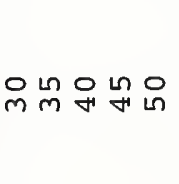 \\
\hline
\end{tabular}

NNano 에

HNN กN $N$ $\pi \infty$

tin m mo $-\operatorname{N} \infty \mathrm{N}$ - $\infty \sigma a$

In $\mathrm{Nm}-1$ $\infty$ बर स० - $\infty$ बन

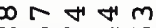
mmNo -.? + $\infty 000$ mbNr $\infty \infty \pi \circ 0$

6 स $\infty \mathrm{m}$ \% ก $\infty \pi$

o O N ก의 $\infty$ L क 000 -

ก int का H (ब

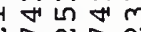
的 त 


\begin{tabular}{|c|c|c|c|c|c|c|c|}
\hline & 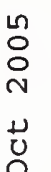 & 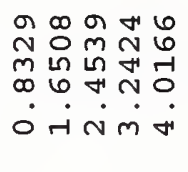 & 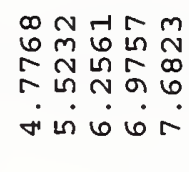 & 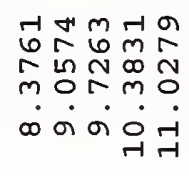 & 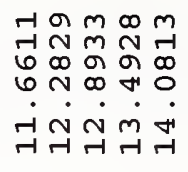 & 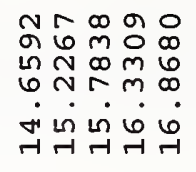 & 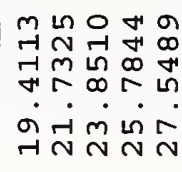 \\
\hline & $\begin{array}{l}\vec{J} \\
0 \\
0 \\
N \\
\stackrel{u}{0} \\
0\end{array}$ & 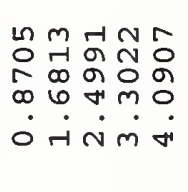 & 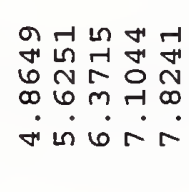 & 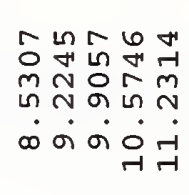 & 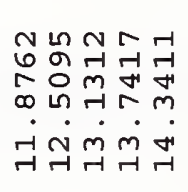 & 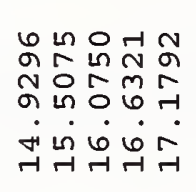 & 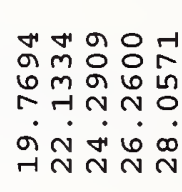 \\
\hline & $\begin{array}{l}m \\
o \\
O \\
\text { N } \\
\nu\end{array}$ & 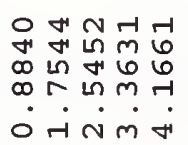 & 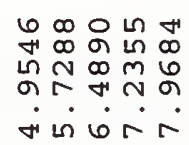 & 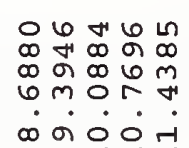 & 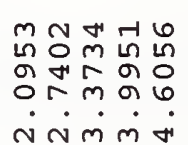 & 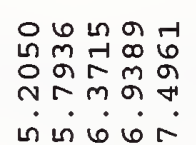 & 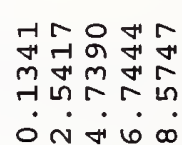 \\
\hline & 0 & & & (7 & 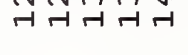 & 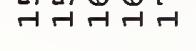 & $\sim \sim \sim \sim N$ \\
\hline & $\begin{array}{l}\text { N } \\
\text { O } \\
\text { N }\end{array}$ & 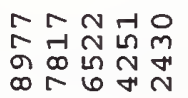 & 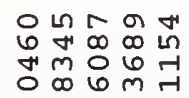 & 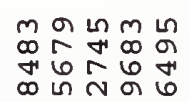 & 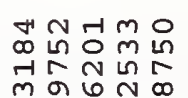 & 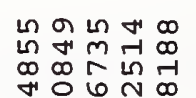 & 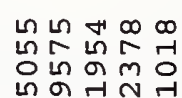 \\
\hline 0 & $\overleftrightarrow{u}$ & $\dot{\circ} \dot{\sim} \dot{m} \dot{r}$ & in in 6 & $\infty \sigma^{\circ} 0 \dot{-}$ & 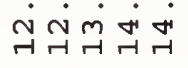 & in & 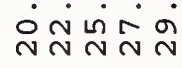 \\
\hline 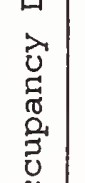 & $\begin{array}{l}-1 \\
0 \\
0 \\
\text { v } \\
\Delta \\
0 \\
0\end{array}$ & 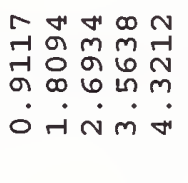 & 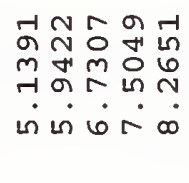 & 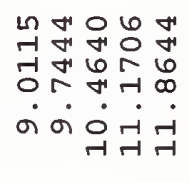 & 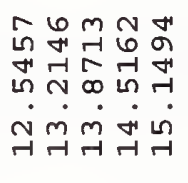 & 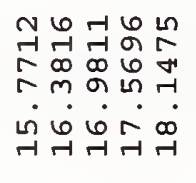 & 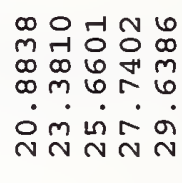 \\
\hline $\begin{array}{l}-1 \\
-1 \\
-1 \\
-1 \\
-1\end{array}$ & $\begin{array}{l}\text { O } \\
\text { O } \\
\text { N } \\
\text { U } \\
0 \\
0\end{array}$ & 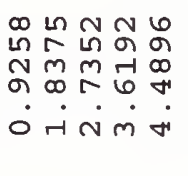 & 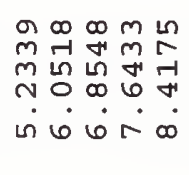 & 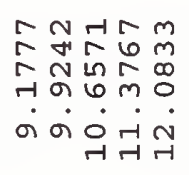 & 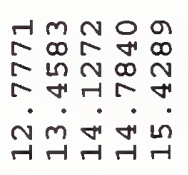 & 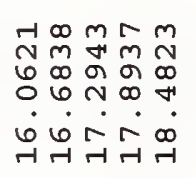 & 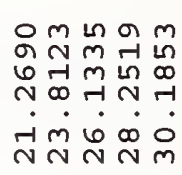 \\
\hline m & $\begin{array}{l}\sigma \\
\sigma \\
\sigma \\
\text { r } \\
u \\
0\end{array}$ & 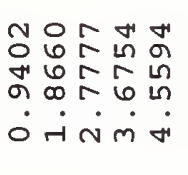 & 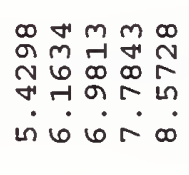 & 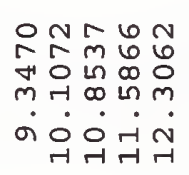 & 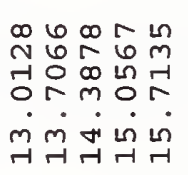 & 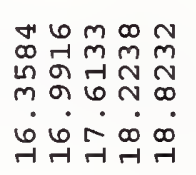 & 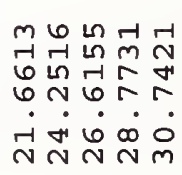 \\
\hline & $\begin{array}{l}\infty \\
\infty \\
\sigma \\
\text { - }\end{array}$ & 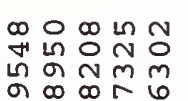 & 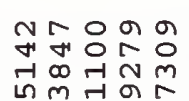 & 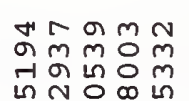 & 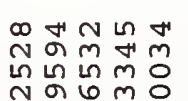 & 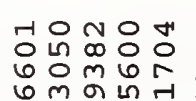 & 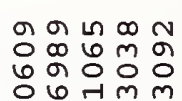 \\
\hline & Uू & $\dot{\circ} \dot{\sim} \dot{\mathrm{N}} \dot{\mathrm{m}}$ & $\dot{\varphi} \dot{0} \dot{\sim}$ & 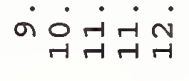 & 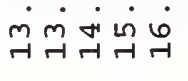 & $\begin{array}{l}\dot{0} \\
\vec{H}\end{array}$ & $\stackrel{\sim}{\sim} \stackrel{\sim}{\sim} \stackrel{\sim}{\sim} \stackrel{-}{m}$ \\
\hline & $\begin{array}{l}\hat{\sigma} \\
\text { ò } \\
\text { r }\end{array}$ & 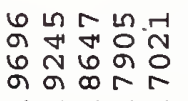 & 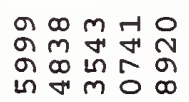 & 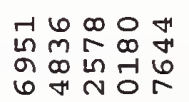 & 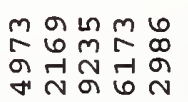 & 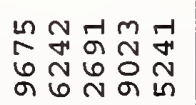 & 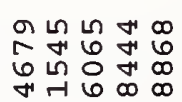 \\
\hline & $\stackrel{u}{0}$ & $\dot{\circ}$ - & $\dot{\omega} \dot{0} \sim \infty$ & 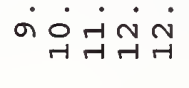 & 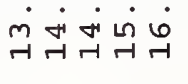 & 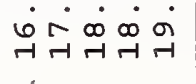 & $\stackrel{\sim}{\sim} \stackrel{\sim}{\sim} \stackrel{\sim}{\sim} \stackrel{-}{\sim}$ \\
\hline & $\begin{array}{l}6 \\
\text { } \\
\text { a } \\
-1\end{array}$ & 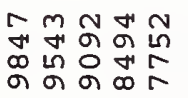 & 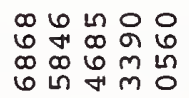 & 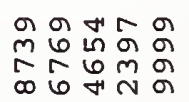 & 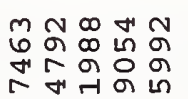 & 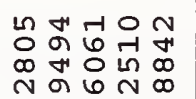 & 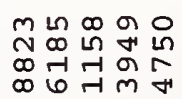 \\
\hline & $\stackrel{U}{0}$ & 0 HN & in $\dot{0} \infty$ & 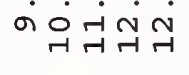 & 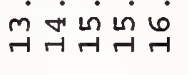 & 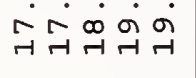 & $\dot{\sim} \stackrel{i}{\sim} \stackrel{\infty}{\sim} \dot{\sim} \dot{m}$ \\
\hline 嵌 & 点 & નNM & 6 & 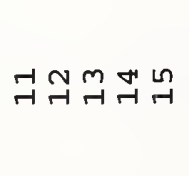 & $\begin{array}{l}0 \\
\text { બન }\end{array}$ & $\stackrel{-1}{N} \underset{N}{\sim} \stackrel{\sim}{N} \stackrel{\sim}{\sim}$ & 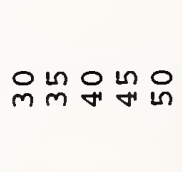 \\
\hline
\end{tabular}

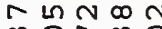

ก经的 药 ก $\mathrm{N}$ ब เ $\mathrm{N}$ कबन०

\% 6 \% 6 $\infty$ o $\infty 6 \mathrm{~m}$ m m N $-1 m o$ N $N$ Un 


\begin{tabular}{|c|c|c|c|c|c|c|c|}
\hline \multirow{10}{*}{ 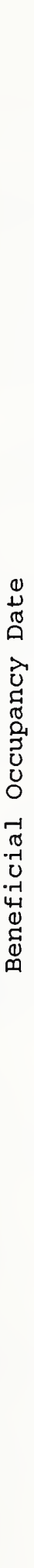 } & $\begin{array}{l}\text { Ln } \\
\text { O } \\
\stackrel{0}{N} \\
1 \\
\text { U } \\
0\end{array}$ & 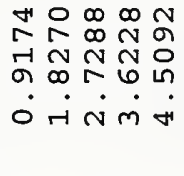 & 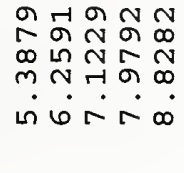 & 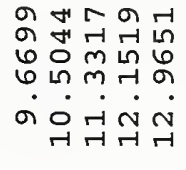 & 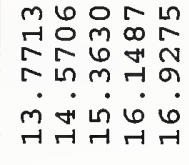 & 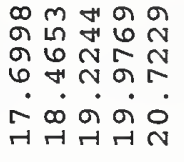 & 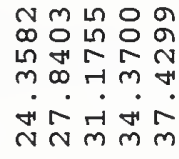 \\
\hline & 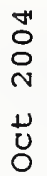 & 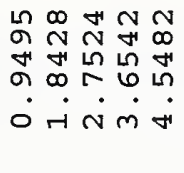 & 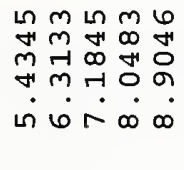 & 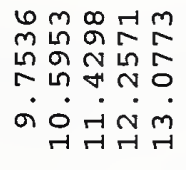 & 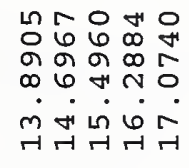 & 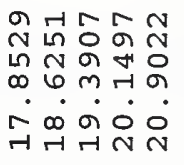 & 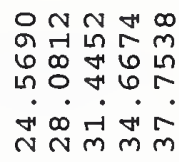 \\
\hline & $\begin{array}{l}m \\
\circ \\
\circ \\
N \\
1 \\
U \\
0\end{array}$ & 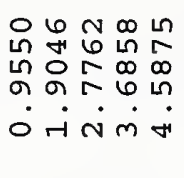 & 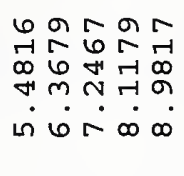 & 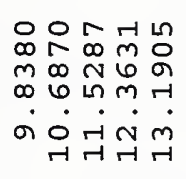 & 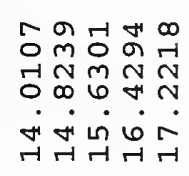 & 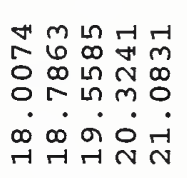 & 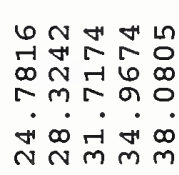 \\
\hline & 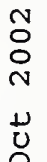 & 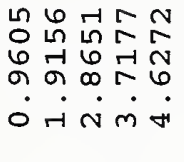 & 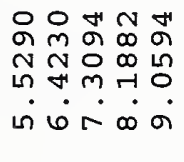 & 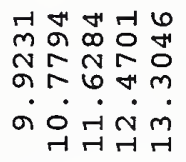 & 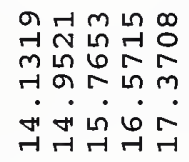 & 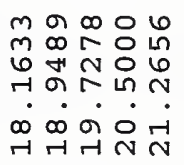 & 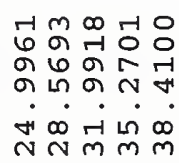 \\
\hline & $\begin{array}{l}-1 \\
O \\
O \\
\text { N } \\
1 \\
0 \\
0\end{array}$ & 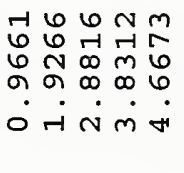 & 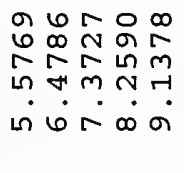 & 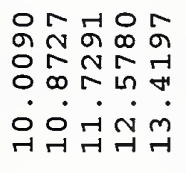 & 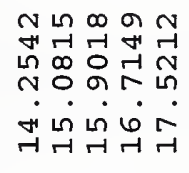 & 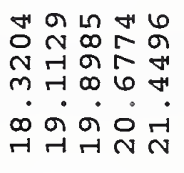 & 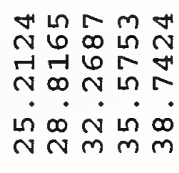 \\
\hline & $\begin{array}{l}O \\
\circ \\
\circ \\
\sim \\
1 \\
0 \\
O\end{array}$ & 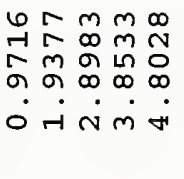 & 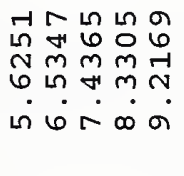 & 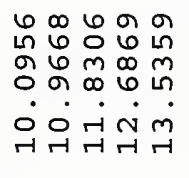 & 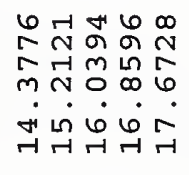 & 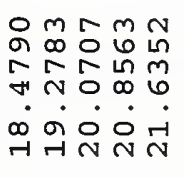 & 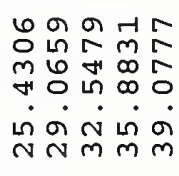 \\
\hline & $\begin{array}{l}\text { न } \\
\text { ने } \\
\text { ने } \\
+ \\
0 \\
0\end{array}$ & 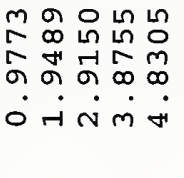 & 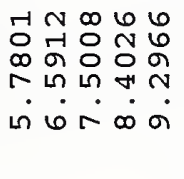 & 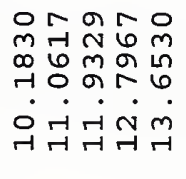 & 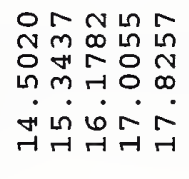 & 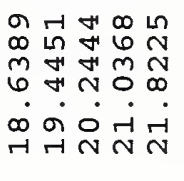 & 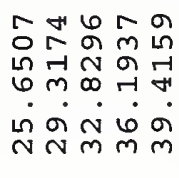 \\
\hline & $\begin{array}{l}\infty \\
\sigma \\
\sigma \\
ન \\
\vdots \\
0 \\
0\end{array}$ & 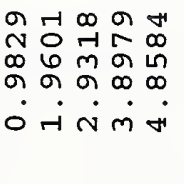 & 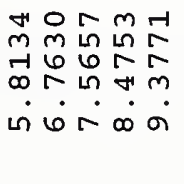 & 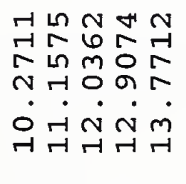 & 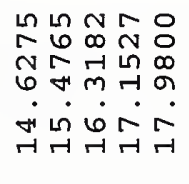 & 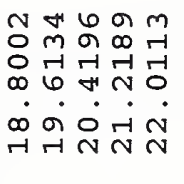 & 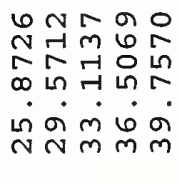 \\
\hline & $\begin{array}{l}\hat{\sigma} \\
\sigma \\
\sigma \\
r 1 \\
1 \\
0 \\
0\end{array}$ & 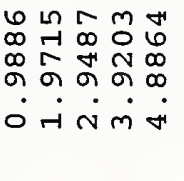 & 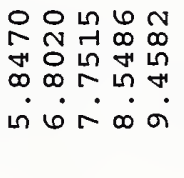 & 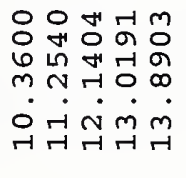 & 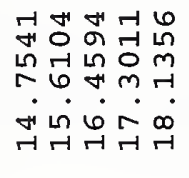 & 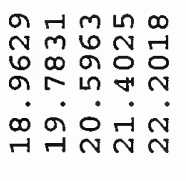 & 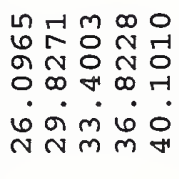 \\
\hline & $\begin{array}{l}6 \\
\sigma \\
\sigma \\
न 1 \\
1 \\
0 \\
0\end{array}$ & 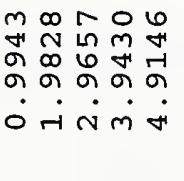 & 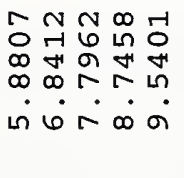 & 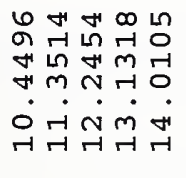 & 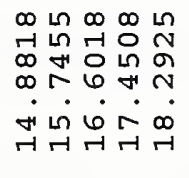 & 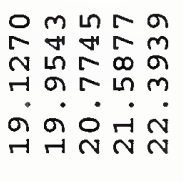 & 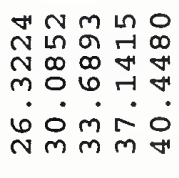 \\
\hline 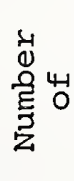 & $\sum_{\substack{0 \\
0}}^{+}$ & HNM & br $\infty \underset{-1}{0}$ & ન્ન & 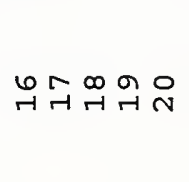 & $\stackrel{\sim}{N} \stackrel{N}{N} \stackrel{\sim}{N} \stackrel{\sim}{N}$ & 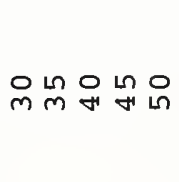 \\
\hline
\end{tabular}




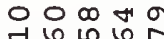

늄뵤

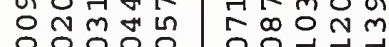

○००o

तN

0

。े

an n

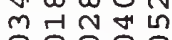

०००00

HNM

$m \in \tilde{m}$

00000

HNm

空

त्र

प्रे 11

บ 1

幽

$>$ 号

न1

(2)

究

嵒

in -

กุ

돈

U

江

도 4

농

3

$\pm$

过

is 1

¿

虹

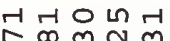

ก เ

1

HNM

mor 60

HNm

man

강

HNm n

*1 $\infty$ a

ป

$0000 \mathrm{~T}$

แก 드

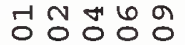

1

1

穵

峁

ए厂

म $6 \%$ 다 तन त N

- $\operatorname{con} \mathrm{N}$ $6 \infty \pi+$ 西 or $\infty 0$

$m \infty \sim \pi$ $6 \stackrel{\infty}{r} 0 \mathrm{~m}$ o 0 H 6N 00

60 ก ती 00004 bN 00

$\infty m \infty \sim$ $\infty \circ \omega 60$ जिक

은 m 00000 ur

$\infty a \pi$ 年 - 0000

A A ก त 1000 कर

Uั

- $\sim N 0_{0}$ ๙ r 00000

더겅의 6 응은 तn जर $\infty 0$

$\infty$ n m그 엉잉

कののの 6 क $50 m$ m क으요 மم $\infty \dot{0}$ กNm

艺

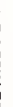
4
$4 \pi$

- $160 \mathrm{~m}$

단됴
N N N

ㄴํㅇㅇㅛ

각잉

ก

तn

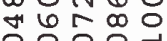
மா नr त

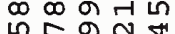
HNm

N m 융유 든 N $\mathrm{mm}$ 6r $\infty 0$

$\infty \circ N+6$ * 6000 Hก HNm

n n กับ तन तम

几 납 न

o $m \omega n$

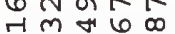
तन HNm नतम

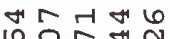
पूत तन न

$\infty N$ เ

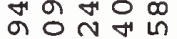
0 तन तु न $N m$ a तथ

NeO ar कू के กิ ठ० m O००न 대 $m$



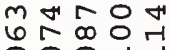
$000 \mathrm{O}$ न

$\sim \infty \sigma a \circ$ 经 N $\mathrm{m} m \mathrm{~m}$

$\mathrm{N} m$ เก เก 6

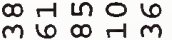
กำ $\stackrel{\infty}{m} m$ 6r $\infty \circ$ न

- $20 \mathrm{~N}$ N N N N or $\infty 0$ न

$m \cos a$ 용요 ก ก ก wr $\infty$ a 0 न

बतm

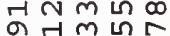

or $\infty$ a 0 H H

4र 는 Н $\mathrm{r} N$

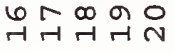

- $m$ un $\infty$ 궁 तन त जत

6ด เ $\forall \begin{aligned} & N \\ & \forall \\ & 0\end{aligned}$ Hก 두요

m69

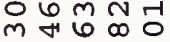
न4त $6 \pi \infty$ o 0 नतन त नNm HNm तNm HNm $m$ or $\infty \circ$ ก ก ก

西 $m$

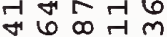
N N $\mathrm{m} m$ ㄱN $\stackrel{m}{\sim} \stackrel{\sim}{N}$

4 2605 $\infty \circ m$ in 政 궈ํํำ

- $N m$ m $6 \infty 0 \mathrm{~m}$ . ㄷN표

\section{no $m m m$} ก 눙 있 누의

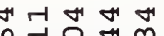

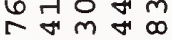
$\rightarrow 6 \infty 0 \stackrel{\infty}{\circ}$

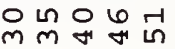
กั ง $\sim$ 게 $m$ U NNNN

$\Delta 4 \ln 6$ न 0 न잉 4 ำ

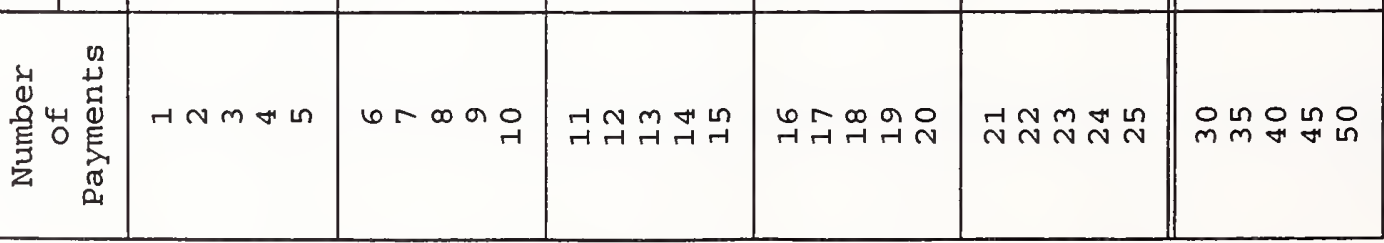

ᄃ 4 거 do 引 나 \& y म 氙秀 几 बन्न ट ठै न

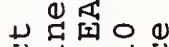
U - $\bigcirc$ 告 ดे थ

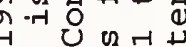
(थ) 包

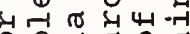

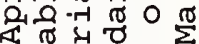
स 山山

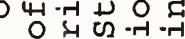
य 0 क्ष

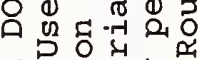
ס

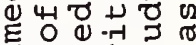
E 0 ब 证牙记 on 乐 $\sigma$ Oٓ

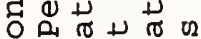
व ช U N Q ๓

O

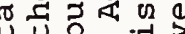

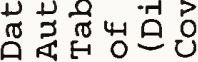
$\hat{v} \hat{v} \quad \hat{v}$ $\ddot{0}$
0
0
0 


\section{Appendix A}

\section{MEMORANDUM OF AGREEMENT ON CRITERIA/STANDARDS FOR ECONOMIC ANALYSES/LIFE CYCLE COSTING FOR MILCON DESIGN, MARCH 1994}

1. Purpose. The purpose of this Memorandum of Agreement (MOA) is to establish criteria and standards for performing economic analyses and life cycle cost studies used in support of design decisions for projects in the Military Construction (MILCON) Program, i.e., to support the selection from various alternatives of components/systems being considered as elements in facilities design. These criteria and standards apply to all design decisions regardless of when they are made in the overall facilities acquisition process. This agreement does not apply to economic analyses and life cycle cost studies used to make project-justification decisions during that process.

2. General. Economic analyses shall be conducted as part of the design process to ensure that the selection/rejection of design alternatives is not based solely on construction costs, but also on least life cycle costs (LCC), that is, lowest total cost of ownership. The depth and degree of formality of these analyses shall be determined on a case-by-case basis to ensure that the cost of performing an analysis is clearly outweighed by the potential benefits derived. Results of generic studies or results of previous analyses of alternatives similar to those currently under consideration may be used in lieu of performing a new study, provided (a) the previous study was based on similar design conditions, criteria, and methods, and (b) the results of the previous study appear valid and applicable for the current project. Previous studies should be updated only as required to reflect changes of conditions and/or errors significant enough to impact the design decision. All economic analyses and other justification for the selection of a design alternative, whether a previous study or a new one, shall be clearly documented in the appropriate section of the project design analysis.

3. Methods. All analyses shall consider the total LCC for design alternatives, where the LCC includes all costs and benefits associated with an alternative over its expected life, including but not limited to construction/acquisition, energy, maintenance, operation, repair, replacement, alteration, disposal costs, and retention values. The present value discounting approach shall be used to adjust for the difference in timing of costs and benefits unless otherwise specified by other directives or by public law. The basic discount factor for finding the present value of a future amount is calculated as follows:

$$
\text { discount factor }=\frac{1}{(1+d)^{n}}
$$

where: $\quad \mathrm{d}=$ appropriate discount rate, and

$\mathrm{n}=$ the time period over which the discounting is done.

Discounting should be applied to all costs and benefits over the appropriate analysis period. Specific criteria are as follows:

a. Discount Rates. The discount rates are expressed in "real" terms, i.e., over-and-above the rate of inflation for the economy as a whole.

(1) Energy related studies: All energy related economic studies (studies in which energy costs are relevant, regardless of their magnitude relative to other costs) shall use the current discount rate published by the National Institute of Standards and Technology (NIST) in their annual supplement to NIST Handbook 135, and disseminated by the appropriate Service Headquarters Office. 
(2) Non-energy related studies: All economic studies other than energy related economic studies shall use the current discount rates published annually by the Office of Management and Budget (OMB) as APPENDIX C to OMB CIRCULAR NO. A-94, and disseminated by the appropriate Service Headquarters Office.

b. Analysis Period. The analysis period shall be the date of the study (DOS) through the economic life of the facility as a whole. The economic life shall not be taken beyond 25 years from the scheduled beneficial occupancy date (BOD) for the project unless specifically approved by the appropriate Service Headquarters Office. Such approval cannot be granted for energy related studies as it is precluded by statute.

c. Cash Flow. In general, cash flow used in the analysis will be based on the estimated calendar dates on which the events and costs/benefits are projected/scheduled to occur. Construction/acquisition costs may be assumed to be incurred as a single lump sum, preferably at the time corresponding to the midpoint of the construction/acquisition process. Other cash flows that occur periodically throughout the year (e.g., cost of fuel, electricity, water, maintenance, etc.) may be assumed to be incurred as a single lump sum, preferably at midyear. In circumstances where the above assumptions add unnecessarily to the complexity of the calculations, all cash flows may be assumed to occur at the end of the year in which they are actually scheduled/projected to occur.

d. Benefits and Costs. All benefits and costs will be expressed in terms of constant dollars that reflect the purchasing power of the dollar on the DOS (i.e., constant DOS dollars). The rate of inflation of the economy as a whole will be excluded from all LCC calculations. (The rate of inflation is irrelevant to the LCC analysis results since all benefits and costs are expressed in terms of constant DOS dollars and discounted using a "real" discount rate which reflects the time value of money over-and-above the general rate of inflation.)

e. Future Benefits and Costs. In projecting future benefits and costs, an allowance for future pricelevel changes will be made only for particular benefits and costs expected to change at rates higher or lower than the general rate of inflation. In such cases, the rates of change used in the analysis will be the "differential" rates, i.e., the anticipated differences between the actual projected rates of change and the general inflation rate.

(1) Energy studies: Fuel/energy costs shall have differential escalation rates as published by NIST in Handbook 135 and disseminated as indicated in paragraph 3.a(1) above. All nonenergy costs shall have a zero differential escalation rate.

(2) Non-energy studies: For non-energy studies, the differential rate of future price-level change shall generally be assumed to be zero, except in those cases where there is reliable information/data to the contrary.

(4) Computer Aided Calculations. All computer aided calculations for MILCON design economic studies will be accomplished using the Life Cycle Cost in Design (LCCID) computer program for economic analysis developed by the U.S. Army Corps of Engineers Construction Engineering Research Laboratory (CERL) or a version thereof which has been certified by CERL as equivalent. 

